\title{
Indolizines and Their Hetero/Benzo Derivatives in Reactions of [8+2] Cycloaddition
}

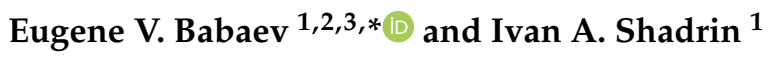 \\ 1 Chemistry Department, Moscow State University, Leninskie Gory, 1 Str. 3, 119899 Moscow, Russia; \\ i.shadrin666@gmail.com \\ 2 Higher School of Economics, National Research University, 7 Vavilova Str., 117312 Moscow, Russia \\ 3 N. D. Zelinsky Institute of Organic Chemistry, Russian Academy of Sciences, 47 Leninsky Ave., \\ 119991 Moscow, Russia \\ * Correspondence: babaev@org.chem.msu.ru; Tel.: +7-985-997-94-75
}

check for

updates

Citation: Babaev, E.V.; Shadrin, I.A Indolizines and Their Hetero/Benzo Derivatives in Reactions of [8+2] Cycloaddition. Molecules 2021, 26, 2050. https://doi.org/10.3390/ molecules 26072050

Academic Editor: Ionel Mangalagiu

Received: 17 March 2021

Accepted: 30 March 2021

Published: 3 April 2021

Publisher's Note: MDPI stays neutral with regard to jurisdictional claims in published maps and institutional affiliations.

Copyright: (c) 2021 by the authors. Licensee MDPI, Basel, Switzerland. This article is an open access article distributed under the terms and conditions of the Creative Commons Attribution (CC BY) license (https:// creativecommons.org/licenses/by/ $4.0 /)$.
Abstract: Peculiarities of [8+2] cycloaddition of acetylenes to indolizines are reviewed. Especially mentioned are indolizines with leaving groups at positions 3 and 5. Cycloaddition to aza- and benzo derivatives are reviewed, as well as 1,10-cyclizations and processes leading to cyclazines where indolizines are intermediates. Mechanistic features (adducts and cycloadducts) and theoretical aspects (one- or two-steps mechanism) are reviewed.

Keywords: indolizine; azaindolizines; benzoindolizines; cyclazine; [8+2] cycloaddition; mechanism; 1,10-cyclizations; catalysts

\section{Introduction}

Indolizine (A, Scheme 1$)$ is the simplest heteroaromatic molecule containing both a $\pi$-excessive pyrrole and a $\pi$-deficient pyridine ring with only one bridgehead nitrogen, the whole system being isomeric with indole and possess pharmaceutical, agrochemical and fluorescent properties [1]. Although indolizine is certainly aromatic, significant alternations of the bond lengths around the ring system were detected by X-ray, NMR and UV spectroscopy and even mass spectrometry in various substituted indolizines. This prompts some tetraene-like character of the compound, in particular its ability to enter into cycloaddition reactions.

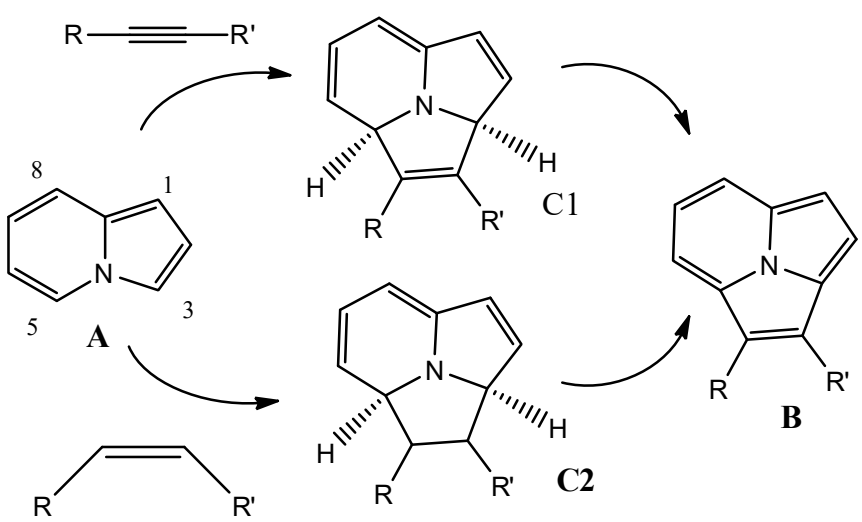

Scheme 1. Indolizines (A) in [8+2] cycloaddition reaction forming cycl[3.2.2]azines (B). The reaction may proceed with alkynes or alkenes via dihydro- (C1) or tetrahydro- (C2) cyclazines.

Indolizine is usually regarded as the $\pi$-excessive heterocycle with the highest electron population of the carbon atom $\mathrm{C}-3$, and the major part of the chemistry of indolizines 
is simple electrophilic addition and substitution at this position. Cycloaddition of various dienophiles (alkenes and acetylenes) to indolizines leading to derivatives of the cycl[3.2.2]azine (B, Scheme 1) is well-known. The mechanism of these reactions is frequently regarded as a rare example of [8+2] cycloaddition, where the tetraene carbon framework of the indolizine bicycle plays the role of an $8 \pi$-electron fragment. In general, this process may be either one-step (concerted) or involve zwitterionic (and even biradical) intermediates, and there is yet no experimental evidence for the nature of the process.

Cyclazine (B) is an interesting $12 \pi$-electronic system that breaks the canons of aromaticity. According to X-ray data the structures B1 and B2 are not correct (Scheme 2), and the structure rather has a peripheral delocalization of aromatic $10 \pi$-electron system B3. Therefore, cyclazine resembles the famous spinning toy $\mathbf{B} 4$ where the handle (which is not rotated) corresponds to nitrogen lone pair. Hence, the structure has a symmetry plane, and this influences the number of positional isomers, say the number of aza- and benzo-derivatives possible for cyclazine (Scheme 3).

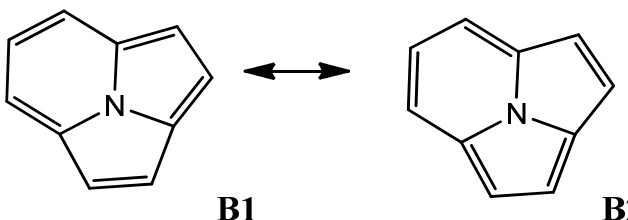

B2<smiles>c1cc2ccc3ccc(c1)n23</smiles>

B3

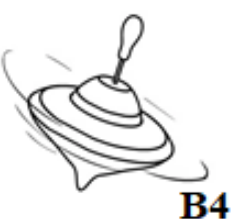

Scheme 2. Different images of the structure of cyclazine.

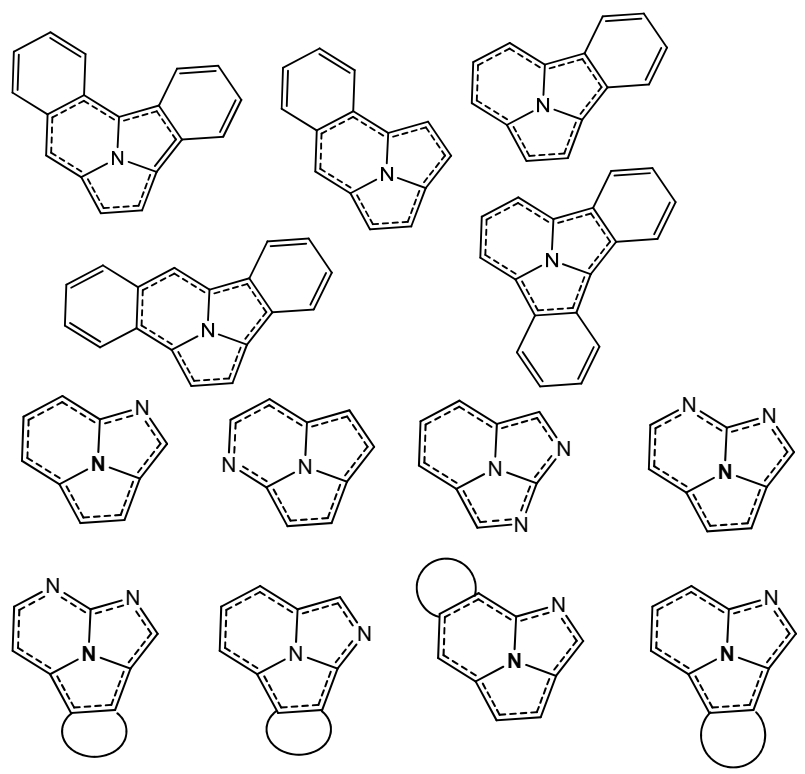

Scheme 3. Possible benzo- aza- and benzoazacyclazines discussed in this review.

Cyclazins and their hetero/benzo derivatives are important from a practical viewpoint. They are fluorescent compounds and have excellent prospects in organic electronics [2-8]. On the other hand, biological activity was found in cyclazines, and their applications as estrogens and anti-inflammatory compounds are well known [9-11].

Cyclazine was first obtained from indolizine by Boekelheide 60 years ago. This author was the first who postulated the [8+2] mechanism. After this time the [8+2] reaction was reviewed several times. The first review by Acheson appeared in 1963 [12] and the next one by Taurins in 1977 [13]. Several reviews were written on the chemistry of cyclazines [1,14-19]. In the reviews of Nair and Abhilash [20,21] devoted to [8+2] cycloaddition reactions, only a limited number of indolizine reactions was mentioned. 
Therefore, this review may be considered as the first and comprehensive review on the [8+2] cycloaddition reactions between aza/benzo indolizines and acetylenes leading to cyclazines.

\section{Cyclazines from Indolizines via [8+2] Catalytic Cycloaddition}

The first cycloaddition to indolizine (entry 1 in Table 1 ) was observed by Boekelheide in 1959 [22] (Scheme 4) and later to 2 [23] by using DMAD and heating in toluene in presence of Pd-C. Recently reaction of 3 was reported with $\mathrm{MnO}_{2}$ as oxidant [4]. Boekelheide was the first who made the reaction of 4 with non-symmetric alkyne [24] and performed cycloaddition with DMAD to 2-Ph-indolizine 5 [25]; this reaction was repeated recently with 8a,b [26]. 2-Methylindolizine 6 was involved in the reaction with DMAD in 1965 [27], and the same reaction was done for 8- $R$-indolizines $7 \mathbf{a}-\mathbf{c}$ [28].<smiles></smiles><smiles>[R]C#CC</smiles>

Scheme 4. Symthesis of cyclazines from indolizines (Table 1).

Indolizines 9a-c obtained by desulfurization of 2-MeS derivatives were converted to cyclazines [29]. First indolizine 10 substituted by functional groups was involved in cyclization with DMAD in 1974 [30]. Later, this methodology was used to construct cyclophanes by applying 2-MeS-3-CONH${ }_{2}$ substituted structures $\mathbf{1 1 a}, \mathbf{b}$ [31,32]. Later 2MeS-3-COOR derivatives were hydrolyzed and decarboxylated to 12a-c and converted to cyclazines [29]. A similar methodology was used to construct cyclazine from 2-MeS7- $\mathrm{NMe}_{2}$-indolizine $13 \mathbf{b}$ : desulfurization gave $7-\mathrm{NMe}_{2}$ derivative $13 \mathbf{a}$ and the addition of DMAD gave corresponding cyclazine [2].

Fluoro-substituted indolizines are seldom [33] but 1-fluoro derivatives 14a,b underwent cycloaddition in oxidative condition in presence of $\mathrm{Cu}(\mathrm{II})$ salts [34]. One more example to introduce functionality to cyclazine is catalytic cycloaddition of 6,8-diacyl indolizine 17 [35]. In our recent work, we proved that MAC could react with 2-t-Bu indolizine 18 giving cyclazine in open-air [36]. One featured reaction was cycloaddition of indolizine 19 with $\mathrm{Mes}_{2} \mathrm{~B}$-substituted acetylene [37]. 2-Styryl indolizine reacted with DMAD and MAC without a catalyst [38]. Big series of cyclazines (though without the yields) was synthesized and described as estrogens $[9,10]$.

Table 1. Substituents, reaction conditions and yields of reactions of indolizines with substituted acetylenes giving cyclazines (Scheme 4).

\begin{tabular}{|c|c|c|c|c|c|c|c|c|c|}
\hline $\mathbf{N}$ & $\mathbf{R} / \mathbf{R}^{\prime}$ & R1 & $\mathbf{R} 2$ & R6 & R7 & R8 & Cat/Solv/T $/$ Time & Yield \% & Ref. \\
\hline 1 & $\mathrm{E} / \mathrm{E}^{\mathrm{a}}$ & $\mathrm{H}$ & $\mathrm{H}$ & $\mathrm{H}$ & $\mathrm{H}$ & $\mathrm{H}$ & $\mathrm{Pd}-\mathrm{C} / \mathrm{MePh} / \Delta$ & $50-66$ & [22] \\
\hline 2 & $\mathrm{E} / \mathrm{E}$ & $\mathrm{H}$ & $\mathrm{H}$ & $\mathrm{H}$ & $\mathrm{H}$ & $\mathrm{H}$ & $\mathrm{Pd}-\mathrm{C} / \mathrm{MePh} / \Delta / 24 \mathrm{~h}$ & 68 & [23] \\
\hline 3 & $\mathrm{E} / \mathrm{E}$ & $\mathrm{H}$ & $\mathrm{H}$ & $\mathrm{H}$ & $\mathrm{H}$ & $\mathrm{H}$ & $\mathrm{MnO}_{2} / \mathrm{MePh} / \Delta / 16 \mathrm{~h}$ & 55 & [4] \\
\hline 4 & $\mathrm{E} / \mathrm{H}$ & $\mathrm{H}$ & $\mathrm{H}$ & $\mathrm{H}$ & $\mathrm{H}$ & $\mathrm{H}$ & $\mathrm{Pd}-\mathrm{C} / \mathrm{MePh} / \Delta / 24 \mathrm{~h}$ & 11 & [24] \\
\hline 5 & $\mathrm{E} / \mathrm{E}$ & $\mathrm{H}$ & $\mathrm{Ph}$ & $\mathrm{H}$ & $\mathrm{H}$ & $\mathrm{H}$ & $\mathrm{Pd}-\mathrm{C} / \mathrm{MePh} / \Delta / 20 \mathrm{~h}$ & 28 & [25] \\
\hline 6 & $\mathrm{E} / \mathrm{E}$ & $\mathrm{H}$ & $\mathrm{Me}$ & $\mathrm{H}$ & $\mathrm{H}$ & $\mathrm{H}$ & $\mathrm{Pd}-\mathrm{C} / \mathrm{MePh} / \Delta$ & 60 & [27] \\
\hline $7 a$ & $\mathrm{E} / \mathrm{E}$ & $\mathrm{H}$ & $\mathrm{H}$ & $\mathrm{H}$ & $\mathrm{H}$ & $\mathrm{Me}$ & $\mathrm{Pd}-\mathrm{C} / \mathrm{MePh} / \Delta / 1.5 \mathrm{~h}$ & 53 & [28] \\
\hline $7 \mathrm{~b}$ & $\mathrm{E} / \mathrm{E}$ & $\mathrm{H}$ & $\mathrm{H}$ & $\mathrm{H}$ & $\mathrm{H}$ & $\operatorname{Pr}$ & $\mathrm{Pd}-\mathrm{C} / \mathrm{MePh} / \Delta / 1.5 \mathrm{~h}$ & 53 & [28] \\
\hline $7 \mathrm{c}$ & $\mathrm{E} / \mathrm{E}$ & $\mathrm{H}$ & $\mathrm{H}$ & $\mathrm{H}$ & $\mathrm{H}$ & $\mathrm{Ph}$ & $\mathrm{Pd}-\mathrm{C} / \mathrm{MePh} / \Delta / 1.5 \mathrm{~h}$ & 53 & [28] \\
\hline $8 a$ & $\mathrm{E} / \mathrm{E}$ & $\mathrm{H}$ & $\mathrm{Ph}$ & $\mathrm{H}$ & $\mathrm{H}$ & $\mathrm{H}$ & $\mathrm{Pd}-\mathrm{C} / \mathrm{MePh} / \Delta$ & 39 & [26] \\
\hline $8 b$ & $\mathrm{E} / \mathrm{E}$ & $\mathrm{H}$ & $\mathrm{p}-\mathrm{tBuPh}$ & $\mathrm{H}$ & $\mathrm{H}$ & $\mathrm{H}$ & $\mathrm{Pd}-\mathrm{C} / \mathrm{MePh} / \Delta$ & 75 & [26] \\
\hline $9 a$ & $\mathrm{E} / \mathrm{E}$ & $\mathrm{H}$ & $\mathrm{H}$ & $\mathrm{H}$ & $\mathrm{H}$ & $\mathrm{H}$ & $\mathrm{Pd}-\mathrm{C} / \mathrm{MePh} / \Delta / 100 \mathrm{~h}$ & 33 & [29] \\
\hline $9 b$ & $\mathrm{E} / \mathrm{E}$ & $\mathrm{H}$ & $\mathrm{H}$ & $\mathrm{H}$ & $\mathrm{H}$ & $\mathrm{Me}$ & $\mathrm{Pd}-\mathrm{C} / \mathrm{MePh} / \mathrm{D} / 100 \mathrm{~h}$ & 34 & [29] \\
\hline
\end{tabular}


Table 1. Cont.

\begin{tabular}{|c|c|c|c|c|c|c|c|c|c|}
\hline $\mathbf{N}$ & $\mathbf{R} / \mathbf{R}^{\prime}$ & $\mathbf{R} 1$ & $\mathbf{R} 2$ & R6 & R7 & R8 & Cat/Solv/T ${ }^{\circ} /$ Time & Yield \% & Ref. \\
\hline $9 c$ & $\mathrm{E} / \mathrm{E}$ & $\mathrm{H}$ & $\mathrm{H}$ & $\mathrm{Me}$ & $\mathrm{H}$ & $\mathrm{Me}$ & $\mathrm{Pd}-\mathrm{C} / \mathrm{MePh} / \Delta / 100 \mathrm{~h}$ & 25 & [29] \\
\hline 10 & $\mathrm{E} / \mathrm{E}$ & $\mathrm{CONH}_{2}$ & MeS & $\mathrm{H}$ & $\mathrm{H}$ & $\mathrm{H}$ & $\mathrm{Pd}-\mathrm{C} / \mathrm{MePh} / \Delta / 24 \mathrm{~h}$ & 70 & [30] \\
\hline $11 a$ & $\mathrm{E} / \mathrm{H}$ & $\mathrm{CONH}_{2}$ & MeS & $\mathrm{H}$ & $\mathrm{H}$ & $\mathrm{H}$ & $\mathrm{Pd}-\mathrm{C} / \mathrm{MePh} / \Delta / 100 \mathrm{~h}$ & 45 & [31] \\
\hline $11 b$ & $\mathrm{E} / \mathrm{H}$ & $\mathrm{CONH}_{2}$ & MeS & $\mathrm{H}$ & $\mathrm{Me}$ & $\mathrm{H}$ & $\mathrm{Pd}-\mathrm{C} / \mathrm{MePh} / \Delta / 100 \mathrm{~h}$ & 48 & [31] \\
\hline $12 a$ & $\mathrm{E} / \mathrm{E}$ & $\mathrm{H}$ & MeS & $\mathrm{H}$ & $\mathrm{H}$ & $\mathrm{H}$ & $\mathrm{Pd}-\mathrm{C} / \mathrm{MePh} / \Delta / 100 \mathrm{~h}$ & 38 & [29] \\
\hline $12 b$ & $\mathrm{E} / \mathrm{E}$ & $\mathrm{H}$ & MeS & $\mathrm{H}$ & $\mathrm{H}$ & $\mathrm{Me}$ & $\mathrm{Pd}-\mathrm{C} / \mathrm{MePh} / \Delta / 100 \mathrm{~h}$ & 40 & [29] \\
\hline $12 \mathrm{c}$ & $\mathrm{E} / \mathrm{E}$ & $\mathrm{H}$ & $\mathrm{MeS}$ & $\mathrm{Me}$ & $\mathrm{H}$ & $\mathrm{Me}$ & $\mathrm{Pd}-\mathrm{C} / \mathrm{MePh} / \Delta / 100 \mathrm{~h}$ & 49 & [29] \\
\hline $13 a$ & $\mathrm{E} / \mathrm{E}$ & $\mathrm{H}$ & $\mathrm{H}$ & $\mathrm{H}$ & $\mathrm{NMe}_{2}$ & $\mathrm{H}$ & $\mathrm{Pd}-\mathrm{C} / \mathrm{MePh} / \Delta$ & 32 & [2] \\
\hline $13 b$ & $\mathrm{E} / \mathrm{E}$ & $\mathrm{H}$ & $\mathrm{MeS}$ & $\mathrm{H}$ & $\mathrm{NMe}_{2}$ & $\mathrm{H}$ & $\mathrm{Pd}-\mathrm{C} / \mathrm{MePh} / \Delta$ & 17 & [2] \\
\hline $14 a$ & $E^{\prime} / E^{\prime b}$ & $\mathrm{~F}$ & p-Brh & $\mathrm{H}$ & $\mathrm{H}$ & $\mathrm{H}$ & $\mathrm{Cu}(\mathrm{OAc})_{2} / \mathrm{PhMe} / \Delta / 5 \mathrm{~h}$ & 63 & [34] \\
\hline $14 b$ & $E^{\prime} / E^{\prime}$ & $\mathrm{F}$ & $\mathrm{p}-\mathrm{MeOPh}$ & $\mathrm{H}$ & $\mathrm{H}$ & $\mathrm{H}$ & $\mathrm{Cu}(\mathrm{OAc})_{2} / \mathrm{PhMe} / \Delta / 8 \mathrm{~h}$ & 70 & [34] \\
\hline 15 & $\mathrm{E} / \mathrm{H}$ & $\mathrm{E}$ & $\mathrm{Me}$ & $\mathrm{H}$ & $\mathrm{H}$ & $\mathrm{H}$ & $\mathrm{Pd}-\mathrm{C} / \mathrm{PhH} / \Delta / 24 \mathrm{~h}$ & $\mathrm{NG}^{\mathrm{c}}$ & [39] \\
\hline $16 a$ & $\mathrm{E} / \mathrm{H}$ & 2-Py & $\mathrm{Me}$ & $\mathrm{H}$ & $\mathrm{H}$ & $\mathrm{H}$ & $\mathrm{No} / \mathrm{NO}_{2} \mathrm{Ph} / \Delta / 20 \mathrm{~h}$ & 43 & [40] \\
\hline $16 b$ & $\mathrm{E} / \mathrm{E}$ & 2-Py & $\mathrm{Me}$ & $\mathrm{H}$ & $\mathrm{H}$ & $\mathrm{H}$ & $\mathrm{No} / \mathrm{NO}_{2} \mathrm{Ph} / \Delta / 20 \mathrm{~h}$ & 47 & [40] \\
\hline 17 & $\mathrm{E} / \mathrm{E}$ & $\mathrm{H}$ & $\mathrm{Me}$ & o-OHPhCO & $\mathrm{H}$ & $\mathrm{COMe}$ & $\mathrm{Pd}-\mathrm{C} / \mathrm{MePh} / \Delta / 16 \mathrm{~h}$ & 54 & [35] \\
\hline 18 & $\mathrm{E} / \mathrm{H}$ & $\mathrm{H}$ & $\mathrm{t}-\mathrm{Bu}$ & $\mathrm{H}$ & $\mathrm{H}$ & $\mathrm{H}$ & $\mathrm{O}_{2} / \mathrm{MePh} / \Delta / 4 \mathrm{~h}$ & 79 & [36] \\
\hline 19 & $\mathrm{Me}_{2} \mathrm{~B} / \mathrm{H}$ & $\mathrm{H}$ & $\mathrm{H}$ & $\mathrm{H}$ & $\mathrm{H}$ & $\mathrm{H}$ & (1) $\mathrm{MePh} / \Delta / 3 \mathrm{~d}$ (2) DDQ & 55 & [37] \\
\hline $20 a$ & $\mathrm{E} / \mathrm{E}$ & $\mathrm{H}$ & Styryl & $\mathrm{H}$ & $\mathrm{H}$ & $\mathrm{H}$ & $\mathrm{No} / \mathrm{PhMe} / 50^{\circ} / 31 \mathrm{~h}$ & 44 & [38] \\
\hline $20 b$ & $\mathrm{E} / \mathrm{H}$ & $\mathrm{H}$ & Styryl & $\mathrm{H}$ & $\mathrm{H}$ & $\mathrm{H}$ & $\mathrm{No} / \mathrm{PhMe} / 20^{\circ} / 120 \mathrm{~h}$ & 64 & [38] \\
\hline
\end{tabular}

1-Methoxycarbonyl indolizine 15 (Table 1) was converted to cyclazine in order to make cyclophane [39], but this methodology failed. Finally, cyclazines 21c,d were obtained from bis-indolizinylethanes 21a,b $(\mathrm{R}=\mathrm{Me}, \mathrm{t}-\mathrm{Bu})$ and further converted to cyclophanes [39], Scheme 5 .<smiles>[R]c1cn2ccccc2c1CCc1c([R])cn2ccccc12</smiles>

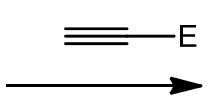<smiles>[R]c1c(CCc2c([R])c3cc(F)c4cccc2n43)c2cccc3c(F)cc1n32</smiles>

Scheme 5. Synthesis of bis-cyclazinylethanes from bis-indolizinylethanes.

1-(2-Pyridyl)indolizine in reaction with acetylenes $\mathbf{1 6} \mathbf{a}, \mathbf{b}$ formed cyclazine [40], Table 1. The product was converted to indolizino-cyclazine 16c, and DMAD was added for the second time giving bi-cyclazine $16 \mathbf{d}$ ( $20 \mathrm{~h}$ in boiling xylene without catalyst) with a yield of $30 \%$, Scheme 6.

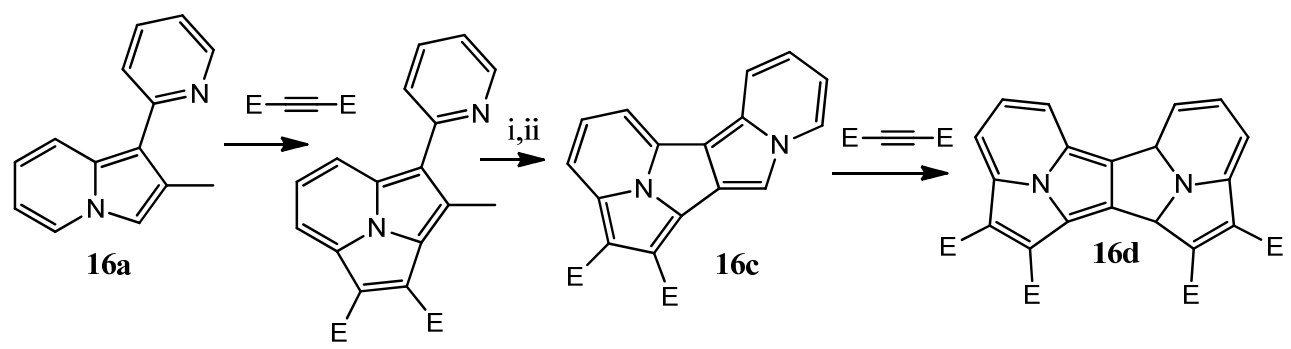

Scheme 6. i N-bromsuccinimide. $6 \mathrm{~h}$; ii $\mathrm{K}_{2} \mathrm{CO}_{3}$.

Analogous structure bearing 2- $\mathrm{CO}_{2}$ Et group 22a [41] was converted to condensed indolizino-cyclazinone structure $\mathbf{2 2 b}$. from which cyclazino-cyclazinone 22c was obtained with the yield $73 \%\left(\mathrm{Pd} / \mathrm{C}, \mathrm{NO}_{2} \mathrm{Ph}, 20 \mathrm{~h}\right)$, scheme 7. 


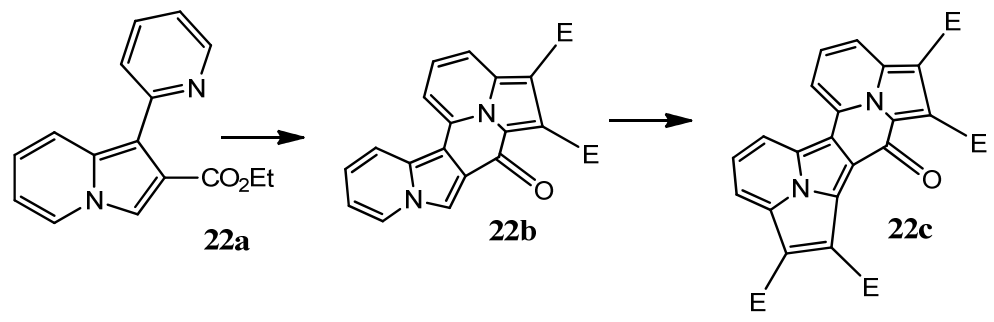

Scheme 7. Synthesis of cyclazino-cyclazinone.

A Japanese group made an effort to prepare cyclazines from 1,8-cycloannelated indolizines 23 [42-44] containing propylene and butylene bridges, Scheme 8 , Table 2 . The major finding was the use of DDQ in reaction with dibenzoyl acetylene (DBZA) under extremely mild conditions. Further studies on oxo-derivatives 24 [45] allowed to make cyclazine bearing (in 1 and 8 positions) oxo-propyl group.<smiles>[R]c1cn2cccc([R2])c2c1[R]</smiles>

23 - 25

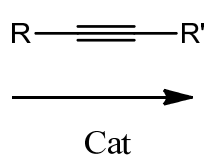

Cat<smiles>[R]c1c([R])c2c([R])c([R])c3c([R])ccc1n32</smiles>

Scheme 8. Synthesis of cyclazines from 1,8-cycloannelated indolizines (see Table 2).

Table 2. Substituents and yields of cycloaddition of acetylenes to indolizines 23-25 annelated by a ring across the positions $\mathrm{C} 1$ and C-8 (Scheme 8).

\begin{tabular}{|c|c|c|c|c|c|c|}
\hline $\mathbf{N}$ & $\mathbf{R} / \mathbf{R}^{\prime}$ & R8-R1 & R2 & Cat/Solv/T $T^{\circ} /$ Time & Yield \% & Ref. \\
\hline $23 a$ & $\mathrm{E} / \mathrm{H}$ & $\left(\mathrm{CH}_{2}\right)_{4}$ & $\mathrm{Me}$ & $\mathrm{Pd}-\mathrm{C} / \mathrm{PhMe} / \Delta / 50 \mathrm{~h}$ & 75 & [42] \\
\hline $23 b$ & $\mathrm{E} / \mathrm{H}$ & $\left(\mathrm{CH}_{2}\right)_{3}$ & $\mathrm{Me}$ & $\mathrm{Pd}-\mathrm{C} / \mathrm{PhMe} / \Delta / 50 \mathrm{~h}$ & 75 & [42] \\
\hline $23 c$ & $\mathrm{E} / \mathrm{E}$ & $\left(\mathrm{CH}_{2}\right)_{4}$ & $\mathrm{Me}$ & $\mathrm{Pd}-\mathrm{C} / \mathrm{PhMe} / \Delta / 50 \mathrm{~h}$ & 85 & [42] \\
\hline $23 d$ & $\mathrm{E} / \mathrm{E}$ & $\left(\mathrm{CH}_{2}\right)_{3}$ & $\mathrm{Ph}$ & $\mathrm{Pd}-\mathrm{C} / \mathrm{PhMe} / \Delta / 50 \mathrm{~h}$ & 58 & [42] \\
\hline $23 d$ & $\mathrm{E} / \mathrm{E}$ & $\left(\mathrm{CH}_{2}\right)_{4}$ & $\mathrm{Ph}$ & $\mathrm{Pd}-\mathrm{C} / \mathrm{PhMe} / \Delta / 50 \mathrm{~h}$ & 61 & [42] \\
\hline $23 e$ & $\mathrm{E} / \mathrm{E}$ & $\left(\mathrm{CH}_{2}\right)_{3}$ & $\mathrm{Me}$ & $\mathrm{Pd}-\mathrm{C} / \mathrm{PhMe} / \Delta / 50 \mathrm{~h}$ & 77 & [42] \\
\hline $24 f$ & $\mathrm{COPh} / \mathrm{COPh}$ & $\left(\mathrm{CH}_{2}\right)_{4}$ & $\mathrm{Me}$ & $\mathrm{DDQ} / \mathrm{THF} / 0^{\circ} / 1 \mathrm{~h}$ & 86 & [42] \\
\hline $23 g$ & $\mathrm{COPh} / \mathrm{COPh}$ & $\left(\mathrm{CH}_{2}\right)_{4}$ & $\mathrm{Ph}$ & $\mathrm{DDQ} / \mathrm{THF} / 0^{\circ} / 1 \mathrm{~h}$ & 92 & [42] \\
\hline $23 \mathrm{~h}$ & $\mathrm{COPh} / \mathrm{COPh}$ & $\left(\mathrm{CH}_{2}\right)_{3}$ & $\mathrm{Me}$ & $\mathrm{DDQ} / \mathrm{THF} / 0^{\circ} / 1 \mathrm{~h}$ & 68 & [42] \\
\hline $23 \mathbf{i}$ & $\mathrm{COPh} / \mathrm{COPh}$ & $\left(\mathrm{CH}_{2}\right)_{3}$ & $\mathrm{Ph}$ & $\mathrm{DDQ} / \mathrm{THF} / 0^{\circ} / 1 \mathrm{~h}$ & 52 & [42] \\
\hline $24 a$ & $\mathrm{E} / \mathrm{E}$ & $\mathrm{O}=\mathrm{C}\left(\mathrm{CH}_{2}\right)_{2}$ & $\mathrm{Ph}$ & $\mathrm{DDQ} / \mathrm{MePh} / \Delta / 2 \mathrm{~h}$ & 92 & [45] \\
\hline $24 b$ & $\mathrm{E} / \mathrm{E}$ & $\mathrm{O}=\mathrm{C}\left(\mathrm{CH}_{2}\right)_{2}$ & $E^{\prime}$ & $\mathrm{DDQ} / \mathrm{MePh} / \Delta / 2 \mathrm{~h}$ & 98 & [45] \\
\hline $25 a$ & $\mathrm{COPh} / \mathrm{COPh}$ & $\mathrm{CH}_{2}-\mathrm{NE}^{\prime}-\mathrm{CH}_{2}$ & $\mathrm{H}$ & $\mathrm{DDQ} / \mathrm{THF} / 0^{\circ} / 10 \mathrm{~m}$ & 66 & [46] \\
\hline $25 b$ & $\mathrm{COPh} / \mathrm{COPh}$ & $\mathrm{CH}_{2}-\mathrm{NE}^{\prime}-\left(\mathrm{CH}_{2}\right)_{2}$ & $\mathrm{H}$ & $\mathrm{DDQ} / \mathrm{THF} / 0^{\circ} / 10 \mathrm{~m}$ & 84 & [46] \\
\hline
\end{tabular}

Finally, fused indolizines $\mathbf{2 5}$ with saturated piperidyl or hexamethyleneimine bridges across 1,8-positions were [46] prepared and involved in cycloaddition with DBZA giving expected cyclazines, Scheme 9. Table 2. However, an attempt to perform similar reaction with DMAD caused cyclazine formation with unsaturated azepine ring. 


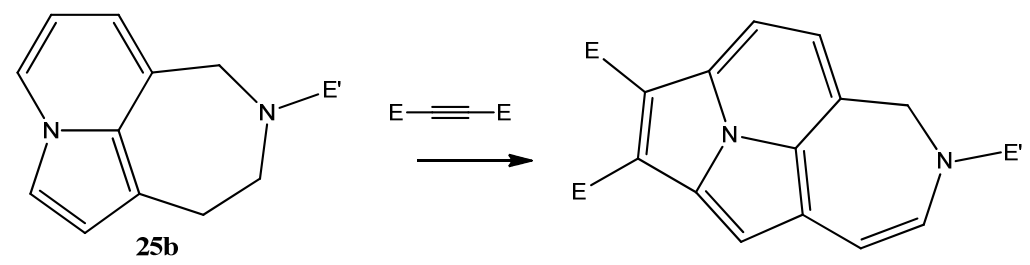

Scheme 9. Abnormal cycloaddition to 1,8-cycloannelated indolizine combined with dehydrogenation.

Novel reaction conditions were found for cycloaddition reaction, so that the role of oxidant was played by $\mathrm{O}_{2}$ in presence of $\mathrm{Pd}(\mathrm{OAc})_{2}$ [47], Scheme 10. Many 1-alkoxycarbonyl derivatives (26a-1) were involved in the reaction with acetylenes of the type $\mathrm{ArC} \equiv \mathrm{CAr}$, Table 3.<smiles>[R]c1cn2ccccc2c1[R]</smiles><smiles>[R]c1c([R])c2c([Al])c([Al])c3cccc1n32</smiles>

Scheme 10. $10 \mathrm{~mol} \% \mathrm{Pd}(\mathrm{OAc})_{2}, \mathrm{DMSO}, \mathrm{O}_{2}(1 \mathrm{~atm})$, no base.

Table 3. Substituents and yields of cycloaddition of diarylalkynes to indolizines (Scheme 10).

\begin{tabular}{|c|c|c|c|c|c|c|}
\hline $\mathbf{N}$ & $\mathbf{R}$ & $\mathbf{R}^{\prime}$ & R1 & $\mathbf{R} 2$ & Yield \% & Ref. \\
\hline $26 a$ & $\mathrm{Ph}$ & $\mathrm{Ph}$ & $E^{\prime}$ & $E^{\prime}$ & 92 & [47] \\
\hline $26 b$ & 4-MePh & 4-MePh & $\mathrm{E}$ & $\mathrm{H}$ & 88 & [47] \\
\hline $26 c$ & $\mathrm{Ph}$ & $\mathrm{Ph}$ & $\mathrm{E}$ & $\mathrm{E}$ & 98 & [47] \\
\hline $26 d$ & 4-FPh & 4-FPh & $\mathrm{E}$ & $\mathrm{E}$ & 85 & [47] \\
\hline $26 \mathrm{e}$ & 3-BrPh & 3-BrPh & $\mathrm{E}$ & $\mathrm{E}$ & 56 & [47] \\
\hline $26 f$ & $4-\mathrm{NO}_{2} \mathrm{Ph}$ & 4-MeOPh & E & $\mathrm{E}$ & $80(6: 1)$ & [47] \\
\hline $26 \mathrm{~g}$ & $4-\mathrm{NO}_{2} \mathrm{Ph}$ & $\mathrm{Ph}$ & E & $\mathrm{E}$ & $68(20: 1)$ & [47] \\
\hline $26 h$ & 4-FPh & 4-MeOPh & E & $\mathrm{E}$ & $91(2: 1)$ & [47] \\
\hline $26 \mathrm{i}$ & $\mathrm{C} \equiv \mathrm{CPh}$ & $\mathrm{Ph}$ & $\mathrm{E}$ & $\mathrm{E}$ & $41 ; 39$ & [47] \\
\hline $26 j$ & $\mathrm{Ph}$ & $\mathrm{Ph}$ & $\mathrm{CO}_{2} \mathrm{nBu}$ & $\mathrm{CO}_{2} \mathrm{nBu}$ & 79 & [47] \\
\hline $26 k$ & $\mathrm{Ph}$ & $\mathrm{Ph}$ & E & $\mathrm{Ph}$ & 20 * & [47] \\
\hline 261 & $\mathrm{Ph}$ & $\mathrm{Ph}$ & $\mathrm{CO}_{2} \mathrm{tBu}$ & $\mathrm{H}$ & 55 & [47] \\
\hline 261 & $\mathrm{Ph}$ & $\mathrm{Ph}$ & $\mathrm{H}$ & $\mathrm{E}$ & 70 & [47] \\
\hline $26 \mathrm{~m}$ & $\mathrm{Ph}$ & $\mathrm{Ph}$ & $\mathrm{CONMe}_{2}$ & $\mathrm{H}$ & 76 & [47] \\
\hline $26 n$ & $\mathrm{Ph}$ & $\mathrm{Ph}$ & $\mathrm{E}^{\prime}$ & $\mathrm{H}$ & 87 & [47] \\
\hline 260 & $\mathrm{Ph}$ & $\mathrm{Ph}$ & $\mathrm{E}$ & $\mathrm{H}$ & 90 & [47] \\
\hline $26 p$ & $\mathrm{Ph}$ & $\mathrm{Ph}$ & $\mathrm{CN}$ & $\mathrm{H}$ & 59 & [47] \\
\hline
\end{tabular}

A range of indolizine 27 smoothly underwent visible-light-induced intermolecular cyclization with internal alkynes with acceptor group to afford cyclazines in good to excellent yields with high regioselectivity [48], Scheme 11, Table 4.

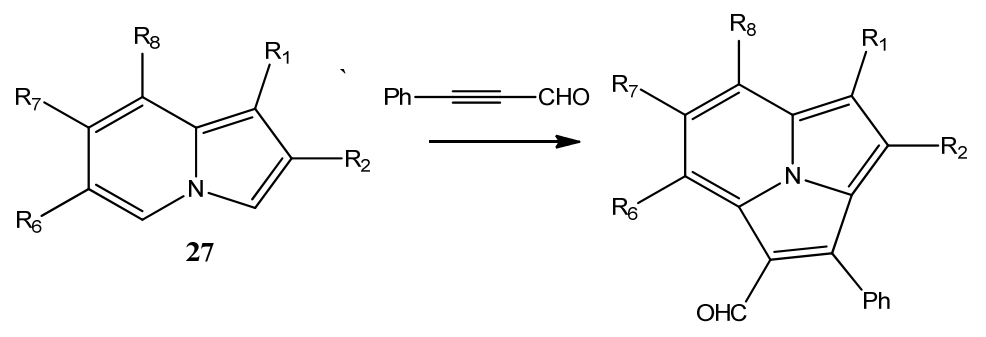

Scheme 11. Bengal rose KI, air, DMSO, 8 h, 20 W, blue LED. 
Table 4. Substituents and yields of photochemical cycloaddition to indolizines (Scheme 11).

\begin{tabular}{|c|c|c|c|c|c|c|c|c|c|}
\hline $\mathbf{N}$ & $\mathbf{R}^{\prime}$ & $\mathbf{R}^{\prime \prime}$ & R1 & R2 & R6 & R7 & R8 & Yield, \% & Ref. \\
\hline $27 a$ & $\mathrm{CHO}$ & $\mathrm{Ph}$ & $\mathrm{H}$ & $\mathrm{Ph}$ & $\mathrm{H}$ & $\mathrm{H}$ & $\mathrm{H}$ & 87 & [48] \\
\hline $27 b$ & $\mathrm{CHO}$ & $\mathrm{n}-\mathrm{C}_{5} \mathrm{H}_{11}$ & $\mathrm{H}$ & $\mathrm{Ph}$ & $\mathrm{H}$ & $\mathrm{H}$ & $\mathrm{H}$ & 61 & [48] \\
\hline $27 c$ & $\mathrm{CHO}$ & 2-Thienyl & $\mathrm{H}$ & $\mathrm{Ph}$ & $\mathrm{H}$ & $\mathrm{H}$ & $\mathrm{H}$ & 66 & [48] \\
\hline $27 d$ & $\mathrm{CHO}$ & 3-Cl-Ph & $\mathrm{H}$ & $\mathrm{Ph}$ & $\mathrm{H}$ & $\mathrm{H}$ & $\mathrm{H}$ & 79 & [48] \\
\hline $27 \mathrm{e}$ & $\mathrm{CHO}$ & 3-Ac-Ph & $\mathrm{H}$ & $\mathrm{Ph}$ & $\mathrm{H}$ & $\mathrm{H}$ & $\mathrm{H}$ & 58 & [48] \\
\hline $27 f$ & $\mathrm{CHO}$ & 4-Me-Ph & $\mathrm{H}$ & $\mathrm{Ph}$ & $\mathrm{H}$ & $\mathrm{H}$ & $\mathrm{H}$ & 77 & [48] \\
\hline $27 \mathrm{~g}$ & $\mathrm{CHO}$ & $3,4-\mathrm{Me}_{2} \mathrm{Ph}$ & $\mathrm{H}$ & $\mathrm{Ph}$ & $\mathrm{H}$ & $\mathrm{H}$ & $\mathrm{H}$ & 57 & [48] \\
\hline $27 \mathrm{~h}$ & $\mathrm{CHO}$ & 2-Naphtyl & $\mathrm{H}$ & $\mathrm{Ph}$ & $\mathrm{H}$ & $\mathrm{H}$ & $\mathrm{H}$ & 59 & [48] \\
\hline $27 \mathbf{i}$ & $\mathrm{COPh}$ & $\mathrm{Ph}$ & $\mathrm{H}$ & $\mathrm{Ph}$ & $\mathrm{H}$ & $\mathrm{H}$ & $\mathrm{H}$ & 58 & [48] \\
\hline $27 j$ & $\mathrm{Ph}$ & $\mathrm{Ph}$ & $\mathrm{H}$ & $\mathrm{Ph}$ & $\mathrm{H}$ & $\mathrm{H}$ & $\mathrm{H}$ & 0 & [48] \\
\hline $27 k$ & $\mathrm{CHO}$ & $\mathrm{Ph}$ & $\mathrm{H}$ & 4-OMe-Ph & $\mathrm{H}$ & $\mathrm{H}$ & $\mathrm{H}$ & 70 & [48] \\
\hline 271 & $\mathrm{CHO}$ & $\mathrm{Ph}$ & $\mathrm{H}$ & 4-F-Ph & $\mathrm{H}$ & $\mathrm{H}$ & $\mathrm{H}$ & 81 & [48] \\
\hline $27 \mathrm{~m}$ & $\mathrm{CHO}$ & $\mathrm{Ph}$ & $\mathrm{H}$ & $4-\mathrm{NO}_{2}-\mathrm{Ph}$ & $\mathrm{H}$ & $\mathrm{H}$ & $\mathrm{H}$ & 58 & [48] \\
\hline $27 n$ & $\mathrm{CHO}$ & $\mathrm{Ph}$ & $\mathrm{H}$ & 4-Br-Ph & $\mathrm{H}$ & $\mathrm{H}$ & $\mathrm{H}$ & 64 & [48] \\
\hline 270 & $\mathrm{CHO}$ & $\mathrm{Ph}$ & $\mathrm{H}$ & $4-\mathrm{CF}_{3}-\mathrm{Ph}$ & $\mathrm{H}$ & $\mathrm{H}$ & $\mathrm{H}$ & 71 & [48] \\
\hline 27 & $\mathrm{CHO}$ & $\mathrm{Ph}$ & $\mathrm{H}$ & 2-F-Ph & $\mathrm{H}$ & $\mathrm{H}$ & $\mathrm{H}$ & 73 & [48] \\
\hline $27 p$ & $\mathrm{CHO}$ & $\mathrm{Ph}$ & $\mathrm{H}$ & 3-Me-Ph & $\mathrm{H}$ & $\mathrm{H}$ & $\mathrm{H}$ & 63 & [48] \\
\hline $27 q$ & $\mathrm{CHO}$ & $\mathrm{Ph}$ & $\mathrm{H}$ & $3,4-\mathrm{Cl}_{2}-\mathrm{Ph}$ & $\mathrm{H}$ & $\mathrm{H}$ & $\mathrm{H}$ & 58 & [48] \\
\hline $27 \mathrm{r}$ & $\mathrm{CHO}$ & $\mathrm{Ph}$ & $\mathrm{H}$ & $2,4-\mathrm{Cl}_{2}-\mathrm{Ph}$ & $\mathrm{H}$ & $\mathrm{H}$ & $\mathrm{H}$ & 78 & [48] \\
\hline $27 s$ & $\mathrm{CHO}$ & $\mathrm{Ph}$ & $\mathrm{H}$ & $3,4-(\mathrm{OMe})_{2}-\mathrm{Ph}$ & $\mathrm{H}$ & $\mathrm{H}$ & $\mathrm{H}$ & 84 & [48] \\
\hline $27 t$ & $\mathrm{CHO}$ & $\mathrm{Ph}$ & $\mathrm{H}$ & 1,3-Benzo-dioxolyl-5 & $\mathrm{H}$ & $\mathrm{H}$ & $\mathrm{H}$ & 67 & [48] \\
\hline $27 \mathbf{u}$ & $\mathrm{CHO}$ & $\mathrm{Ph}$ & $\mathrm{H}$ & Furyl & $\mathrm{H}$ & $\mathrm{H}$ & $\mathrm{H}$ & 62 & [48] \\
\hline $27 \mathbf{v}$ & $\mathrm{CHO}$ & $\mathrm{Ph}$ & $\mathrm{H}$ & 2-Naphtyl & $\mathrm{H}$ & $\mathrm{H}$ & $\mathrm{H}$ & 60 & [48] \\
\hline $27 w$ & $\mathrm{CHO}$ & $\mathrm{Ph}$ & $E^{\prime}$ & $\mathrm{H}$ & $\mathrm{H}$ & $\mathrm{H}$ & $\mathrm{H}$ & 59 & [48] \\
\hline $27 x$ & $\mathrm{CHO}$ & $\mathrm{Ph}$ & $\mathrm{H}$ & $\mathrm{Ph}$ & Et & $\mathrm{H}$ & $\mathrm{H}$ & 61 & [48] \\
\hline $27 y$ & $\mathrm{CHO}$ & $\mathrm{Ph}$ & $\mathrm{H}$ & $\mathrm{Ph}$ & $\mathrm{H}$ & $\mathrm{Me}$ & $\mathrm{H}$ & 73 & [48] \\
\hline $27 z$ & $\mathrm{CHO}$ & $\mathrm{Ph}$ & $\mathrm{H}$ & $\mathrm{Ph}$ & $\mathrm{H}$ & OMe & $\mathrm{H}$ & 70 & [48] \\
\hline $27 a 1$ & $\mathrm{CHO}$ & $\mathrm{Ph}$ & $\mathrm{H}$ & $\mathrm{Ph}$ & $\mathrm{Me}$ & $\mathrm{H}$ & $\mathrm{Me}$ & 61 & [48] \\
\hline $27 \mathrm{~b} 1$ & $E^{\prime}$ & $\mathrm{H}$ & $\mathrm{H}$ & $\mathrm{Ph}$ & $\mathrm{H}$ & $\mathrm{H}$ & $\mathrm{H}$ & 88 & [48] \\
\hline $27 \mathrm{c} 1$ & $\mathrm{E}^{\prime}$ & $\mathrm{H}$ & $\mathrm{H}$ & $\mathrm{Ph}$ & $\mathrm{H}$ & $\mathrm{H}$ & $\mathrm{H}$ & 70 & [48] \\
\hline $27 d 1$ & $E^{\prime}$ & $E^{\prime}$ & $\mathrm{H}$ & $\mathrm{Ph}$ & $\mathrm{H}$ & $\mathrm{H}$ & $\mathrm{H}$ & 77 & [48] \\
\hline $27 \mathrm{e} 1$ & $E^{\prime}$ & $E^{\prime}$ & $\mathrm{H}$ & $\mathrm{Ph}$ & $\mathrm{H}$ & $\mathrm{H}$ & $\mathrm{H}$ & 70 & [48] \\
\hline
\end{tabular}

An efficient visible-light-induced intermolecular [8+2] alkenylation-cyclization process was developed for indolizines 28 [49], Scheme 12, Table 5. In this reaction alkene (not alkyne) formed cyclazine derivatives with oxygen as an oxidant via cascade reaction.<smiles>[R]C=CC(C)C</smiles>

Scheme 12. Bengal Rose, TFA, $\mathrm{CH}_{2} \mathrm{Cl}_{2}, \mathrm{O}_{2}, 10 \mathrm{~h}, 20 \mathrm{~W}$ blue LED.

Table 5. Substituents and yields of oxidative cycloaddition of alkenes to indolizines (Scheme 12).

\begin{tabular}{ccccccccc}
\hline $\mathbf{N}$ & $\mathbf{R}$ & $\mathbf{R}$ & $\mathbf{R 5}$ & $\mathbf{R 6}$ & $\mathbf{R} 7$ & $\mathbf{R} 8$ & Yield, \% & Ref. \\
\hline $\mathbf{2 8 a}$ & $\mathrm{E}$ & $\mathrm{Ph}$ & $\mathrm{H}$ & $\mathrm{H}$ & $\mathrm{H}$ & $\mathrm{H}$ & 80 & {$[49]$} \\
$\mathbf{2 8 b}$ & $\mathrm{CO}_{2} \mathrm{nBu}$ & $\mathrm{Ph}$ & $\mathrm{H}$ & $\mathrm{H}$ & $\mathrm{H}$ & $\mathrm{H}$ & 69 & {$[49]$} \\
$\mathbf{2 8 c}$ & $\mathrm{CO}_{2} \mathrm{CH}_{2} \mathrm{CHOH}$ & $\mathrm{Ph}$ & $\mathrm{H}$ & $\mathrm{H}$ & $\mathrm{H}$ & $\mathrm{H}$ & 68 & {$[49]$} \\
$\mathbf{2 8 d}$ & $\mathrm{CONH}-\mathrm{Bu}$ & $\mathrm{Ph}$ & $\mathrm{H}$ & $\mathrm{H}$ & $\mathrm{H}$ & $\mathrm{H}$ & 67 & {$[49]$} \\
$\mathbf{2 8}$ & $2-\mathrm{Py}$ & $\mathrm{Ph}$ & $\mathrm{H}$ & $\mathrm{H}$ & $\mathrm{H}$ & $\mathrm{H}$ & 65 & {$[49]$} \\
$\mathbf{2 8 f}$ & $\mathrm{E}$ & $\mathrm{Ph}$ & $\mathrm{Me}$ & $\mathrm{H}$ & $\mathrm{H}$ & $\mathrm{H}$ & 78 & {$[49]$} \\
$\mathbf{2 8 g}$ & $2-\mathrm{Py}$ & $\mathrm{Ph}$ & $\mathrm{H}$ & $\mathrm{H}$ & $\mathrm{H}$ & $\mathrm{Me}$ & 69 & {$[49]$} \\
$\mathbf{2 8 h}$ & $\mathrm{E}$ & $\mathrm{Ph}$ & $\mathrm{H}$ & $\mathrm{H}$ & $\mathrm{Me}$ & $\mathrm{H}$ & 77 & {$[49]$} \\
$\mathbf{2 8 i}$ & $\mathrm{E}$ & $\mathrm{Ph}$ & $\mathrm{H}$ & $\mathrm{Et}$ & $\mathrm{H}$ & $\mathrm{H}$ & 70 & {$[49]$} \\
$\mathbf{2 8 j}$ & $\mathrm{E}$ & $4-\mathrm{FPh}$ & $\mathrm{H}$ & $\mathrm{H}$ & $\mathrm{H}$ & $\mathrm{H}$ & 75 & {$[49]$} \\
$\mathbf{2 8 k}$ & $\mathrm{E}$ & $4-\mathrm{BrPh}$ & $\mathrm{H}$ & $\mathrm{H}$ & $\mathrm{H}$ & $\mathrm{H}$ & 78 & {$[49]$} \\
$\mathbf{2 8 1}$ & $\mathrm{E}$ & $4-\mathrm{MePh}$ & $\mathrm{H}$ & $\mathrm{H}$ & $\mathrm{H}$ & $\mathrm{H}$ & 74 & {$[49]$} \\
$\mathbf{2 8 m}$ & $\mathrm{E}$ & $4-\mathrm{MeOPh}$ & $\mathrm{H}$ & $\mathrm{H}$ & $\mathrm{H}$ & $\mathrm{H}$ & 71 & {$[49]$} \\
\hline
\end{tabular}


Table 5. Cont.

\begin{tabular}{|c|c|c|c|c|c|c|c|c|}
\hline $\mathbf{N}$ & $\mathbf{R}$ & R2 & R5 & R6 & R7 & R8 & Yield, \% & Ref \\
\hline $28 n$ & E & 3-FPh & $\mathrm{H}$ & $\mathrm{H}$ & $\mathrm{H}$ & $\mathrm{H}$ & 75 & [49] \\
\hline 280 & E & 3-ClPh & $\mathrm{H}$ & $\mathrm{H}$ & $\mathrm{H}$ & $\mathrm{H}$ & 66 & [49] \\
\hline $28 p$ & E & 3-BrPh & $\mathrm{H}$ & $\mathrm{H}$ & $\mathrm{H}$ & $\mathrm{H}$ & 70 & [49] \\
\hline $28 q$ & $\mathrm{E}$ & 3-MePh & $\mathrm{H}$ & $\mathrm{H}$ & $\mathrm{H}$ & $\mathrm{H}$ & 71 & [49] \\
\hline $28 \mathrm{r}$ & 2-Py & 3-MePh & $\mathrm{H}$ & $\mathrm{H}$ & $\mathrm{H}$ & $\mathrm{H}$ & 66 & [49] \\
\hline $28 \mathrm{~s}$ & E & 3-MeOPh & $\mathrm{H}$ & $\mathrm{H}$ & $\mathrm{H}$ & $\mathrm{H}$ & 68 & [49] \\
\hline $28 \mathrm{t}$ & E & 3-FPh & $\mathrm{H}$ & $\mathrm{H}$ & $\mathrm{H}$ & $\mathrm{H}$ & 65 & [49] \\
\hline $28 u$ & E & $3,4-\mathrm{Cl}_{2} \mathrm{Ph}$ & $\mathrm{H}$ & $\mathrm{H}$ & $\mathrm{H}$ & $\mathrm{H}$ & 70 & [49] \\
\hline $28 v$ & E & $3,4-(\mathrm{MeO})_{2} \mathrm{Ph}$ & $\mathrm{H}$ & $\mathrm{H}$ & $\mathrm{H}$ & $\mathrm{H}$ & 66 & [49] \\
\hline $28 x$ & E & 2-Thienyl & $\mathrm{H}$ & $\mathrm{H}$ & $\mathrm{H}$ & $\mathrm{H}$ & 69 & [49] \\
\hline
\end{tabular}

Annulations of 1-cyanoIndolizine with unsaturated carboxylic acids 29a-f was observed during the catalysis with $\mathrm{Pd}(\mathrm{OAc})_{2}$ [50], via similar cascade reaction Scheme 13, Table 6.<smiles>N#Cc1ccn2ccccc12</smiles><smiles>CC=CC(=O)O</smiles><smiles>[R]c1c(C(=O)O)c2cccc3c(C#N)cc1n32</smiles>

Scheme 13. $10 \mathrm{~mol} \% \mathrm{Pd}(\mathrm{OAc})_{2}, 1$ eq benzoquinone, $\mathrm{O}_{2}, 2$ eq KOAc, DMF. $120^{\circ}, 12 \mathrm{~h}$.

Table 6. Yields of catalytic cycloaddition of acrylates (Scheme 13) to 1-cyanoindolizine.

\begin{tabular}{cccc}
\hline $\mathbf{N}$ & $\mathbf{R}$ & Yield, $\%$ & Ref. \\
\hline 29a & $\mathrm{Me}$ & 39 & {$[50]$} \\
$\mathbf{2 9 b}$ & $\mathrm{Ph}$ & 52 & {$[50]$} \\
29c & 4-Cl-Ph & 46 & {$[50]$} \\
29e & 4-OMePh & 59 & {$[50]$} \\
29f & 1-Naphtyl & 45 & {$[50]$} \\
\hline
\end{tabular}

\section{Non-Catalytic Cycloaddition to 3- or 5-Substituted Indolizines}

If a leaving group $\mathrm{X}$ is located at position 3 or 5 of indolizine ring, cycloaddition reaction does not require a catalyst/oxidant for dehydrogenation, because the dihydrocyclazine intermediate can lose HX, Scheme 14.

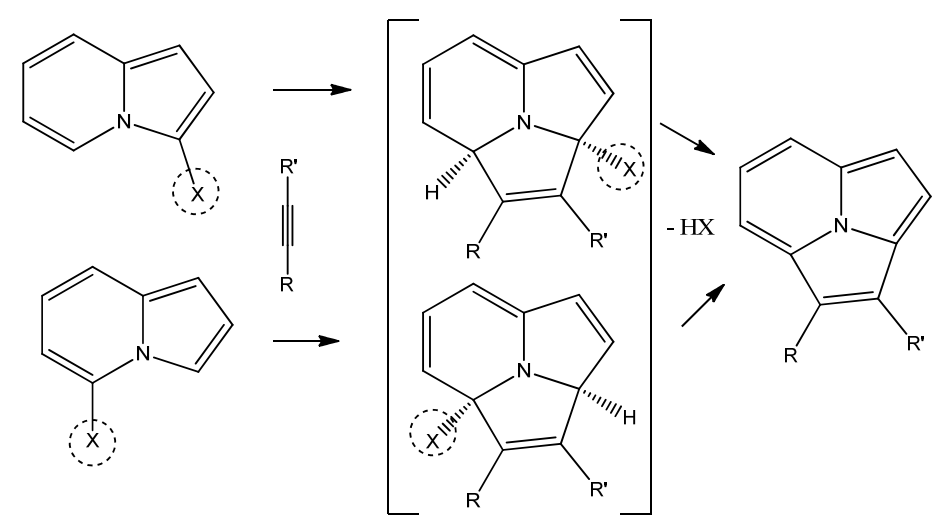

Scheme 14. Cycloaddition of indolizines with 3(5)-leaving groups.

Such groups $\mathrm{X}$ can be -OR or -OCOR. -SR, - $\mathrm{NR}_{2}$ or -NR-NR 2 , halogen and some others, Scheme 15. 
Table 7. Substituents, conditions and yields in cycloaddition of acetylenes to 3(5)-substituted indolizines giving cyclazines (Scheme 15).

\begin{tabular}{|c|c|c|c|c|c|c|c|c|c|c|}
\hline $\mathbf{N}$ & $\mathbf{R} / \mathbf{R}^{\prime}$ & R3/R5 & $\mathbf{R} 1$ & $\mathbf{R} 2$ & R6 & R7 & R8 & Cat/Solv/ $\mathrm{T}^{\circ} /$ Time & Yield \% & Ref. \\
\hline $30 a$ & $\mathrm{E} / \mathrm{H}$ & $\mathrm{OCOMe} / \mathrm{H}$ & $\mathrm{H}$ & $\mathrm{H}$ & $\mathrm{H}$ & $\mathrm{H}$ & $\mathrm{H}$ & $\mathrm{No} / \mathrm{Ac}_{2} \mathrm{O} / \Delta / 0.5 \mathrm{~h}$ & 86 & [51] \\
\hline $30 \mathrm{~b}$ & $\mathrm{E} / \mathrm{H}$ & $\mathrm{OCOEt} / \mathrm{H}$ & $\mathrm{H}$ & $\mathrm{H}$ & $\mathrm{H}$ & $\mathrm{H}$ & $\mathrm{H}$ & $\mathrm{No} / \mathrm{Ac}_{2} \mathrm{O} / \Delta / 0.5 \mathrm{~h}$ & 100 & [51] \\
\hline $30 c$ & $\mathrm{E} / \mathrm{H}$ & $\mathrm{OCOMe} / \mathrm{H}$ & $\mathrm{Ph}$ & $\mathrm{H}$ & $\mathrm{H}$ & $\mathrm{H}$ & $\mathrm{H}$ & $\mathrm{No} / \mathrm{Ac}_{2} \mathrm{O} / \Delta / 0.5 \mathrm{~h}$ & 90 & [51] \\
\hline $30 \mathrm{~d}$ & $\mathrm{E} / \mathrm{H}$ & $\mathrm{OCOMe} / \mathrm{H}$ & $\mathrm{CH}_{2} \mathrm{E}^{\prime}$ & $E^{\prime}$ & $\mathrm{H}$ & $\mathrm{H}$ & $\mathrm{H}$ & $\mathrm{No} / \mathrm{Ac}_{2} \mathrm{O} / \Delta / 0.5 \mathrm{~h}$ & 100 & [51] \\
\hline 31 & $\mathrm{E} / \mathrm{E}$ & i-PrS/H & i-PrS & i-PrS & $\mathrm{H}$ & $\mathrm{H}$ & $\mathrm{H}$ & $\mathrm{No} / \mathrm{PhMe} / \Delta / 40 \mathrm{~h}$ & 96 & [52] \\
\hline $32 a$ & $\mathrm{E} / \mathrm{E}$ & $\mathrm{NE}^{\prime} \mathrm{NHE}^{\prime} / \mathrm{H}$ & $\mathrm{Ph}$ & $\mathrm{H}$ & $\mathrm{H}$ & $\mathrm{H}$ & $\mathrm{H}$ & $\mathrm{No} / \mathrm{PhH} / \Delta / 5 \mathrm{~h}$ & 5.6 & [53] \\
\hline $32 b$ & $\mathrm{E} / \mathrm{E}$ & $\mathrm{NE}^{\prime} \mathrm{NHE}^{\prime} / \mathrm{H}$ & $\mathrm{H}$ & $\mathrm{H}$ & $\mathrm{H}$ & $\mathrm{H}$ & $\mathrm{H}$ & $\mathrm{No} / \mathrm{PhH} / \Delta / 5 \mathrm{~h}$ & 32 & [53] \\
\hline $32 c$ & $\mathrm{E} / \mathrm{E}$ & $\mathrm{NE}^{\prime} \mathrm{NHE}^{\prime} / \mathrm{H}$ & $\mathrm{NE}^{\prime} \mathrm{NHE}^{\prime}$ & $\mathrm{H}$ & $\mathrm{H}$ & $\mathrm{H}$ & $\mathrm{H}$ & $\mathrm{No} / \mathrm{PhH} / \Delta / 5 \mathrm{~h}$ & 66 & [53] \\
\hline $33 a$ & $\mathrm{E} / \mathrm{E}$ & H/OTms & $\mathrm{H}$ & $\mathrm{H}$ & $\mathrm{OMe}$ & $\mathrm{OMe}$ & $\mathrm{OH}$ & $\mathrm{No} / \mathrm{PhMe} / \Delta / 23 \mathrm{~h}$ & 56 & [54] \\
\hline $33 b$ & $\mathrm{E} / \mathrm{E}$ & $\mathrm{H} / \mathrm{OTms}$ & $\mathrm{H}$ & $\mathrm{H}$ & $\mathrm{OMe}$ & $\mathrm{Ph}$ & $\mathrm{OH}$ & $\mathrm{No} / \mathrm{PhMe} / \Delta / 23 \mathrm{~h}$ & 79 & [54] \\
\hline $33 c$ & $\mathrm{E} / \mathrm{E}$ & $\mathrm{H} / \mathrm{OTms}$ & $\mathrm{H}$ & $\mathrm{H}$ & $\mathrm{OMe}$ & $\mathrm{n}-\mathrm{Bu}$ & $\mathrm{OH}$ & $\mathrm{No} / \mathrm{PhMe} / \Delta / 23 \mathrm{~h}$ & 52 & [54] \\
\hline $33 d$ & $\mathrm{E} / \mathrm{E}$ & H/OTms & $\mathrm{H}$ & $\mathrm{H}$ & $\mathrm{OMe}$ & $\mathrm{n}-\mathrm{BuC} \equiv \mathrm{C}$ & $\mathrm{OH}$ & $\mathrm{No} / \mathrm{PhMe} / \Delta / 23 \mathrm{~h}$ & 67 & [54] \\
\hline $33 e$ & $\mathrm{E} / \mathrm{E}$ & $\mathrm{H} / \mathrm{OTms}$ & $\mathrm{H}$ & $\mathrm{H}$ & $\mathrm{OMe}$ & $\mathrm{PhC} \equiv \mathrm{C}$ & $\mathrm{OH}$ & $\mathrm{No} / \mathrm{PhMe} / \Delta / 23 \mathrm{~h}$ & 66 & [54] \\
\hline $33 f$ & $\mathrm{E} / \mathrm{E}$ & H/OTms & $\mathrm{H}$ & $\mathrm{H}$ & $\mathrm{n}-\mathrm{Bu}$ & $\mathrm{n}-\mathrm{Bu}$ & $\mathrm{OH}$ & $\mathrm{No} / \mathrm{PhMe} / \Delta / 23 \mathrm{~h}$ & 53 & [54] \\
\hline 34 & $\mathrm{E} / \mathrm{E}$ & H/Morph & $\mathrm{H}$ & $\mathrm{p}-\mathrm{NO}_{2} \mathrm{Ph}$ & $\mathrm{H}$ & $\mathrm{H}$ & $\mathrm{H}$ & $\mathrm{No} / \mathrm{PhH} / \Delta / 16 \mathrm{~h}$ & 82 & [55] \\
\hline 35 & $\mathrm{E} / \mathrm{E}$ & $\mathrm{H} / \mathrm{Br}$ & $\mathrm{Me}$ & $\mathrm{t}-\mathrm{Bu}$ & $\mathrm{H}$ & $\mathrm{H}$ & $\mathrm{H}$ & $\mathrm{No} / \mathrm{PhMe} / 80^{\circ} / 2 \mathrm{~h}$ & 53 & [56] \\
\hline
\end{tabular}<smiles>[R]c1c([R])c2ccccn2c1[N+]#C</smiles><smiles>[R]c1c([N+]2([O-])CCCCC2)c2ccccn2c1[R]</smiles><smiles>[R]c1c([N+]#[W])c2ccccn2c1[N+]#[C-]</smiles><smiles>[R]c1c(O)c(C#C)n2cccc2c1O</smiles><smiles></smiles><smiles>[R]c1cc2n(c1)C1(Br)CCCCC1=CC=C2</smiles>

Scheme 15. Examples of 3(5)-substituted indolizines involved in cycloaddition (see Table 7).

Thus, 3-acyloxy indolizines $\mathbf{3 0 a - d}$ were converted to cyclazines with excellent yield [51]. Tris-1,2,3-(iso-propylthio)indolizine 31 also underwent such cycloaddition [52]. 3-Hydrazinesubstituted derivatives 32a-c lost the attaching group forming cyclazines [53]. Quite similarly behaved 5-substituted indolizines. After refluxing in aromatic solvents, 5-OTms indolizines 33a-f [54], 5-morpholyl 34 [55] and 5-bromo derivatives 35 [56] smoothly formed the expected cyclazine structures in the absence of catalyst.

\section{Features of Cycloaddition of 3-Cyano Indolizines and Their Benzo Derivatives}

3-CN-Indolizines are the structures that looked capable to react with acetylenes without catalyst due to probable loss of HCN from intermediate. In 1980 the Matsumoto group (together with L. Paquet) reported the first reaction of 3-CN-inolizines with DMAD [57], [58]. 3-Cyanindolizine 36a and its 6,8-dimethyl analog 36b with DMAD in refluxing toluene gave expected cyclazines, though in presence of Pd-C (Scheme 16, Table 8). The later group of Tominaga converted 2-MeS-derivatives of 3-CN-indolizines-37a,b to MeS-cyclazines (again in the presence of the same catalyst) [59] (Scheme 16, Table 8). 


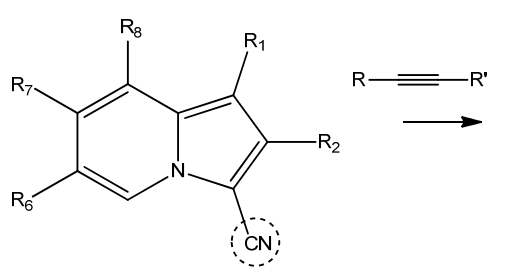<smiles>[R]c1c([R])c2c([R])c([R])c3c([R])c([R])c(c1[R])n23</smiles>

Scheme 16. Cycloaddition to 3-CN-substituted indolizines (see Table 8).

Table 8. Substituents, conditions and yields in cycloaddition of acetylene acetylenes to 3-CNsubstituted indolizines giving cyclazines (Scheme 16).

\begin{tabular}{|c|c|c|c|c|c|c|c|c|c|}
\hline $\mathbf{N}$ & $\mathbf{R} / \mathbf{R}^{\prime}$ & R1 & R2 & R6 & R7 & R8 & Cat/Solv/T ${ }^{\circ} /$ Time & Yield \% & Ref. \\
\hline $36 a$ & $\mathrm{E} / \mathrm{E}$ & $\mathrm{H}$ & $\mathrm{H}$ & $\mathrm{H}$ & $\mathrm{H}$ & $\mathrm{H}$ & $\mathrm{Pd}-\mathrm{C} / \mathrm{PhH} / \Delta / 24 \mathrm{~h}$ & 40 & [58] \\
\hline $36 b$ & $\mathrm{E} / \mathrm{E}$ & $\mathrm{H}$ & $\mathrm{H}$ & $\mathrm{Me}$ & $\mathrm{H}$ & $\mathrm{Me}$ & $\mathrm{Pd}-\mathrm{C} / \mathrm{PhH} / \Delta / 24 \mathrm{~h}$ & 25 & [58] \\
\hline $37 \mathbf{a}$ & $\mathrm{E} / \mathrm{E}$ & $\mathrm{H}$ & MeS & $\mathrm{H}$ & $\mathrm{H}$ & $\mathrm{H}$ & $\mathrm{Pd}-\mathrm{C} / \mathrm{PhH} / \Delta / 30 \mathrm{~h}$ & 22 & [59] \\
\hline $37 \mathrm{~b}$ & $\mathrm{E} / \mathrm{E}$ & $\mathrm{H}$ & $\mathrm{MeS}$ & $\mathrm{Me}$ & $\mathrm{H}$ & $\mathrm{Me}$ & $\mathrm{Pd}-\mathrm{C} / \mathrm{PhH} / \Delta / 30 \mathrm{~h}$ & 12 & [59] \\
\hline $38 a$ & $\mathrm{E} / \mathrm{E}$ & $\mathrm{H}$ & $\mathrm{H}$ & $\mathrm{H}$ & $\mathrm{H}$ & $\mathrm{H}$ & $\mathrm{Pd}^{\prime} \mathrm{C} / \mathrm{MePh} / \Delta / 24 \mathrm{~h}$ & 40 & [60] \\
\hline $38 b$ & $\mathrm{E} / \mathrm{E}$ & $\mathrm{H}$ & $\mathrm{H}$ & $\mathrm{H}$ & $\mathrm{Me}$ & $\mathrm{H}$ & $\mathrm{Pd} \mathrm{d}^{\prime} \mathrm{C} / \mathrm{MePh} / \Delta / 25-74 \mathrm{~h}$ & $10-7$ & {$[60]$} \\
\hline $38 \mathrm{c}$ & $\mathrm{E} / \mathrm{E}$ & $\mathrm{H}$ & $\mathrm{H}$ & $\mathrm{H}$ & $\mathrm{PhCH}_{2}$ & $\mathrm{H}$ & $\mathrm{Pd} \mathrm{d}^{\prime} \mathrm{C} / \mathrm{MePh} / \Delta / 30 \mathrm{~h}$ & 5 & [60] \\
\hline $38 d$ & $\mathrm{E} / \mathrm{E}$ & $\mathrm{H}$ & $\mathrm{H}$ & $\mathrm{H}$ & $\mathrm{Ph}$ & $\mathrm{H}$ & $\mathrm{Pd}{ }^{\prime} \mathrm{C} / \mathrm{MePh} / \Delta / 215 \mathrm{~h}$ & 13 & {$[60]$} \\
\hline $38 \mathrm{e}$ & $\mathrm{E} / \mathrm{E}$ & $\mathrm{H}$ & $\mathrm{H}$ & $\mathrm{Me}$ & $\mathrm{H}$ & $\mathrm{Me}$ & $\mathrm{Pd}^{\prime} \mathrm{C} / \mathrm{MePh} / \Delta / 77 \mathrm{~h}$ & 25 & {$[60]$} \\
\hline $38 \mathrm{f}$ & $\mathrm{E} / \mathrm{E}$ & $\mathrm{H}$ & $\mathrm{H}$ & $\mathrm{H}$ & $\mathrm{CN}$ & $\mathrm{H}$ & $\mathrm{Pd} \mathrm{d}^{\prime} \mathrm{C} / \mathrm{MePh} / \Delta / 260 \mathrm{~h}$ & 7 & [60] \\
\hline $38 \mathrm{~g}$ & $\mathrm{E} / \mathrm{E}$ & $\mathrm{H}$ & $\mathrm{H}$ & $\mathrm{H}$ & $\mathrm{E}$ & $\mathrm{H}$ & $\mathrm{Pd} \mathrm{d}^{\prime} \mathrm{C} / \mathrm{MePh} / \Delta / 336 \mathrm{~h}$ & 2 & [60] \\
\hline
\end{tabular}

The most dramatic story happened to another adduct of $\mathrm{CN}$-indolizines and DMAD. In 1980 the Matsumoto group found that 7-methyl- and 7-benzyl derivatives gave 1:2 adduct with proposed structure 39a [58], Scheme 17. Later the same group tested the reaction of 3-CN indolizines 38a-g in the presence and absence of a catalyst $[60,61]$, Table 8 . Finally, the structure of the 1:2 adduct formed without the catalyst was proved by X-ray, and it was unexpectedly styryl pyrrole 39b [60,61], Scheme 17 . Different mechanisms of benzene ring formation and E-group migration have been proposed.

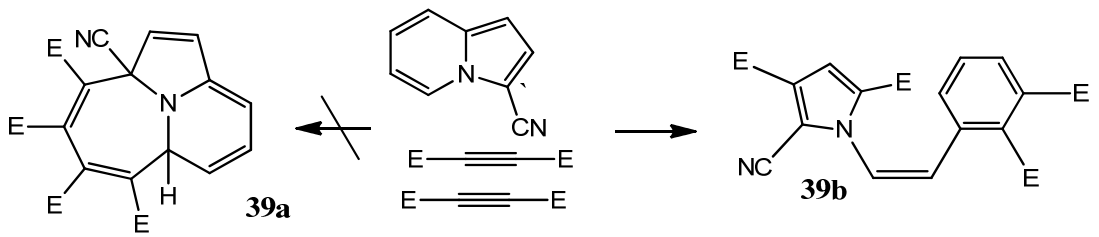

Scheme 17. Structure of 1:2 adduct of 3-cyanoindolizine and DMAD.

Cyano-derivative of benzo[a]indolizine is easily available from pyridinium-dicyanme thylide and dehydrobenzene. Matsumoto first published the results of cycloaddition of dibenzoylacetylene to the structures 40a-d (Scheme 18, Table 9) [62,63]. Again, the reaction required a catalyst. Tominaga group made this cycloaddition 41 with DMAD [64]. Finally, this reaction was tested extensively with various acetylenes 42 [65].

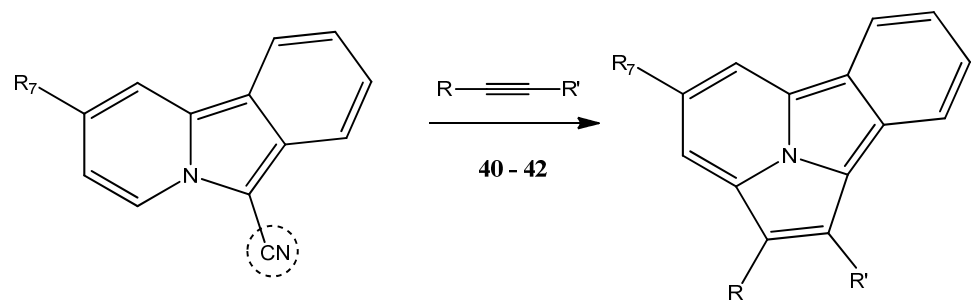

Scheme 18. Cycloaddition to 3-CN-substituted benzoindolizines (see Table 9). 
Table 9. Substituents, conditions and yields in cycloaddition of acetylene to 3-CN-substituted benzoindolizines giving benzocyclazines (Scheme 18).

\begin{tabular}{cccccc}
\hline $\mathbf{N}$ & $\mathbf{R} / \mathbf{R}^{\prime}$ & $\mathbf{R 7}$ & Cat/Solv/T $/$ Time & Yield \% & Ref. \\
\hline $\mathbf{4 0 a}$ & $\mathrm{COPh} / \mathrm{COPh}$ & $\mathrm{H}$ & $\mathrm{Pd}-\mathrm{C} / \mathrm{PhH} / \mathrm{rt} / 46 \mathrm{~h}$ & 82 & {$[62,63]$} \\
$\mathbf{4 0 b}$ & $\mathrm{COPh} / \mathrm{COPh}$ & $\mathrm{Me}$ & $\mathrm{Pd}-\mathrm{C} / \mathrm{PhH} / \mathrm{rt} / 24 \mathrm{~h}$ & 82 & {$[62]$} \\
$\mathbf{4 0 c}$ & $\mathrm{COPh} / \mathrm{COPh}$ & $\mathrm{Ph}$ & $\mathrm{Pd}-\mathrm{C} / \mathrm{PhH} / \mathrm{rt} / 24 \mathrm{~h}$ & 69 & {$[62]$} \\
$\mathbf{4 0 d}$ & $\mathrm{COPh} / \mathrm{COPh}$ & $\mathrm{COPh}$ & $\mathrm{Pd}-\mathrm{C} / \mathrm{PhH} / \Delta / 5.5 \mathrm{~h}$ & 59 & {$[62]$} \\
$\mathbf{4 1}$ & $\mathrm{E} / \mathrm{E}$ & $\mathrm{H}$ & $\mathrm{Pd}-\mathrm{C} / \mathrm{PhMe} / \Delta / 20 \mathrm{~h}$ & 54 & {$[64]$} \\
$\mathbf{4 2 a}$ & $\mathrm{E} / \mathrm{E}$ & $\mathrm{H}$ & $\mathrm{Pd}-\mathrm{C} / \mathrm{PhMe} / \Delta / 2 \mathrm{~h}$ & 13 & {$[65]$} \\
$\mathbf{4 2 b}$ & $\mathrm{E}^{\prime \prime} / \mathrm{E}^{\prime \prime} \mathrm{a}$ & $\mathrm{H}$ & $\mathrm{Pd}-\mathrm{C} / \mathrm{PhMe} / \Delta / 20 \mathrm{~h}$ & $14\left(\mathrm{R}^{\prime}=\mathrm{H}\right)$ & {$[65]$} \\
$\mathbf{4 2 c}$ & $\mathrm{Ac} / \mathrm{Ac}$ & $\mathrm{H}$ & $\mathrm{Pd}-\mathrm{C} / \mathrm{PhMe} / \Delta / 20 \mathrm{~h}$ & 8 & {$[65]$} \\
$\mathbf{4 2 d}$ & $\mathrm{E} / \mathrm{H}$ & $\mathrm{H}$ & $\mathrm{Pd}-\mathrm{C} / \mathrm{PhMe} / \Delta / 34 \mathrm{~h}$ & $\mathrm{NG}$ & {$[65]$} \\
$\mathbf{4 2 e}$ & $\mathrm{E} / \mathrm{SiMe}$ & $\mathrm{H}$ & $\mathrm{Pd}-\mathrm{C} / \mathrm{PhMe} / \Delta / 72 \mathrm{~h}$ & $11: 14^{\mathrm{b}}$ & {$[65]$} \\
$\mathbf{4 2 f}$ & $\mathrm{E} / \mathrm{Ph}$ & $\mathrm{H}$ & $\mathrm{Pd}-\mathrm{C} / \mathrm{PhMe} / \Delta / 72 \mathrm{~h}$ & $52: 6^{\mathrm{b}}$ & {$[65]$} \\
$\mathbf{4 2 g}$ & $\mathrm{Ac} / \mathrm{Ph}$ & $\mathrm{H}$ & $\mathrm{Pd}-\mathrm{C} / \mathrm{PhMe} / \Delta / 72 \mathrm{~h}$ & $48: 7^{\mathrm{b}}$ & {$[65]$} \\
\hline
\end{tabular}

a: $\mathrm{E}^{\prime \prime}-\mathrm{CO}_{2} \mathrm{tBu} ;$; : regioisomers.

\section{Cycloaddition to Benzoindolizines: Synthesis of Benzo Derivatives of Cyclazines}

Cycloaddition of benzyne (generated differently) to indolizine $\mathbf{4 3}$ is the simplest route to benzo derivatives of cyclazine [3], Scheme 19, Table 10. The resulting structures are strongly fluorescent.<smiles>[R]c1cn2c([R])c([R])c([R])c2c([R])c1[2H]</smiles><smiles></smiles>

Scheme 19. $\mathrm{CsF}, \mathrm{MeCN}, 90^{\circ}$. A-ortho-substituted benzene with $\mathrm{SiMe}_{3}$ and $\mathrm{OSO}_{2} \mathrm{CF}_{3} ; \mathrm{B}-1$ Aminobenzotriazole $/ \mathrm{Pb}(\mathrm{OAc})_{4}$.

Table 10. Substituents and yields of cycloaddition of benzynes to indolizines (Scheme 19).

\begin{tabular}{|c|c|c|c|c|c|c|c|c|c|c|}
\hline $\mathbf{N}$ & Benzyne & $\mathbf{R} 1$ & $\mathbf{R} 2$ & R3 & R5 & R6 & R7 & R8 & Yield, \% & Ref. \\
\hline $43 a$ & $\mathrm{~A}$ & $\mathrm{H}$ & $\mathrm{Ph}$ & $\mathrm{H}$ & $\mathrm{H}$ & $\mathrm{H}$ & $\mathrm{H}$ & $\mathrm{H}$ & 23 & [3] \\
\hline $43 b$ & A & $\mathrm{Me}$ & $\mathrm{Ph}$ & $\mathrm{H}$ & $\mathrm{H}$ & $\mathrm{H}$ & $\mathrm{H}$ & $\mathrm{H}$ & 49 & [3] \\
\hline $43 c$ & A & $\mathrm{H}$ & $\mathrm{Ph}$ & $\mathrm{H}$ & $\mathrm{H}$ & $\mathrm{H}$ & $\mathrm{H}$ & $\mathrm{Me}$ & 44 & [3] \\
\hline $43 d$ & A & $\mathrm{H}$ & $\mathrm{Ph}$ & $\mathrm{H}$ & $\mathrm{H}$ & $\mathrm{H}$ & $\mathrm{Me}$ & $\mathrm{H}$ & 55 & [3] \\
\hline $43 e$ & A & $\mathrm{CN}$ & $\mathrm{H}$ & $\mathrm{H}$ & $\mathrm{H}$ & $\mathrm{H}$ & $\mathrm{Me}$ & $\mathrm{H}$ & 51 & [3] \\
\hline $43 f$ & A & $\mathrm{E}$ & $\mathrm{H}$ & $\mathrm{H}$ & $\mathrm{H}$ & $\mathrm{H}$ & $\mathrm{H}$ & $\mathrm{H}$ & 50 & [3] \\
\hline $43 g$ & A & E & E & $\mathrm{H}$ & $\mathrm{H}$ & $\mathrm{H}$ & $\mathrm{H}$ & $\mathrm{H}$ & 50 & [3] \\
\hline $43 h$ & A & $E^{\prime}$ & $\mathrm{E}^{\prime}$ & $\mathrm{H}$ & $\mathrm{H}$ & $\mathrm{H}$ & $\mathrm{H}$ & $\mathrm{H}$ & 37 & [3] \\
\hline $43 i$ & A & $E^{\prime}$ & $E^{\prime}$ & $\mathrm{H}$ & $\mathrm{H}$ & $\mathrm{H}$ & $\mathrm{Me}$ & $\mathrm{H}$ & 30 & [3] \\
\hline $43 j$ & A & $\mathrm{E}$ & $\mathrm{E}$ & $\mathrm{CN}$ & $\mathrm{H}$ & $\mathrm{Me}$ & $\mathrm{H}$ & $\mathrm{H}$ & 75 & [3] \\
\hline $43 k$ & A & $\mathrm{COPh}$ & $\mathrm{Ph}$ & $\mathrm{H}$ & $\mathrm{H}$ & $\mathrm{H}$ & $\mathrm{Me}$ & $\mathrm{H}$ & 51 & [3] \\
\hline 431 & B & $\mathrm{Me}$ & $\mathrm{Ph}$ & $\mathrm{H}$ & $\mathrm{H}$ & $\mathrm{H}$ & $\mathrm{H}$ & $\mathrm{H}$ & 40 & [3] \\
\hline $43 m$ & B & $\mathrm{H}$ & $\mathrm{Ph}$ & $\mathrm{H}$ & $\mathrm{H}$ & $\mathrm{H}$ & $\mathrm{H}$ & $\mathrm{Me}$ & 18 & [3] \\
\hline $43 m$ & B & $\mathrm{H}$ & $\mathrm{Ph}$ & $\mathrm{H}$ & $\mathrm{H}$ & $\mathrm{H}$ & $\mathrm{Me}$ & $\mathrm{H}$ & 42 & [3] \\
\hline 430 & A & $\mathrm{E}$ & $\mathrm{H}$ & $\mathrm{H}$ & $\mathrm{H}$ & $\mathrm{H}$ & \multicolumn{2}{|c|}{ 7,8-Benzo } & 60 & [3] \\
\hline $43 p$ & A & $\mathrm{CN}$ & $\mathrm{H}$ & $\mathrm{H}$ & $\mathrm{H}$ & $\mathrm{H}$ & \multicolumn{2}{|c|}{ 7,8-Benzo } & 52 & [3] \\
\hline $43 q$ & A & $E^{\prime}$ & $E^{\prime}$ & $\mathrm{H}$ & $\mathrm{H}$ & $\mathrm{H}$ & \multicolumn{2}{|c|}{ 7,8-Benzo } & 58 & [3] \\
\hline
\end{tabular}

Condensed structures from 43r,s with coumarin ring were similarly obtained, Scheme 20 [3]. 


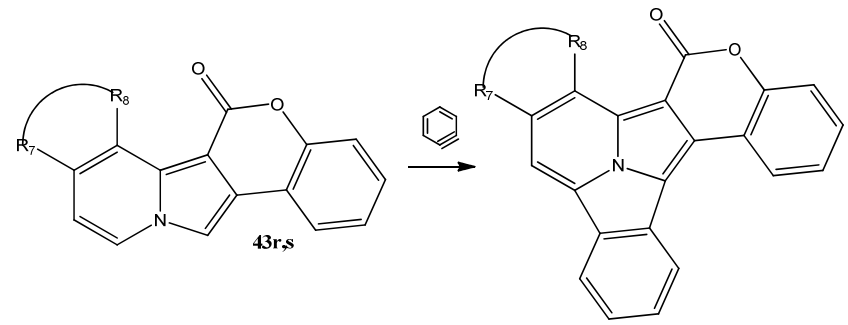

Scheme 20. CsF, MeCN, 90 . 43r (R7R8 = H, 93\%), 43s (R7,R8 = benzo, 62\%), Method A.

Another route to the same benzo-skeleton is cycloaddition of alkynes to benzo[a]indoli zines. This reaction was studied with acetylenes containing boron substituents, alone 44a-c [37] or together with nitrogen-containing heterocycle on another end of acetylene 45a-e [5], Scheme 21, Table 11. In one experiment 46 benzyne was generated from $\mathrm{PhBr}$; this resulted in dibenzocyclazine was obtained with low yield [66].<smiles>c1ccc2c(c1)cn1ccccc21</smiles>
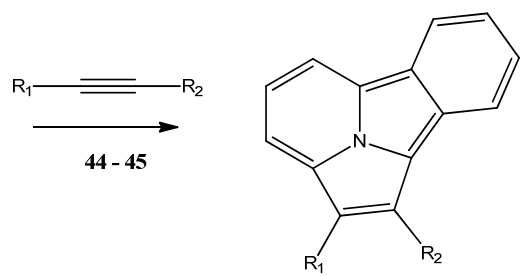

Scheme 21. Cycloaddition to benzoindolizines see Table 11).

Table 11. Substituents and yields (ratio of isomers) of cycloaddition to benzoindolizines (Scheme 21).

\begin{tabular}{|c|c|c|c|c|c|}
\hline $\mathbf{N}$ & R1 & R2 & Solv/T ${ }^{\circ} /$ Time$/$ Oxidant & Yield, \% & Ref. \\
\hline $44 a$ & $\mathrm{Mes}_{2} \mathrm{~B}$ & $\mathrm{H}$ & $\mathrm{PhMe} / \mathrm{rt} / 30 \mathrm{~m} / \mathrm{DDQ}$ & 89 & [37] \\
\hline $44 \mathrm{~b}$ & $2-\left(\mathrm{Mes}_{2} \mathrm{~B}\right) \mathrm{Ph}$ & $\mathrm{H}$ & $\mathrm{PhMe} / \Delta / 3 \mathrm{~d} / \mathrm{DDQ}$ & 75 & [37] \\
\hline $44 c$ & $4-\left(\mathrm{Mes}_{2} \mathrm{~B}\right) \mathrm{Ph}$ & $\mathrm{H}$ & $\mathrm{PhMe} / \Delta / 3 \mathrm{~d} / \mathrm{DDQ}$ & 83 & [37] \\
\hline $45 \mathbf{a}$ & 4-( $\left.\mathrm{Mes}_{2} \mathrm{~B}\right) \mathrm{Ph}$ & 2-Py & (1) $\mathrm{PhMe} / 120^{\circ} \mathrm{C} / 5-6 \mathrm{~d} ;$ (2) DDQ $/ \mathrm{rt} / 0.5 \mathrm{~h}$ & $79: 2$ & [5] \\
\hline $45 \mathrm{~b}$ & $2-\left(\mathrm{Mes}_{2} \mathrm{~B}\right) \mathrm{Ph}$ & 2-Py & (1) $\mathrm{PhMe} / 120^{\circ} \mathrm{C} / 5-6 \mathrm{~d}$; (2) DDQ/rt/0.5 h & $82: 4$ & [5] \\
\hline $45 c$ & $2-\left(\mathrm{Mes}_{2} \mathrm{~B}\right) \mathrm{Ph}$ & 2-Isoquinolyl & (1) $\mathrm{PhMe} / 120^{\circ} \mathrm{C} / 5-6 \mathrm{~d}$; (2) DDQ $/ \mathrm{rt} / 0.5 \mathrm{~h}$ & $72: 18$ & [5] \\
\hline $45 \mathrm{~d}$ & $2-\left(\mathrm{Mes}_{2} \mathrm{~B}\right) \mathrm{Ph}$ & 2-Benzotiazolyl & (1) $\mathrm{PhMe} / 120^{\circ} \mathrm{C} / 5-6 \mathrm{~d}$; (2) DDQ/rt/0.5 h & $62: 19$ & [5] \\
\hline $45 e$ & $2-\left(\mathrm{Mes}_{2} \mathrm{~B}\right) \mathrm{Ph}$ & 2-Tiazolyl & (1) $\mathrm{PhMe} / 120^{\circ} \mathrm{C} / 5-6 \mathrm{~d} ;(2) \mathrm{DDQ} / \mathrm{rt} / 0.5 \mathrm{~h}$ & 68:14 & [5] \\
\hline 46 & \multicolumn{2}{|c|}{ Benzo } & $\begin{array}{c}\text { 2,2,6,6-tetramethylpiperidine, } \mathrm{n} \text {-BuLi, } \\
\mathrm{PhBr} / \mathrm{THF} /-78^{\circ} \mathrm{C} / 1 \mathrm{~h}\end{array}$ & 6.6 & [66] \\
\hline
\end{tabular}

Tominaga showed that indolizines $47 \mathbf{a}, \mathbf{b}$ having annelated benzene ring across the bond C7-C8 underwent [8+2] cycloaddition forming benzo[g]cycl[3.2.2]azines [29,67], Scheme 22.
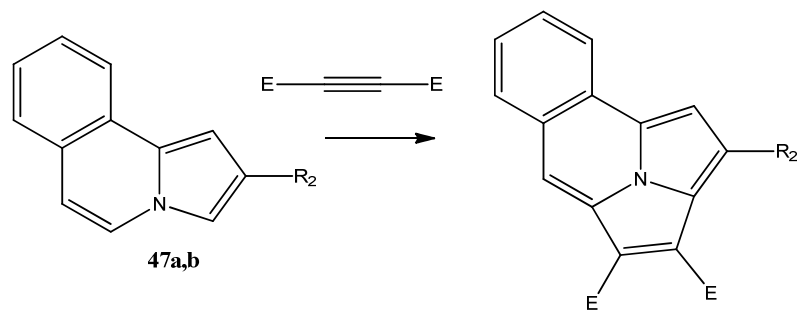

Scheme 22. Pd-C/PhMe/ $\Delta / 30 h .47 a R^{2}=H(33 \%), 47 b R^{2}=\operatorname{MeS}(27 \%)$.

In another paper [68], he demonstrated a similar reaction of dibenzoindolizine 48 with DMAD leading to dibenzo[a,h]cycl[3.2.2]azine, Scheme 23. 

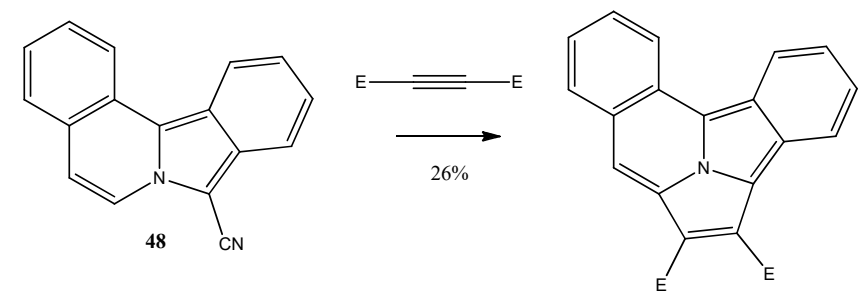

Scheme 23. $\mathrm{MePh}+\mathrm{HOAc} / \Delta / 20 \mathrm{~h}$.

Isomeric indolizines $49 \mathbf{a}, \mathbf{b}$ annelated across the bond $\mathrm{C} 6-\mathrm{C} 7$ with benzothiophene underwent cycloaddition with DEAD (PhMe/ $\Delta / 6 \mathrm{~h}$ ) without any catalyst [69], Scheme 24.

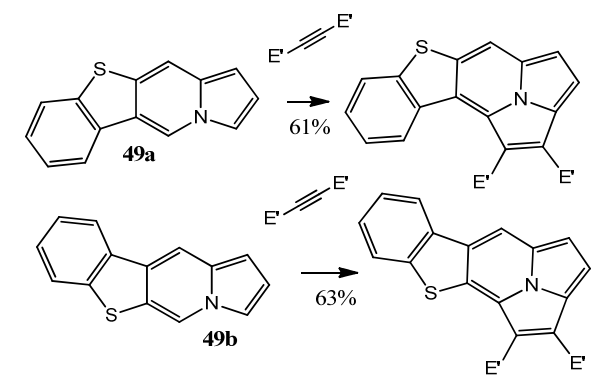

Scheme 24. Example of cycloaddition to fused indolizines.

The last example is 1,2,5,6-dibenzocycl[2,2,3]azine obtained with a yield of $54 \%$ from dibenzoindolizine and DEAD in presence of Pd-C [70], Scheme 25.<smiles>c1ccc2cn3cc4ccccc4c3cc2c1</smiles>

50

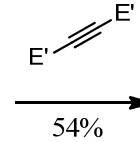

$54 \%$<smiles></smiles>

Scheme 25. $\mathrm{Pd}-\mathrm{C} / \mathrm{PhMe} / \Delta / 14 \mathrm{~h}$.

This reaction is featured, firstly, because it was the first cycloaddition in the history of indolizines that even made an influence on Boekelheide. Second, is that the structure of dibenzoindolizine is extremely polyenic (annelation in indolizine appears across two single bonds), and therefore, the process could be better treated as [2+16] rather than $[2+8]$ cycloaddition.

\section{Cycloadditions Where Indolizines Are Intermediates}

There are many examples of cyclazine synthesis where the intermediates are indolizines. First, there are so-called 3 component reactions: picoline and bromoketone in the presence of a base (Chichibabin combination to obtain indolizine) and alkyne. Two examples of such combination were reported in microwave conditions [71,72], Scheme 26, Table 12. 


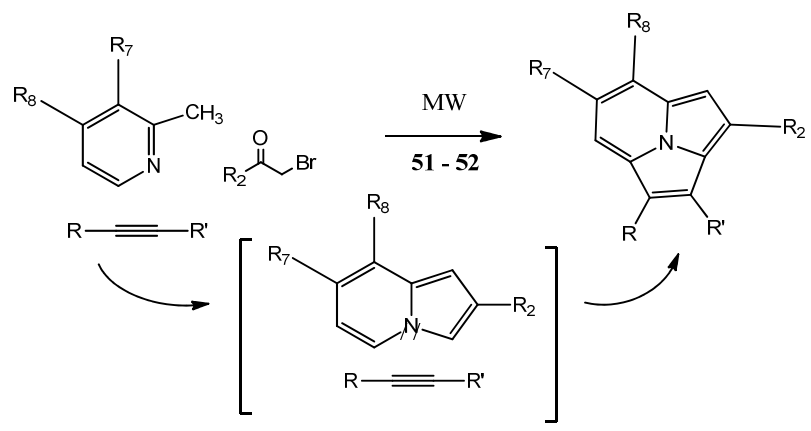

Scheme 26. Microwave three-component synthesis of cyclazines (see Table 12).

Table 12. Substituents and yields of microwave three-component synthesis of cyclazines (Scheme 26).

\begin{tabular}{|c|c|c|c|c|c|c|c|}
\hline $\mathbf{N}$ & $\mathbf{R}$ & $\mathbf{R}^{\prime}$ & $\mathbf{R} 2$ & R7 & R8 & Yield, \% & Ref. \\
\hline $51 a$ & $E$ & $\mathrm{E}$ & $\mathrm{Ph}$ & $\mathrm{H}$ & $\mathrm{H}$ & 90 & {$[71]^{\mathrm{a}}$} \\
\hline $51 b$ & $\mathrm{E}$ & $\mathrm{E}$ & 4-Me-Ph & $\mathrm{H}$ & $\mathrm{H}$ & 92 & [71] \\
\hline $51 c$ & $\mathrm{E}$ & $\mathrm{E}$ & 4-Cl-Ph & $\mathrm{H}$ & $\mathrm{H}$ & 60 & [71] \\
\hline $51 d$ & $\mathrm{E}$ & $\mathrm{E}$ & 4-NO2-Ph & $\mathrm{H}$ & $\mathrm{H}$ & 20 & [71] \\
\hline $51 \mathrm{e}$ & $\mathrm{E}$ & $\mathrm{E}$ & Polycyclic R & $\mathrm{H}$ & $\mathrm{H}$ & 78 & [71] \\
\hline $51 f$ & $\mathrm{E}$ & $\mathrm{E}$ & 1-Cyclohexenyl & $\mathrm{H}$ & $\mathrm{H}$ & 74 & [71] \\
\hline $51 \mathrm{~g} *$ & $\mathrm{H}$ & $E^{\prime}$ & $\mathrm{Ph}$ & $\mathrm{H}$ & $\mathrm{H}$ & 78 & [71] \\
\hline $51 h *$ & $\mathrm{H}$ & $E^{\prime}$ & $\mathrm{Ph}$ & $\mathrm{Me}$ & $\mathrm{H}$ & 80 & [71] \\
\hline $51 i *$ & $\mathrm{H}$ & $E^{\prime}$ & 4-Me-Ph & $\mathrm{Me}$ & $\mathrm{H}$ & 74 & [71] \\
\hline $51 j *$ & $\mathrm{H}$ & $E^{\prime}$ & 4-Cl-Ph & $\mathrm{H}$ & $\mathrm{Me}$ & 65 & [71] \\
\hline $51 k *$ & $\mathrm{H}$ & $E^{\prime}$ & 4-NO2-Ph & $\mathrm{H}$ & $\mathrm{Me}$ & 22 & [71] \\
\hline $52 a$ & $\mathrm{E}$ & $\mathrm{E}$ & $\mathrm{Ph}$ & $\mathrm{H}$ & $\mathrm{H}$ & 37 & {$[72]^{b}$} \\
\hline $52 b$ & $\mathrm{E}$ & $\mathrm{E}$ & $4-\mathrm{NO}_{2} \mathrm{Ph}$ & $\mathrm{H}$ & $\mathrm{H}$ & 78 & [72] \\
\hline $52 \mathrm{c}$ & $\mathrm{E}$ & $\mathrm{E}$ & 4-ClPh & $\mathrm{H}$ & $\mathrm{H}$ & 46 & [72] \\
\hline $52 d$ & $\mathrm{E}$ & $\mathrm{E}$ & 4-MeOPh & $\mathrm{H}$ & $\mathrm{H}$ & 23 & [72] \\
\hline $52 e$ & $\mathrm{E}$ & $\mathrm{E}$ & 4-MePh & $\mathrm{H}$ & $\mathrm{H}$ & 39 & [72] \\
\hline $52 f$ & $\mathrm{E}$ & $\mathrm{E}$ & 4-OHPh & $\mathrm{H}$ & $\mathrm{H}$ & 36 & [72] \\
\hline $52 \mathrm{~g}$ & $\mathrm{E}$ & $\mathrm{E}$ & 4-BrPh & $\mathrm{H}$ & $\mathrm{H}$ & 49 & [72] \\
\hline $52 \mathrm{~h}$ & $\mathrm{E}$ & $\mathrm{E}$ & $4-\mathrm{FPh}$ & $\mathrm{H}$ & $\mathrm{H}$ & 42 & [72] \\
\hline
\end{tabular}

a: $\mathrm{K}_{2} \mathrm{CO}_{3}$, water MW, $100{ }^{\circ} \mathrm{C} 2-5 \mathrm{~min} ;{ }^{\mathrm{b}}$ : Alumina, $300 \mathrm{~W}, 2 \mathrm{~min} ;{ }^{*}$ Attention: unexpected products, place of $\mathrm{R}$ and $\mathrm{R}^{\prime}$ groups should be reversed. Probably mistake made by the authors.

Another example is given by cycloaddition to pyridone 53a giving cyclazine 53b [73] Scheme 27. Evidently, intermediates are (partially isolated) indolizine 55e which is obtained by sequence $55 \mathrm{c}-55 \mathrm{~d}$.

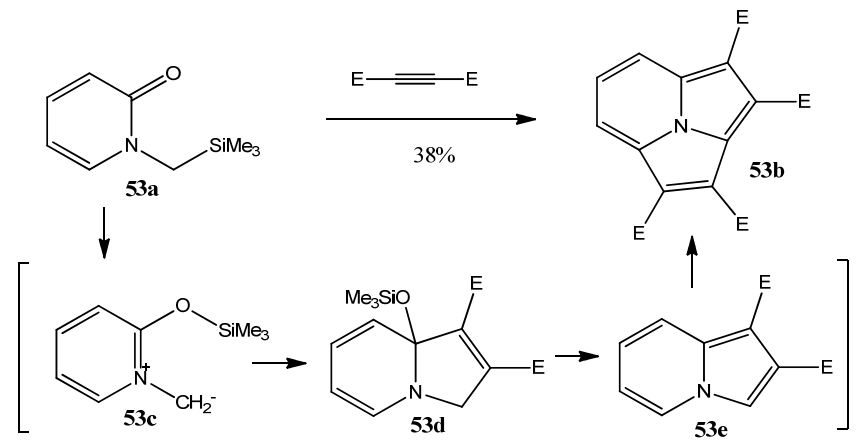

Scheme 27. $\mathrm{PhMe} / \Delta / 30 \mathrm{~h}$.

Another example of cyclazine $\mathbf{5 4 b}$ synthesis from pyridine 54a with ethyl propiolate via indolizine 54c [74] is illustrated in Scheme 28. Indolizine 54c could be isolated. 


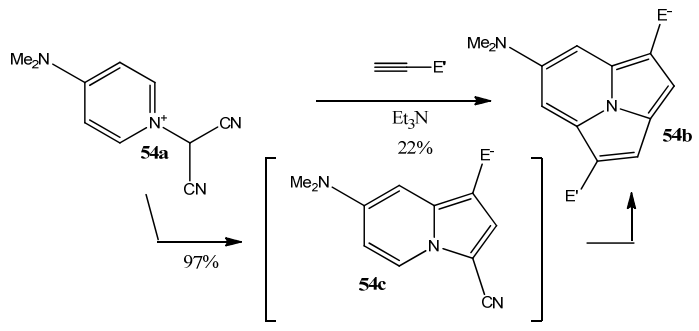

Scheme 28. TEA (1.2 eq), ethyl propiolate (1.5 eq), $\mathrm{CH}_{3} \mathrm{CN}+\mathrm{DMF}, \mathrm{rt}, 24 \mathrm{~h}$.

A similar reaction is between the same pyridine and benzyne [75-77] forming dibenzoindolizine, Scheme 29, Table 13.

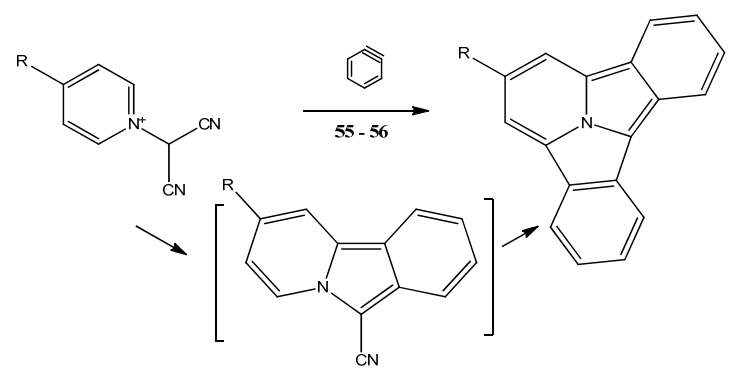

Scheme 29. Synthesis of benzocyclazines from pyridinium ylides (Table 13).

Table 13. Substituents and yields of benzocyclazines from pyridinium ylide (Scheme 29).

\begin{tabular}{cccccc}
\hline $\mathbf{N}$ & $\mathbf{R}$ & $\mathbf{A}$, Yield, $\%$ & B, Yield, $\%$ & C, Yield, $\%$ & Ref. \\
\hline $\mathbf{5 5 a}$ & $\mathrm{H}$ & $11(20)$ & $4(35)$ & 39 & {$[76]$} \\
$\mathbf{5 5 b}$ & $\mathrm{Me}$ & $5(22)$ & $2(12)$ & 33 & {$[76]$} \\
$\mathbf{5 5} \mathbf{c}$ & $\mathrm{Ph}$ & $3(44)$ & $2(10)$ & 38 & {$[76]$} \\
$\mathbf{5 5 d}$ & $\mathrm{PhCO}$ & $0.5(21)$ & $17(24)$ & 25 & {$[76]$} \\
$\mathbf{5 5} \mathbf{5}$ & $\mathrm{MeOCO}$ & $3(33)$ & 32 & - & {$[76]$} \\
$\mathbf{5 5 f}$ & $\mathrm{MeCO}$ & Trace $(27)$ & 18 & - & {$[76]$} \\
$\mathbf{5 6 a}$ & $1,2-\mathrm{Me}$ & 12 & - & - & {$[77]$} \\
$\mathbf{5 6 b}$ & $1,3-\mathrm{Me}$ & 5 & - & - & {$[77]$} \\
\hline
\end{tabular}

In brackets-yield of benzoindolizine. A-diphenyliodonium-2-carboxylate monohydrate $200^{\circ} \mathrm{C}$; B - anthranilic acid and isopentyl nitrite in refluxing chloroform-acetone; $\mathrm{C}-6$-cyanobenzo[a]indolizine diphenyliodonium-2-carboxylatem monohydrate in DME $200{ }^{\circ} \mathrm{C} 3 \mathrm{~h}$.

Interesting multistep reaction starting from pyridine $57 \mathbf{a}$ and finishing with cyclazine 57b with the yields $15-70 \%$ was observed independently by Acheson and Pohjala [51,78-82], Scheme 30. The mechanism of this process included Perkin reaction and intermediate formation of indolizine skeleton $57 \mathrm{c}$.

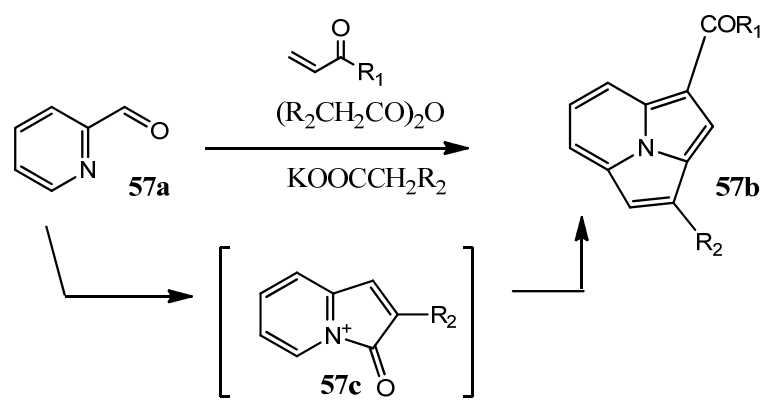

Scheme 30. Multistep reaction ending with cyclazines. 


\section{Cycloaddition to Azacyclazines and Their Benzo-Derivatives}

The first cycloaddition to aza-analogs of indolizine was observed by Boekelheide [83] in the reaction of imidazo[1,2-a]pyridine 58 with DMAD in presence of Pd-C, Scheme 31 and Table 14. It was also shown that 6-azaindolizine 59 [84] (but not 7-aza-derivative [25]) can be involved in a similar process. Soon it was proved also for 8-aza-indolizine 60a and its 7-oxo-analog $61 \mathrm{~b}$ [85]. 1-Azaindolizine bearing 2-SO $\mathrm{S}_{2} \mathrm{Me}$ group failed to go in such cycloaddition [86], whereas the same structures with 2-SMe group $\mathbf{6 1 a , b}$ [87] and their [h]-benzannelated derivatives 62 [88] formed the desired azacyclazines with DMAD. In our recent work, we proved that MAC could react with 1-azaindolizine 63 giving azacyclazine in the open air [36]. Diphenylacetylene was capable to transform imidazopyridine 64 to azacyclazine under the action of $\mathrm{Pd}(\mathrm{OAc})_{2} / \mathrm{Cu}(\mathrm{OAc})_{2}[89]$.

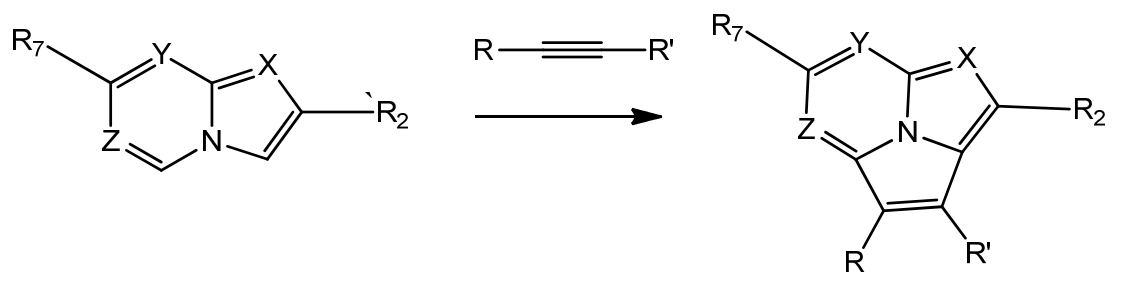

Scheme 31. Synthesis of azacyclazines from azaindolizines (Table 14).

Table 14. Substituents, conditions and yields of azacyclazines from azaindolizines (Scheme 31).

\begin{tabular}{|c|c|c|c|c|c|c|c|c|c|}
\hline $\mathbf{N}$ & $\mathbf{R} / \mathbf{R}^{\prime}$ & $x$ & $\mathbf{R} 2$ & $\mathbf{Z}$ & R7 & $\mathbf{Y}$ & Cat/Solv/T ${ }^{\circ} /$ Time & Yield, \% & Ref. \\
\hline 58 & $\mathrm{E} / \mathrm{E}$ & $\mathrm{N}$ & $\mathrm{Ph}$ & $\mathrm{H}$ & $\mathrm{H}$ & $\mathrm{H}$ & $\mathrm{Pd}-\mathrm{C} / \mathrm{MePh} / \Delta / 25 \mathrm{~h}$ & 29 & [83] \\
\hline 59 & $\mathrm{E} / \mathrm{E}$ & $\mathrm{H}$ & $\mathrm{Ph}$ & $\mathrm{N}$ & $\mathrm{Me}$ & $\mathrm{H}$ & $\mathrm{Pd}-\mathrm{C} / \mathrm{MePh} / \Delta / 21 \mathrm{~h}$ & 28 & [84] \\
\hline $60 a$ & $\mathrm{E} / \mathrm{E}$ & $\mathrm{H}$ & $\mathrm{Me}$ & $\mathrm{H}$ & $\mathrm{Me}$ & $\mathrm{N}$ & $\mathrm{Pd}-\mathrm{C} / \mathrm{MePh} / \Delta /$ & 66 & [85] \\
\hline $60 b$ & $\mathrm{E} / \mathrm{E}$ & $\mathrm{H}$ & $\mathrm{Me}$ & $\mathrm{H}$ & $\mathrm{O}=$ & $\mathrm{NMe}$ & $\mathrm{Pd}-\mathrm{C} / \mathrm{MePh} / \Delta /$ & 59 & [85] \\
\hline 61 & $\mathrm{E} / \mathrm{E}$ & $\mathrm{N}$ & $\mathrm{SMe}$ & $\mathrm{H}$ & \multicolumn{2}{|c|}{$(\mathrm{CH}=\mathrm{CH})_{2}$} & $\mathrm{Pd}-\mathrm{C} / \mathrm{MePh} / \Delta / 30 \mathrm{~h}$ & 6 & [88] \\
\hline $62 a$ & $\mathrm{E} / \mathrm{E}$ & $\mathrm{N}$ & $\mathrm{SMe}$ & $\mathrm{H}$ & $\mathrm{H}$ & $\mathrm{H}$ & $\mathrm{Pd}-\mathrm{C} / \mathrm{MePh} / \Delta / 30 \mathrm{~h}$ & 36 & [87] \\
\hline $62 b$ & $\mathrm{E} / \mathrm{E}$ & $\mathrm{N}$ & $\mathrm{SMe}$ & $\mathrm{MeC}$ & $\mathrm{H}$ & $\mathrm{MeC}$ & $\mathrm{Pd}-\mathrm{C} / \mathrm{MePh} / \Delta / 30 \mathrm{~h}$ & 40 & [87] \\
\hline 63 & $\mathrm{E} / \mathrm{H}$ & $\mathrm{N}$ & $\mathrm{t}-\mathrm{Bu}$ & $\mathrm{H}$ & $\mathrm{H}$ & $\mathrm{H}$ & $\mathrm{O}_{2} / \mathrm{MePh} / \Delta / 4 \mathrm{~h}$ & 75 & [36] \\
\hline 64 & $\mathrm{Ph} / \mathrm{Ph}$ & $\mathrm{N}$ & $\mathrm{H}$ & $\mathrm{H}$ & $\mathrm{H}$ & $\mathrm{H}$ & $\begin{array}{c}\mathrm{Pd}(\mathrm{OAc})_{2} / \mathrm{Cu}(\mathrm{OAc})_{2} \\
\mathrm{LiOAc} / \mathrm{DMAc} / 120^{\circ} / 8 \mathrm{~h}\end{array}$ & 49 & [89] \\
\hline
\end{tabular}

Mesoionic structure 65a underwent cycloaddition with DMAD giving fully covalent structure $65 \mathbf{b}$ proved by $\mathrm{X}$-ray [55], Scheme 32 .<smiles>CN1C(=O)c2c(F)c(F)c3cccc(n23)N1CC(F)(F)F</smiles>

Scheme 32. Synthesis of azacyclazinone from mesoionic structure.

Imidazopyridines 66 are transformed to azacyclazines under the action of $\mathrm{Pd}(\mathrm{OAc})_{2} /$ $\mathrm{Cu}(\mathrm{OAc})_{2}$ [90] and [91], Scheme 33, Table 15. 


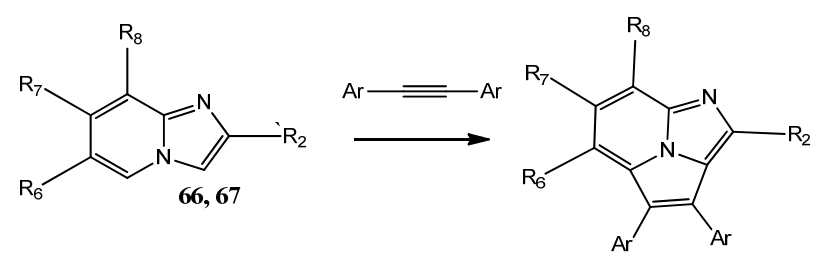

Scheme 33. $\mathrm{Pd}(\mathrm{OAc})_{2}, \mathrm{Cu}(\mathrm{OAc})_{2}, \mathrm{DMSO}, 110{ }^{\circ} \mathrm{C}, 12 \mathrm{~h}$ [90]. Pd-Se complex $\mathrm{Cu}(\mathrm{OAc})_{2}, \mathrm{KOtBu}$, DMAC, $120^{\circ} \mathrm{C}, 16 \mathrm{~h}$ [91].

Table 15. Substituents and yields of reaction of 2-arylimidazopiridines and diarylacetylenes (Scheme 33).

\begin{tabular}{|c|c|c|c|c|c|c|c|}
\hline $\mathbf{N}$ & Ar & $\mathbf{R} 2$ & R6 & R7 & R8 & Yield, \% & Ref. \\
\hline $66 a$ & $\mathrm{Ph}$ & $\mathrm{Ph}$ & $\mathrm{H}$ & $\mathrm{H}$ & $\mathrm{H}$ & 78 & [90] \\
\hline $66 b$ & $\mathrm{Ph}$ & $\mathrm{Ph}$ & $\mathrm{H}$ & $\mathrm{H}$ & $\mathrm{Me}$ & 76 & [90] \\
\hline $66 c$ & $\mathrm{Ph}$ & $\mathrm{Ph}$ & $\mathrm{H}$ & $\mathrm{Me}$ & $\mathrm{H}$ & 75 & [90] \\
\hline $66 d$ & $\mathrm{Ph}$ & $\mathrm{Ph}$ & $\mathrm{Cl}$ & $\mathrm{H}$ & $\mathrm{H}$ & 68 & [90] \\
\hline $66 \mathrm{e}$ & $\mathrm{Ph}$ & 4-MePh & $\mathrm{H}$ & $\mathrm{H}$ & $\mathrm{H}$ & 79 & [90] \\
\hline $66 f$ & $\mathrm{Ph}$ & 4-FPh & $\mathrm{H}$ & $\mathrm{H}$ & $\mathrm{H}$ & 75 & [90] \\
\hline $66 \mathrm{~g}$ & $\mathrm{Ph}$ & 4-ClPh & $\mathrm{H}$ & $\mathrm{H}$ & $\mathrm{Me}$ & 73 & [90] \\
\hline $66 h$ & $\mathrm{Ph}$ & 4-CNPh & $\mathrm{H}$ & $\mathrm{H}$ & $\mathrm{H}$ & 71 & [90] \\
\hline $66 \mathbf{i}$ & $\mathrm{Ph}$ & $4-\mathrm{NO}_{2} \mathrm{Ph}$ & $\mathrm{H}$ & $\mathrm{H}$ & $\mathrm{H}$ & 67 & [90] \\
\hline $66 \mathbf{j}$ & $\mathrm{Ph}$ & $\mathrm{CF}_{3}$ & $\mathrm{H}$ & $\mathrm{H}$ & $\mathrm{H}$ & 64 & [90] \\
\hline $66 \mathrm{k}$ & $\mathrm{Ph}$ & $\mathrm{i}-\mathrm{Bu}$ & $\mathrm{H}$ & $\mathrm{H}$ & $\mathrm{H}$ & 63 & [90] \\
\hline 661 & 4-MePh & $\mathrm{Ph}$ & $\mathrm{H}$ & $\mathrm{H}$ & $\mathrm{H}$ & 77 & [90] \\
\hline $66 \mathrm{~m}$ & 4-MePh & $\mathrm{Ph}$ & $\mathrm{H}$ & $\mathrm{Me}$ & $\mathrm{H}$ & 74 & [90] \\
\hline $66 n$ & 4-MeOPh & $\mathrm{Ph}$ & $\mathrm{H}$ & $\mathrm{H}$ & $\mathrm{H}$ & 70 & [90] \\
\hline 660 & 4-MeOPh & $\mathrm{Ph}$ & $\mathrm{H}$ & $\mathrm{Me}$ & $\mathrm{H}$ & 69 & [90] \\
\hline $66 p$ & 4-MeOPh & $\mathrm{i}-\mathrm{Bu}$ & $\mathrm{H}$ & $\mathrm{H}$ & $\mathrm{H}$ & 61 & [90] \\
\hline $67 a$ & $\mathrm{Ph}$ & $\mathrm{Ph}$ & $\mathrm{H}$ & $\mathrm{H}$ & $\mathrm{H}$ & 68 & [91] \\
\hline $67 b$ & $\mathrm{Ph}$ & 1-Naphtyl & $\mathrm{H}$ & $\mathrm{H}$ & $\mathrm{H}$ & 69 & [91] \\
\hline $67 c$ & $\mathrm{Ph}$ & $\mathrm{Ph}$ & $\mathrm{H}$ & $\mathrm{Cl}$ & $\mathrm{Cl}$ & 57 & [91] \\
\hline $67 d$ & $\mathrm{Ph}$ & $\mathrm{Ph}$ & $\mathrm{H}$ & $\mathrm{Br}$ & $\mathrm{Br}$ & 51 & [91] \\
\hline $67 e$ & $\mathrm{Ph}$ & $\mathrm{Ph}$ & $\mathrm{Me}$ & $\mathrm{H}$ & $\mathrm{H}$ & 76 & [91] \\
\hline $67 f$ & $\mathrm{Ph}$ & 4-CN-Ph & $\mathrm{H}$ & $\mathrm{H}$ & $\mathrm{H}$ & 64 & [91] \\
\hline $67 g$ & $\mathrm{Ph}$ & 4-F-Ph & $\mathrm{H}$ & $\mathrm{H}$ & $\mathrm{H}$ & 68 & [91] \\
\hline $67 \mathrm{~h}$ & $\mathrm{Ph}$ & 4-Br-Ph & $\mathrm{H}$ & $\mathrm{H}$ & $\mathrm{H}$ & 66 & [91] \\
\hline $67 \mathrm{i}$ & $\mathrm{Ph}$ & 4-MeO-Ph & $\mathrm{H}$ & $\mathrm{H}$ & $\mathrm{H}$ & 73 & [91] \\
\hline $67 j$ & $\mathrm{Ph}$ & 4-F-Ph & $\mathrm{Me}$ & $\mathrm{H}$ & $\mathrm{H}$ & 61 & [91] \\
\hline $67 \mathrm{k}$ & $\mathrm{Ph}$ & 2-Tienyl & $\mathrm{H}$ & $\mathrm{H}$ & $\mathrm{H}$ & 67 & [91] \\
\hline 671 & $\mathrm{Ph}$ & $\mathrm{Ph}$ & $\mathrm{H}$ & $\mathrm{H}$ & $\mathrm{H}$ & 63 & [91] \\
\hline $67 \mathrm{~m}$ & 4-Br-Ph & $\mathrm{Ph}$ & $\mathrm{H}$ & $\mathrm{H}$ & $\mathrm{H}$ & 78 & [91] \\
\hline $67 n$ & $E^{\prime}$ & $\mathrm{Ph}$ & $\mathrm{H}$ & $\mathrm{H}$ & $\mathrm{H}$ & 31 & [91] \\
\hline
\end{tabular}

Imidazo[1,2-a]pyridines and imidazo[1,2-a]pyrimidines readily reacted with diaryl acetylenes in presence of catalyst [92], Scheme 34, Table 16.
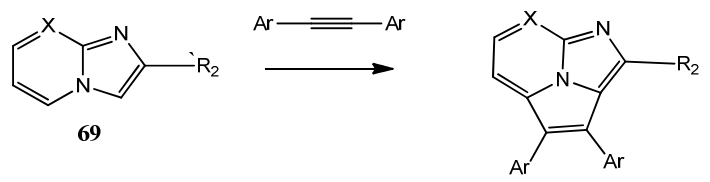

Scheme 34. Pd-NHC complex, $\mathrm{Cu}(\mathrm{OAc})_{2}, \mathrm{TBAB}, \mathrm{DMA}, 90^{\circ} \mathrm{C}, 12 \mathrm{~h}$. 
Table 16. Substituents and yields of reaction of 2-arylimidazopiridines and diarylacetylenes (Scheme 34).

\begin{tabular}{cccccc}
\hline $\mathbf{N}$ & $\mathbf{A r} / \mathrm{Ar}$ & $\mathbf{R 2}$ & $\mathbf{R}$ & Yield, \% & Ref. \\
\hline $\mathbf{6 9 a}$ & $\mathrm{Ph}$ & $\mathrm{Me}$ & $\mathrm{H}$ & 67 & {$[92]$} \\
$\mathbf{6 9 b}$ & $4-\mathrm{Me}-\mathrm{Ph}$ & $\mathrm{Me}$ & $\mathrm{H}$ & 71 & {$[92]$} \\
$\mathbf{6 9 c}$ & $2-\mathrm{Me}-\mathrm{Ph}$ & $\mathrm{Me}$ & $\mathrm{H}$ & 60 & {$[92]$} \\
$\mathbf{6 9}$ & $4-\mathrm{MeO}-\mathrm{Ph}$ & $\mathrm{Me}$ & $\mathrm{H}$ & 63 & {$[92]$} \\
$\mathbf{6 9 f}$ & $4-\mathrm{F}-\mathrm{Ph}$ & $\mathrm{Me}$ & $\mathrm{H}$ & 68 & {$[92]$} \\
$\mathbf{6 9 g}$ & $4-\mathrm{Cl}-\mathrm{Ph}$ & $\mathrm{Me}$ & $\mathrm{H}$ & 68 & {$[92]$} \\
$\mathbf{6 9 h}$ & $4-\mathrm{Br}-\mathrm{Ph}$ & $\mathrm{Me}$ & $\mathrm{H}$ & 63 & {$[92]$} \\
$\mathbf{6 9 i}$ & $\mathrm{Ph}$ & $\mathrm{t}-\mathrm{Bu}$ & $\mathrm{H}$ & 78 & {$[92]$} \\
$\mathbf{6 9 j}$ & $4-\mathrm{Me}-\mathrm{Ph}$ & $\mathrm{t}-\mathrm{Bu}$ & $\mathrm{H}$ & 75 & {$[92]$} \\
$\mathbf{6 9 k}$ & $4-\mathrm{F}-\mathrm{Ph}$ & $\mathrm{t}-\mathrm{Bu}$ & $\mathrm{H}$ & 74 & {$[92]$} \\
$\mathbf{6 9 1}$ & $4-\mathrm{Cl}-\mathrm{Ph}$ & $\mathrm{t}-\mathrm{Bu}$ & $\mathrm{H}$ & 80 & {$[92]$} \\
$\mathbf{6 9 m}$ & $4-\mathrm{Br}-\mathrm{Ph}$ & $\mathrm{t}-\mathrm{Bu}$ & $\mathrm{H}$ & 66 & {$[92]$} \\
$\mathbf{6 9 n}$ & $\mathrm{Ph}$ & $\mathrm{t}-\mathrm{Bu}$ & $\mathrm{N}$ & 63 & {$[92]$} \\
$\mathbf{6 9 0}$ & $4-\mathrm{Cl}-\mathrm{Ph}$ & $\mathrm{t}-\mathrm{Bu}$ & $\mathrm{N}$ & 68 & {$[92]$} \\
$\mathbf{6 9}$ & $\mathrm{Ph}$ & $\mathrm{Me}$ & $\mathrm{Me}$ & 61 & {$[92]$} \\
$\mathbf{6 9}$ & $4-\mathrm{F}-\mathrm{Ph}$ & $\mathrm{Me}$ & $\mathrm{Me}$ & 59 & {$[92]$} \\
$\mathbf{6 9}$ & $\mathrm{Ph}$ & $\mathrm{Mes}$ & $\mathrm{H}$ & 66 & {$[92]$} \\
$\mathbf{6 9 s}$ & $4-\mathrm{Cl}-\mathrm{Ph}$ & $\mathrm{Me}$ & $\mathrm{H}$ & 72 & {$[92]$} \\
\hline
\end{tabular}

Separate catalyzed reaction of imidazopyrimidines $\mathbf{7 0}$ with diaryl acetylenes gave library of compounds with anti-inflammatory activity [11], Scheme 35, Table 17.
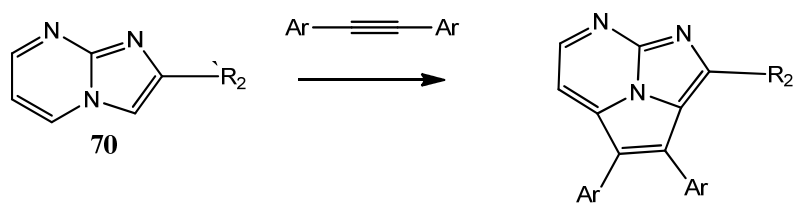

Scheme 35. DMF, PEG-1500, sealed tube $\mathrm{Pd}(\mathrm{OAc})_{2}, \mathrm{Cu}(\mathrm{OAc})_{2}, \mathrm{TBAB} 90^{\circ} \mathrm{C}, 12 \mathrm{~h}$.

Table 17. Substituents and yields of reaction of imidazopyrimidines and diarylacetylenes (Scheme 35).

\begin{tabular}{ccccc}
\hline $\mathbf{N}$ & $\mathbf{A r}$ & $\mathbf{R} 2$ & Yield, $\%$ & Ref. \\
\hline $\mathbf{7 0 a}$ & $\mathrm{Ph}$ & $\mathrm{Ph}$ & 76 & {$[11]$} \\
$\mathbf{7 0 b}$ & $\mathrm{Ph}$ & 2-MeOPh & 72 & {$[11]$} \\
$\mathbf{7 0 c}$ & $4-\mathrm{FPh}$ & 2-MeOPh & 78 & {$[11]$} \\
$\mathbf{7 0 d}$ & $\mathrm{E}$ & $2-\mathrm{MeOPh}$ & 74 & {$[11]$} \\
$\mathbf{7 0 e}$ & $\mathrm{E}$ & $4-\mathrm{MeOPh}$ & 70 & {$[11]$} \\
$\mathbf{7 0 f}$ & $4-\mathrm{FPh}$ & $3-\mathrm{CNPh}$ & 61 & {$[11]$} \\
$\mathbf{7 0 g}$ & $\mathrm{Ph}$ & $3-\mathrm{CNPh}$ & 82 & {$[11]$} \\
$\mathbf{7 0 h}$ & $2-\mathrm{Pyridyl}$ & $3-\mathrm{CNPh}$ & 65 & {$[11]$} \\
$\mathbf{7 0 i}$ & $4-\mathrm{MePh}$ & $4-\mathrm{CNPh}$ & 68 & {$[11]$} \\
$\mathbf{7 0 j}$ & $\mathrm{Ph}$ & $2-\mathrm{NO} 2 \mathrm{Ph}$ & 64 & {$[11]$} \\
$\mathbf{7 0 k}$ & $\mathrm{Ph}$ & $4-\mathrm{MeOPh}$ & 74 & {$[11]$} \\
$\mathbf{7 0 1}$ & $4-\mathrm{MePh}$ & $4-\mathrm{MeOPh}$ & 65 & \\
\hline
\end{tabular}

The new class of excited-state intramolecular proton transfer-capable molecules, benzo[a]cyclazines, bearing the 2-hydroxyphenyl substituent were prepared in a straightforward manner from imidazo[1,2-a]pyridines 71 via a tandem [8+2] cycloaddition-[2+6+2] dehydrogenation reaction using microwave [6], and similar reaction also involved imidazopyrimidine derivatives 72 [93], Scheme 36, Table 18. 


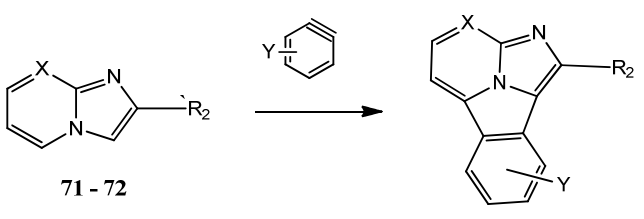

Scheme 36. Reaction of azaindolizines with benzynes (Table 18).

Table 18. Substituents and yields of reaction of azaindolizines and benzynes (Scheme 36).

\begin{tabular}{|c|c|c|c|c|c|}
\hline $\mathbf{N}$ & R2 & $x$ & $Y^{*}$ & Yield, \% & Ref. \\
\hline $71 a$ & 2-HOPh & $\mathrm{H}$ & A & 23 & [6] \\
\hline $71 b$ & 2-HO-4-MeOPh & $\mathrm{H}$ & A & 21 & [6] \\
\hline $71 c$ & 2-HO-5-FPh & $\mathrm{H}$ & A & 19 & [6] \\
\hline $71 d$ & 2-HO-5-MePh & $\mathrm{H}$ & A & 21 & [6] \\
\hline $72 a$ & $\mathrm{Ph}$ & $\mathrm{H}$ & B & 74 & [93] \\
\hline $72 b$ & 4-MeOPh & $\mathrm{H}$ & B & 39 & [93] \\
\hline $72 c$ & 4-FPh & $\mathrm{H}$ & B & 49 & [93] \\
\hline $72 d$ & 4-NO2Ph & $\mathrm{H}$ & B & 51 & [93] \\
\hline $72 e$ & $3,5-(\mathrm{BnO}) 2 \mathrm{Ph}$ & $\mathrm{H}$ & B & 49 & [93] \\
\hline $72 \mathrm{f}$ & $\mathrm{Ph}$ & $\mathrm{N}$ & B & 58 & [93] \\
\hline $72 \mathrm{~g}$ & 4-MeOPh & $\mathrm{N}$ & B & 54 & [93] \\
\hline $72 \mathrm{~h}$ & 4-FPh & $\mathrm{N}$ & B & 38 & [93] \\
\hline $72 \mathbf{i}$ & 4-MeOPh & $\mathrm{H}$ & $\mathrm{C}$ & $43\left(3^{\prime}\right)$ & [93] \\
\hline $72 \mathrm{j}$ & 4-MeOPh & $\mathrm{N}$ & $\mathrm{C}$ & $34\left(3^{\prime}\right)$ & [93] \\
\hline $72 \mathrm{k}$ & 4-MeOPh & $\mathrm{H}$ & $\mathrm{D}$ & $39\left(4^{\prime}\right)$ & [93] \\
\hline 721 & 4-MeOPh & $\mathrm{H}$ & $\mathrm{D}$ & $18\left(5^{\prime}\right)$ & [93] \\
\hline $72 \mathrm{~m}$ & $E^{\prime}$ & $\mathrm{H}$ & B & 51 & [93] \\
\hline
\end{tabular}

* Not determined.

A-1-TMS-2-OSO ${ }_{2} \mathrm{CF}_{3}$-benzene, $\mathrm{CsF}$, 18-Crown-6, MW (25 min, $160^{\circ} \mathrm{C}$ ); B-1-TMS2- $\mathrm{OSO}_{2} \mathrm{CF}_{3}$-benzene, $\mathrm{CsF}$, 18-Crown-6, $\mathrm{MW}\left(90 \mathrm{~W}, 40 \mathrm{psi}, 15 \mathrm{~min}, 80^{\circ} \mathrm{C}\right.$ ); C-1-TMS-2$\mathrm{OSO}_{2} \mathrm{CF}_{3}$-3-MeO-benzene, CsF, 18-Crown-6, MW ( $90 \mathrm{~W}, 50 \mathrm{psi}, 15 \mathrm{~min}, 80^{\circ} \mathrm{C}$ ); D-1-TMS2- $\mathrm{OSO}_{2} \mathrm{CF}_{3}$-4-MeO-benzene, $\mathrm{CsF}$, 18-Crown-6, $\mathrm{MW}\left(90 \mathrm{~W}, 50 \mathrm{psi}, 15 \mathrm{~min}, 80^{\circ} \mathrm{C}\right.$ )

A base promoted protocol for the synthesis of benzo[a]cyclazines from imidazopyridines and benzyne precursors under metal-free conditions was developed [94], Scheme 37, Table 19.<smiles>[R]c1cn2cc([R])c([2H])c([R])c2n1</smiles>

Scheme 37. Reaction of imidazopyridines with benzynes (Table 19).

Table 19. Substituents and yields of reaction of imidazopiridines and benzynes (Scheme 37).

\begin{tabular}{|c|c|c|c|c|c|c|c|c|}
\hline $\mathbf{N}$ & R2 & R6 & R7 & R8 & Method * & Reagent & Yield, \% & Ref. \\
\hline $73 a$ & $\mathrm{Ph}$ & $\mathrm{H}$ & $\mathrm{H}$ & $\mathrm{H}$ & A & [Benzyne] & 75 & [94] \\
\hline $73 b$ & 4-MeO-Ph & $\mathrm{H}$ & $\mathrm{H}$ & $\mathrm{H}$ & A & [Benzyne] & 72 & {$[94]$} \\
\hline $73 c$ & 4-Et-Ph & $\mathrm{H}$ & $\mathrm{H}$ & $\mathrm{H}$ & A & [Benzyne] & 81 & [94] \\
\hline $73 d$ & 4-F-Ph & $\mathrm{H}$ & $\mathrm{H}$ & $\mathrm{H}$ & A & [Benzyne] & 69 & [94] \\
\hline $73 e$ & 4-CN-Ph & $\mathrm{H}$ & $\mathrm{H}$ & $\mathrm{H}$ & A & [Benzyne] & 82 & [94] \\
\hline $73 \mathrm{f}$ & 2-F-Ph & $\mathrm{H}$ & $\mathrm{H}$ & $\mathrm{H}$ & A & [Benzyne] & 70 & [94] \\
\hline $73 \mathrm{~g}$ & 2-Cl-Ph & $\mathrm{H}$ & $\mathrm{H}$ & $\mathrm{H}$ & A & [Benzyne] & 66 & [94] \\
\hline $73 \mathrm{~h}$ & 2-Me-Ph & $\mathrm{H}$ & $\mathrm{H}$ & $\mathrm{H}$ & A & [Benzyne] & 71 & [94] \\
\hline $73 \mathbf{i}$ & $2-\mathrm{Br}-\mathrm{Ph}$ * & $\mathrm{H}$ & $\mathrm{H}$ & $\mathrm{H}$ & A & [Benzyne] & 55 (de-brom) & {$[94]$} \\
\hline $73 j$ & $4-\mathrm{Cl}-\mathrm{Ph}$ & $\mathrm{Me}$ & $\mathrm{H}$ & $\mathrm{H}$ & A & [Benzyne] & 83 & [94] \\
\hline
\end{tabular}


Table 19. Cont.

\begin{tabular}{|c|c|c|c|c|c|c|c|c|}
\hline $\mathbf{N}$ & R2 & R6 & R7 & R8 & Method * & Reagent & Yield, \% & Ref. \\
\hline $73 \mathrm{k}$ & 2-Cl-Ph & $\mathrm{H}$ & $\mathrm{H}$ & $\mathrm{Me}$ & A & [Benzyne] & 63 & [94] \\
\hline 731 & $\mathrm{Ph}$ & $\mathrm{Me}$ & $\mathrm{H}$ & $\mathrm{H}$ & A & [Benzyne] & 71 & [94] \\
\hline $73 \mathrm{~m}$ & 4-Et-Ph & $\mathrm{H}$ & $\mathrm{H}$ & $\mathrm{Me}$ & A & [Benzyne] & 65 & [94] \\
\hline $73 n$ & 4-Et-Ph & $\mathrm{Me}$ & $\mathrm{H}$ & $\mathrm{H}$ & A & [Benzyne] & 60 & [94] \\
\hline 730 & 4-MeO-Ph & $\mathrm{Me}$ & $\mathrm{H}$ & $\mathrm{H}$ & A & [Benzyne] & 72 & [94] \\
\hline $73 p$ & 2-F-Ph & $\mathrm{H}$ & $\mathrm{H}$ & $\mathrm{Me}$ & A & [Benzyne] & 60 & [94] \\
\hline $73 q$ & $\mathrm{Ph}$ & $\mathrm{H}$ & $\mathrm{H}$ & $\mathrm{Me}$ & A & [Benzyne] & 75 & [94] \\
\hline $73 r$ & 4-Cl-Ph & $\mathrm{H}$ & $\mathrm{Me}$ & $\mathrm{H}$ & A & [Benzyne] & 32 & [94] \\
\hline $73 \mathrm{~s}$ & 4 -Et-Ph & $\mathrm{Br} *$ & $\mathrm{H}$ & $\mathrm{H}$ & A & [Benzyne] & 54 (de-brom) & [94] \\
\hline $73 t$ & $\mathrm{H}$ & $\mathrm{H}$ & $\mathrm{CO} 2 \mathrm{Me}$ & $\mathrm{H}$ & A & [Benzyne] & 50 & [94] \\
\hline $73 u$ & $\mathrm{H}$ & $\mathrm{Cl}$ & $\mathrm{H}$ & $\mathrm{H}$ & A & [Benzyne] & 78 & [94] \\
\hline $73 v$ & $\mathrm{H}$ & $\mathrm{H}$ & $\mathrm{H}$ & $\mathrm{H}$ & A & [Benzyne] & 45 & [94] \\
\hline $73 x$ & $\mathrm{H}$ & $\mathrm{H}$ & $\mathrm{H}$ & $\mathrm{H}$ & B & [1-MeO-benzyne-2] & 70 & [94] \\
\hline $73 y$ & $\mathrm{H}$ & $\mathrm{H}$ & $\mathrm{H}$ & $\mathrm{H}$ & $\mathrm{C}$ & [1-Me-benzyne-3] & 65 (2 isom.) & [94] \\
\hline 73 & 4-Et-Ph & $\mathrm{H}$ & $\mathrm{H}$ & $\mathrm{H}$ & $\mathrm{C}$ & [1-Me-benzyne-3] & 72 (2 isom.) & [94] \\
\hline $73 z$ & 4-Cl-Ph & $\mathrm{H}$ & $\mathrm{H}$ & $\mathrm{H}$ & $\mathrm{C}$ & [1-Me-benzyne-3] & 69 (2 isom.) & [94] \\
\hline 73a1 & 4-CN-Ph & $\mathrm{H}$ & $\mathrm{H}$ & $\mathrm{H}$ & $\mathrm{D}$ & [1-MeO-benzyne-2] & $80\left(3^{\prime}\right)$ & [94] \\
\hline $73 \mathrm{~b} 1$ & 4-CN-Ph & $\mathrm{H}$ & $\mathrm{H}$ & $\mathrm{H}$ & E & [1-Me-benzyne-3] & 75 (2 isom.) & [94] \\
\hline $73 \mathrm{c} 1$ & 4-Et-Ph & $\mathrm{H}$ & $\mathrm{H}$ & $\mathrm{H}$ & $\mathrm{F}$ & [1-MeO-benzyne-2] & 73 & [94] \\
\hline $73 \mathrm{~d} 1$ & $\mathrm{t}-\mathrm{Bu}$ & $\mathrm{H}$ & $\mathrm{H}$ & $\mathrm{H}$ & G & [Benzyne] & 62 & [94] \\
\hline
\end{tabular}

${ }^{*} \mathrm{~A}$-1-TMS-2-OSO $\mathrm{CF}_{3}$-benzene 18-crown-6-ether, $\mathrm{K}_{2} \mathrm{CO}_{3}$; acetone, $45^{\circ} \mathrm{C}, 24 \mathrm{~h}$; B-1-TMS-2-OSO $\mathrm{CF}_{3}-3-\mathrm{MeO}$ benzene; C-1-TMS-2-OSO $\mathrm{CF}_{3}$-4-Me-benzene; D-1-TMS-2-OSO $\mathrm{CF}_{3}$-3-MeO-benzene; E-1-TMS-2-OSO $\mathrm{CF}_{3}$-4Me-benzene; $\mathrm{F}-1$-TMS-2- $\mathrm{OSO}_{2} \mathrm{CF}_{3}$-3-MeO-benzene; G-1-TMS-2- $\mathrm{OSO}_{2} \mathrm{CF}_{3}$-benzene.

An interesting reaction that formally fit the [8+2] cycloaddition was developed for interaction of imidazopyridines 74 and 1,2-dihalobenzenes in presence of Pd-catalyst [95], Scheme 38. Table 20.<smiles>[R]c1cn2cc([R])c([R])c([R])c2n1</smiles><smiles>[X]c1ccccc1[X]</smiles><smiles>[R]C1=C([R])c2nc([R])c3c(c4c([Y])cccc4n23)=C1[R]</smiles>

Scheme 38. Pd/xphos, $\mathrm{K}_{2} \mathrm{CO}_{3}, \mathrm{DMF}, 160{ }^{\circ} \mathrm{C}, 24 \mathrm{~h}$.

Table 20. Substituents and yields of reaction of imidazopyridines and 1,2-dihalobenzenes (Scheme 38).

\begin{tabular}{|c|c|c|c|c|c|c|c|}
\hline $\mathbf{N}$ & $\mathbf{R} 2$ & R6 & R7 & R8 & Yield, \% & Benzene * & Ref \\
\hline $74 a$ & $\mathrm{Ph}$ & $\mathrm{H}$ & $\mathrm{H}$ & $\mathrm{H}$ & 77 & $\mathrm{~A}$ & [95] \\
\hline $74 b$ & 4-Me-Ph & $\mathrm{H}$ & $\mathrm{H}$ & $\mathrm{H}$ & 79 & A & [95] \\
\hline $74 c$ & 4-MeOPh & $\mathrm{H}$ & $\mathrm{H}$ & $\mathrm{H}$ & 54 & A & [95] \\
\hline $74 d$ & 4-ClPh & $\mathrm{H}$ & $\mathrm{H}$ & $\mathrm{H}$ & 45 & A & [95] \\
\hline $74 \mathrm{e}$ & 4-CF3Ph & $\mathrm{H}$ & $\mathrm{H}$ & $\mathrm{H}$ & 74 & A & [95] \\
\hline $74 \mathrm{f}$ & 3-MeOPh & $\mathrm{H}$ & $\mathrm{H}$ & $\mathrm{H}$ & 83 & A & [95] \\
\hline $74 \mathrm{~g}$ & 2-MePh & $\mathrm{H}$ & $\mathrm{H}$ & $\mathrm{H}$ & 60 & A & [95] \\
\hline $74 \mathrm{~h}$ & 2-Naphtyl & $\mathrm{H}$ & $\mathrm{H}$ & $\mathrm{H}$ & 52 & A & [95] \\
\hline $74 \mathbf{i}$ & $\mathrm{Ph}$ & $\mathrm{H}$ & $\mathrm{H}$ & $\mathrm{Me}$ & 83 & A & [95] \\
\hline $74 \mathbf{j}$ & $\mathrm{Ph}$ & $\mathrm{H}$ & $\mathrm{Me}$ & $\mathrm{H}$ & 87 & A & [95] \\
\hline $74 \mathrm{k}$ & $\mathrm{Ph}$ & $\mathrm{H}$ & $\mathrm{Cl}$ & $\mathrm{H}$ & 27 & A & [95] \\
\hline 741 & $\mathrm{Ph}$ & $\mathrm{Me}$ & $\mathrm{H}$ & $\mathrm{H}$ & 73 & A & [95] \\
\hline $74 m$ & $\mathrm{Ph}$ & $\mathrm{H}$ & $\mathrm{H}$ & $\mathrm{N}$ & 95 & A & [95] \\
\hline $74 n$ & $\mathrm{Ph}$ & $\mathrm{H}$ & $\mathrm{H}$ & $\mathrm{H}$ & 80 & B & [95] \\
\hline 740 & $\mathrm{Ph}$ & $\mathrm{H}$ & $\mathrm{H}$ & $\mathrm{H}$ & 69 & C & [95] \\
\hline $74 p$ & $\mathrm{Ph}$ & $\mathrm{H}$ & $\mathrm{H}$ & $\mathrm{H}$ & 82 & $\mathrm{D}$ & [95] \\
\hline $74 q$ & $\mathrm{Ph}$ & $\mathrm{H}$ & $\mathrm{H}$ & $\mathrm{H}$ & 86 & $\mathrm{E}$ & [95] \\
\hline
\end{tabular}


The system containing two fused imidazopyridines 75 was placed in reaction with DMAD [96], Scheme 39. One ring of imidazopyridine entered into [8+2] cycloaddition with the yields $22-30 \%$ on heating in benzene.

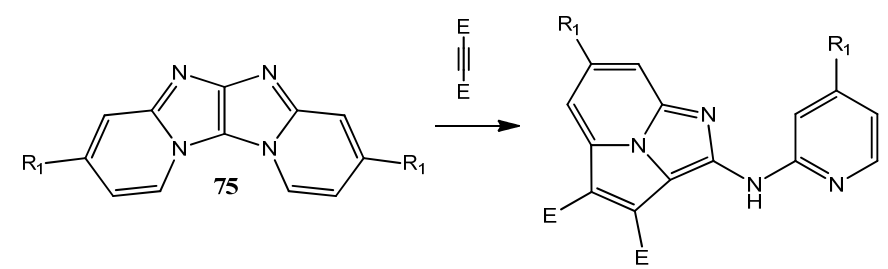

Scheme 39. Cycloaddition of tetraazapentalene derivatives.

A rare example of benzonitrile entered into [8+2] cycloaddition to produce diazacyclazine $\mathbf{7 6 b}$ was reported [97], Scheme 40. Azaindolizine 76a reacted with BuLi giving dipolar structure 76c which underwent cyclization.<smiles>CCc1ncc2ccccn12</smiles>

Scheme 40. Synthesis of diazacyclazine.

\section{Concerted One-Step 1,10 Processes}

If one adds a multiple bond to the end of the tetraene fragment of indolizine, the ring closure becomes possible. A multiple bond can be alkene, alkyne or arene, and the "end" of the tetraene can be position 3 or 5 . However, no such reactions exist for 3-vinyl/ethynyl derivatives and for 5-vinyl indolizines. The first example of such cyclization was reported for 5-ethynyl indolizine 77c [98,99] which is postulated to be intermediate, Scheme 41 .

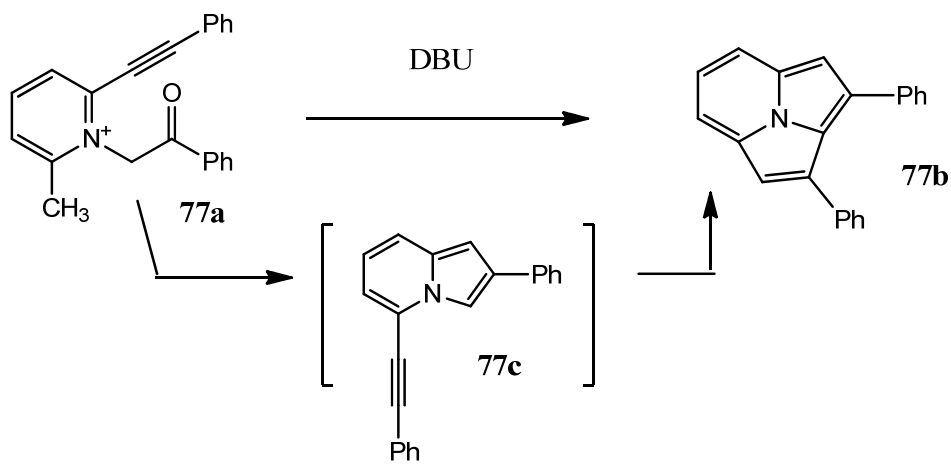

Scheme 41. Cyclization of 6-ethynylpyridinium salts.

According to [98] reaction $\mathbf{7 7 a - 7 7 b}$ proceeded with a yield of $10-15 \%$, later result [99] was $7 \%$. The main product was 5-Me-3-benzoyl indolizine which could not be converted to $77 \mathrm{~b}$. However, we showed that 5-ethynyl indolizine 77c obtained by Sonogashira coupling [100] could not be converted to cyclazine $\mathbf{7 7 b}$ under thermal or acidic conditions.

5-Iodo-indolizine 78a in conditions of Sonogashira reaction with 2 eq of ethoxycarbonyl acetylene gave cyclazine $\mathbf{7 8 b}$ [36], Scheme 42. We supposed that the reaction started from nucleophilic attack of acetylenide anion on $78 \mathrm{c}$. 


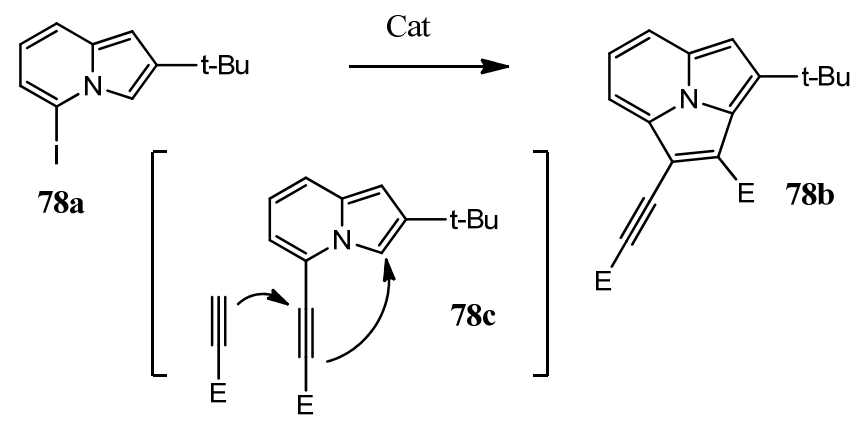

Scheme 42. $\mathrm{Pd}\left(\mathrm{PPh}_{3}\right)_{2} \mathrm{Cl}_{2} / \mathrm{CuI} / \mathrm{MeCN} / \mathrm{Et}_{3} \mathrm{~N} / \mathrm{rt}$.

5-Ethynyl derivatives of imidazopyridines 79a-c behaved in an expected way [99], Scheme 43.

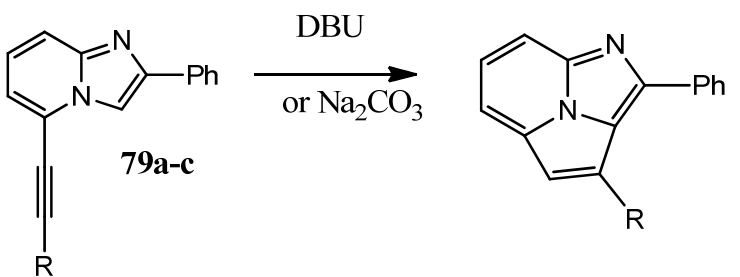

Scheme 43. 79a-c Me, n-Bu, $\mathrm{Ph}(70-80 \%)$.

In one case the double bond of benzene ring at position 3 of indolizine 80a underwent catalytic ring closure to benzocyclazine 80b [35], Scheme 44.<smiles></smiles>

Scheme 44. $\mathrm{Pd}(\mathrm{OAc})_{2} 10 \mathrm{~mol} \% . \mathrm{PPh}_{3}, \mathrm{~K}_{2} \mathrm{CO}_{3} . \mathrm{PhMe} / 115^{\circ} \mathrm{C} / 60 \mathrm{~h}$.

A similar process was employed to obtain highly fluorescent benzo derivatives of azacyclazine starting from Br-substituted 3-aryl imidazopyridines 81, Scheme 45, Table $20[8,101]$.

Table 21. Substituents and yields of the ring closure reaction on Scheme 45.

\begin{tabular}{ccccc}
\hline $\mathbf{N}$ & $\mathbf{A r}$ & $\mathbf{R}$ & Yield, $\%$ & Ref. \\
\hline $\mathbf{8 1 a}$ & $\mathrm{Ph}$ & $\mathrm{H}$ & 100 & {$[8]^{* *},[101]^{*}$} \\
$\mathbf{8 1 \mathbf { b }}$ & $4-(\mathrm{Ph})-\mathrm{Ph}$ & $\mathrm{H}$ & 100 & {$[8]$} \\
$\mathbf{8 1 c}$ & $4-\mathrm{t}-\mathrm{Bu}-\mathrm{Ph}$ & $\mathrm{H}$ & 89 & {$[8]$} \\
$\mathbf{8 1 d}$ & $4-\mathrm{BnO}-\mathrm{Ph}$ & $\mathrm{H}$ & 90 & {$[8]$} \\
$\mathbf{8 1 e}$ & $4-\left(\mathrm{O}\left(\mathrm{CH}_{2}\right)_{2} \mathrm{OBz}\right)-\mathrm{Ph}$ & $\mathrm{H}$ & 89 & {$[8]$} \\
$\mathbf{8 1 f}$ & $4-\mathrm{NMe}-\mathrm{Ph}$ & $\mathrm{H}$ & 98 & {$[8]$} \\
$\mathbf{8 1 g}$ & $4-\mathrm{F}-\mathrm{Ph}$ & $\mathrm{H}$ & 98 & {$[8]$} \\
$\mathbf{8 1 i}$ & $4-\mathrm{Cl}-\mathrm{Ph}$ & $\mathrm{H}$ & 94 & {$[8]$} \\
$\mathbf{8 1 j}$ & $4-\mathrm{CO}_{2} \mathrm{Me}-\mathrm{Ph}$ & $\mathrm{H}$ & 95 & {$[8]$} \\
$\mathbf{8 1 \mathbf { k }}$ & $4-\mathrm{CN}-\mathrm{Ph}$ & $\mathrm{H}$ & 99 & {$[8]$} \\
$\mathbf{8 1 1}$ & $3,4,5-(\mathrm{Me})_{3}-\mathrm{Ph}$ & $\mathrm{H}$ & 93 & {$[8]$} \\
\hline
\end{tabular}


Table 21. Cont.

\begin{tabular}{|c|c|c|c|c|}
\hline $\mathbf{N}$ & Ar & $\mathbf{R 8}$ & Yield, \% & Ref. \\
\hline $81 \mathrm{~m}$ & 2-EtO-4- $\mathrm{NO}_{2}-\mathrm{Ph}$ & $\mathrm{H}$ & 92 & [8] \\
\hline $81 n$ & $2,3,4,5-(\mathrm{F})_{4}-\mathrm{Ph}$ & $\mathrm{H}$ & 66 & [8] \\
\hline 810 & 2-MeO-Ph & $\mathrm{H}$ & 98 & [8] \\
\hline $81 p$ & 1-Naphtyl & $\mathrm{H}$ & 97 & [8] \\
\hline $81 q$ & 2-Fluorenonyl & $\mathrm{H}$ & 61 & [8] \\
\hline $81 r$ & 2-Furyl & $\mathrm{H}$ & 40 & [8] \\
\hline $81 s$ & 2-Tienyl & $\mathrm{H}$ & 65 & [8] \\
\hline $81 t$ & 2-Propenyl & $\mathrm{H}$ & 87 & [8] \\
\hline $81 u$ & 2-Styryl & $\mathrm{H}$ & 28 & [8] \\
\hline
\end{tabular}

${ }^{*} \mathrm{Pd}_{2}(\mathrm{dba})_{3}, \mathrm{HP}(\mathrm{t}-\mathrm{Bu})_{3} \mathrm{BF}_{4}, \mathrm{DMF}, 90{ }^{\circ} \mathrm{C}, 16 \mathrm{~h} .{ }^{* *} \mathrm{Pd}_{2}(\mathrm{dba})_{3}, \mathrm{HP}(\mathrm{t}-\mathrm{Bu})_{3} \mathrm{BF}_{4}, \mathrm{~K}_{2} \mathrm{CO}_{3}, \mathrm{DMF} 120{ }^{\circ} \mathrm{C}, 2.5 \mathrm{~h}$.

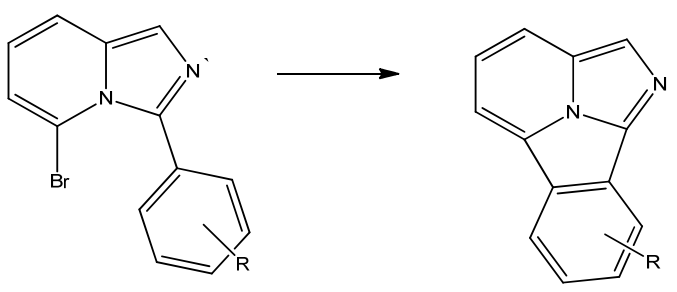

Scheme 45. Cyclization of Br-substituted 3-arylimidazopyridines (Table 21).

We found that 5-chloro-3-benzoyl indolizines $\mathbf{8 2 a , b}$ in acidic conditions closed the ring [102,103], Scheme 46, forming benzocyclazine derivatives 82c, $\mathbf{d}(\mathrm{X}=\mathrm{Cl} 83 \%$, $\mathrm{X}=\mathrm{NO}_{2} 90 \%$ ). Here the protonation opened direct link to 1,10-polyene which underwent ring closure.

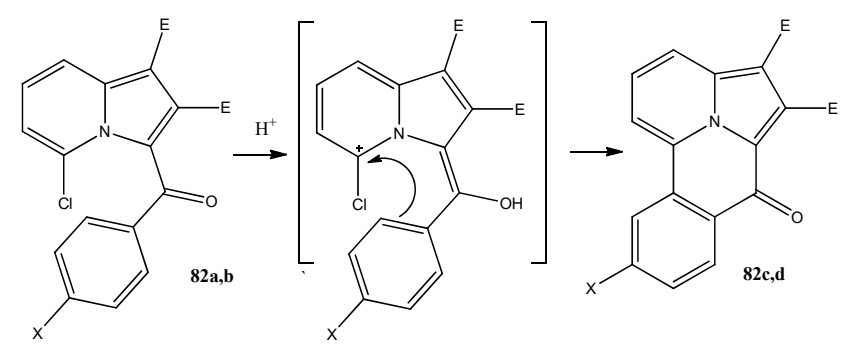

Scheme 46. Unusual ring closure of 5-chlorindolizines.

\section{Concurrence of [8+2] and [4+2] Cycloadditions}

2-Styrylindolizine 83a reacted with methyl acrylate (Scheme 47 ) giving the usual product of oxidative [8+2] cycloaddition-cyclazine $83 \mathrm{~b}$ together with [4+2] cycloadduct $83 \mathrm{c}$ without catalyst [38]. After more prolonged heating (from $122 \mathrm{~h}$ to $288 \mathrm{~h}$ ) the ratio 83b:83c changed from 3:68 to 38:10. A somewhat similar result was obtained in reaction with $\mathrm{N}$-ethylmaleimide where [4+2] adduct $(33 \%)$ was formed together with isomeric dihydrocyclazines $(43 \%)$.<smiles>C=C/C=C/c1cc2ccccn2c1</smiles><smiles>Fc1cc2c(/C=C/c3cc4cccc5n4c3[In]5)cccc2n1-c1ccccc1</smiles>

Scheme 47. Example of concurrence between [8+2] and [8+2] cycloaddition. 
Possibility of concurrence between [8+2] and [4+2] cycloaddition appeared in the case of 2-aryl substituted azaindolizines, Scheme 48. At least three papers appeared on this topic $[7,104,105]$ and the data are summarized in Table 22.
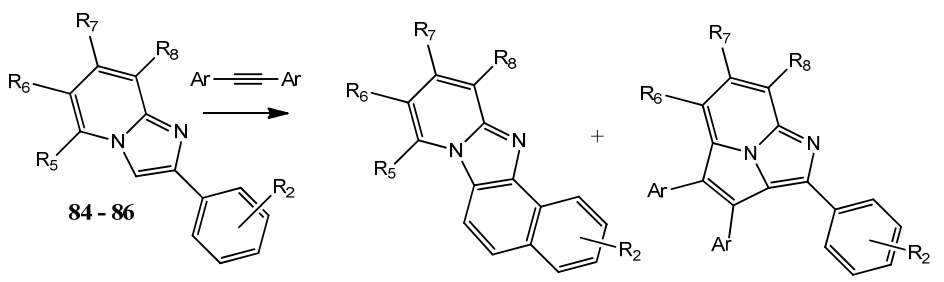

Scheme 48. Concurrence in cycloaddition for 2-arylimidazopyridines (Table 22).

Table 22. Substituents, yields and ratio of isomers in concurrent $2+4$ and $2+8$ cycloadditions of 2-arylazaindolizines and acetylenes (Scheme 48).

\begin{tabular}{|c|c|c|c|c|c|c|c|c|c|}
\hline $\mathbf{N}$ & Ar & Ar & $\mathbf{R} 2$ & R5 & R6 & R7 & R8 & $\begin{array}{c}\text { Yield, \% } \\
(4+2 / 8+2)\end{array}$ & Ref. \\
\hline $84 a$ & $\mathrm{Ph}$ & $\mathrm{Ph}$ & $\mathrm{H}$ & $\mathrm{H}$ & $\mathrm{H}$ & $\mathrm{H}$ & $\mathrm{H}$ & $85(82 / 18)$ & {$[104]^{\mathrm{a}}$} \\
\hline $84 \mathrm{~b}$ & 4-FPh & 4-FPh & $\mathrm{H}$ & $\mathrm{H}$ & $\mathrm{H}$ & $\mathrm{H}$ & $\mathrm{H}$ & $79(68 / 32)$ & [104] \\
\hline $84 c$ & 4-Me-Ph & 4-Me-Ph & $\mathrm{H}$ & $\mathrm{H}$ & $\mathrm{H}$ & $\mathrm{H}$ & $\mathrm{H}$ & $89(60 / 40)$ & [104] \\
\hline $84 d$ & $\mathrm{Ph}$ & $\mathrm{Ph}$ & $\mathrm{H}$ & $\mathrm{H}$ & $\mathrm{H}$ & $\mathrm{H}$ & $\mathrm{Me}$ & $88(46 / 54)$ & [104] \\
\hline $84 e$ & $\mathrm{Ph}$ & $\mathrm{Ph}$ & $\mathrm{H}$ & $\mathrm{Me}$ & $\mathrm{H}$ & $\mathrm{H}$ & $\mathrm{H}$ & $74(100 / 0)$ & [104] \\
\hline $85 a$ & $\mathrm{Ph}$ & $\mathrm{Ph}$ & $\mathrm{H}$ & $\mathrm{H}$ & $\mathrm{H}$ & $\mathrm{H}$ & $\mathrm{H}$ & $65 / 18$ & {$[105]^{b}$} \\
\hline $85 b$ & $\mathrm{Ph}$ & $\mathrm{Ph}$ & 4-Me & $\mathrm{H}$ & $\mathrm{H}$ & $\mathrm{H}$ & $\mathrm{H}$ & $72 / 15$ & [105] \\
\hline $85 c$ & $\mathrm{Ph}$ & $\mathrm{Ph}$ & $4-\mathrm{MeO}$ & $\mathrm{H}$ & $\mathrm{H}$ & $\mathrm{H}$ & $\mathrm{H}$ & $75 / 13$ & [105] \\
\hline $85 d$ & $\mathrm{Ph}$ & $\mathrm{Ph}$ & $4-F$ & $\mathrm{H}$ & $\mathrm{H}$ & $\mathrm{H}$ & $\mathrm{H}$ & $50 / 33$ & [105] \\
\hline $85 e$ & $\mathrm{Ph}$ & $\mathrm{Ph}$ & 4-CF3 & $\mathrm{H}$ & $\mathrm{H}$ & $\mathrm{H}$ & $\mathrm{H}$ & $39 / 42$ & [105] \\
\hline $85 \mathrm{f}$ & $\mathrm{Ph}$ & $\mathrm{Ph}$ & 2-Me & $\mathrm{H}$ & $\mathrm{H}$ & $\mathrm{H}$ & $\mathrm{H}$ & $40 / 45$ & [105] \\
\hline $85 \mathrm{~g}$ & $\mathrm{Ph}$ & $\mathrm{Ph}$ & 2-Tienyl & $\mathrm{H}$ & $\mathrm{H}$ & $\mathrm{H}$ & $\mathrm{H}$ & $80 / 9$ & [105] \\
\hline $85 \mathrm{~h}$ & $\mathrm{Ph}$ & $\mathrm{Ph}$ & 1-Naphtyl & $\mathrm{H}$ & $\mathrm{H}$ & $\mathrm{H}$ & $\mathrm{H}$ & $43 / 35$ & [105] \\
\hline $85 \mathrm{i}$ & $\mathrm{Ph}$ & $\mathrm{Ph}$ & $\stackrel{1}{\mathrm{H}}$ & $\mathrm{H}$ & $\mathrm{H}$ & $\mathrm{Me}$ & $\mathrm{H}$ & $55 / 29$ & [105] \\
\hline $85 j$ & $\mathrm{Ph}$ & $\mathrm{Ph}$ & $\mathrm{H}$ & $\mathrm{H}$ & $\mathrm{H}$ & $\mathrm{H}$ & $\mathrm{Me}$ & $60 / 26$ & [105] \\
\hline $85 \mathrm{k}$ & $\mathrm{Ph}$ & $\mathrm{Ph}$ & $\mathrm{H}$ & $\mathrm{H}$ & $\mathrm{Cl}$ & $\mathrm{H}$ & $\mathrm{H}$ & $69 / 7$ & [105] \\
\hline 851 & $\mathrm{Ph}$ & $\mathrm{Ph}$ & $\mathrm{H}$ & $\mathrm{H}$ & $\mathrm{CF}_{3}$ & $\mathrm{H}$ & $\mathrm{H}$ & $77 / 0$ & [105] \\
\hline $85 \mathrm{~m}$ & 4-MeO-Ph & 4-MeO-Ph & $\mathrm{H}$ & $\mathrm{H}$ & $\mathrm{H}$ & $\mathrm{H}$ & $\mathrm{H}$ & $61 / 16$ & [105] \\
\hline $85 n$ & 4-Cl-Ph & 4-Cl-Ph & $\mathrm{H}$ & $\mathrm{H}$ & $\mathrm{H}$ & $\mathrm{H}$ & $\mathrm{H}$ & $60 / 20$ & [105] \\
\hline 850 & $4-\mathrm{CF}_{3}-\mathrm{Ph}$ & $4-\mathrm{CF}_{3}-\mathrm{Ph}$ & $\mathrm{H}$ & $\mathrm{H}$ & $\mathrm{H}$ & $\mathrm{H}$ & $\mathrm{H}$ & $63 / 15$ & [105] \\
\hline $85 p$ & 2-Me-Ph & 2-Me-Ph & $\mathrm{Ph}$ & $\mathrm{H}$ & $\mathrm{H}$ & $\mathrm{H}$ & $\mathrm{H}$ & $66 / 20$ & [105] \\
\hline $85 q$ & $E^{\prime}$ & $E^{\prime}$ & $\mathrm{H}$ & $\mathrm{H}$ & $\mathrm{H}$ & $\mathrm{H}$ & $\mathrm{H}$ & $51 / 14$ & [105] \\
\hline $85 r$ & $\mathrm{Ph}$ & $\mathrm{Me}$ & $\mathrm{Ph}$ & $\mathrm{H}$ & $\mathrm{H}$ & $\mathrm{H}$ & $\mathrm{H}$ & $56 / 14$ & [105] \\
\hline $85 s$ & $\mathrm{Ph}$ & $n-P r$ & $\mathrm{Ph}$ & $\mathrm{H}$ & $\mathrm{H}$ & $\mathrm{H}$ & $\mathrm{H}$ & $47 / 16$ & [105] \\
\hline $85 t$ & $\mathrm{Ph}$ & $\mathrm{Ph}$ & $\mathrm{Ph}$ & $\mathrm{Me}$ & $\mathrm{H}$ & $\mathrm{H}$ & $\mathrm{H}$ & $60 / 0$ & [105] \\
\hline $85 u$ & $\mathrm{Ph}$ & $\mathrm{Ph}$ & 4-Me & $\mathrm{Me}$ & $\mathrm{H}$ & $\mathrm{H}$ & $\mathrm{H}$ & $65 / 0$ & [105] \\
\hline $85 v$ & $\mathrm{Ph}$ & $\mathrm{Ph}$ & $4-\mathrm{CF}_{3}$ & $\mathrm{Me}$ & $\mathrm{H}$ & $\mathrm{H}$ & $\mathrm{H}$ & $51 / 0$ & [105] \\
\hline $85 w$ & $\mathrm{Ph}$ & $\mathrm{Ph}$ & $4-\mathrm{F}$ & $\mathrm{Me}$ & $\mathrm{H}$ & $\mathrm{H}$ & $\mathrm{H}$ & $56 / 0$ & [105] \\
\hline $85 x$ & $\mathrm{Ph}$ & $\mathrm{Ph}$ & 2-Me & $\mathrm{Me}$ & $\mathrm{H}$ & $\mathrm{H}$ & $\mathrm{H}$ & $52 / 0$ & [105] \\
\hline $85 y$ & $\mathrm{Ph}$ & $\mathrm{Ph}$ & 3-Me & $\mathrm{Me}$ & $\mathrm{H}$ & $\mathrm{H}$ & $\mathrm{H}$ & $68 / 02$ isomers & [105] \\
\hline $85 z$ & $\mathrm{Ph}$ & $\mathrm{Ph}$ & Mes & $\mathrm{H}$ & $\mathrm{H}$ & $\mathrm{H}$ & $\mathrm{H}$ & $0 / 77$ & [105] \\
\hline $85 \mathrm{a} 1$ & $\mathrm{Ph}$ & $\mathrm{Ph}$ & Mes & $\mathrm{H}$ & $\mathrm{H}$ & $\mathrm{H}$ & $\mathrm{Me}$ & $0 / 81$ & [105] \\
\hline $85 \mathrm{~b} 1$ & $\mathrm{Ph}$ & $\mathrm{Ph}$ & Mes & $\mathrm{H}$ & $\mathrm{H}$ & $\mathrm{Me}$ & $\mathrm{H}$ & $0 / 79$ & [105] \\
\hline $85 \mathrm{c} 1$ & $\mathrm{Ph}$ & $\mathrm{Ph}$ & Mes & $\mathrm{H}$ & $\mathrm{Me}$ & $\mathrm{H}$ & $\mathrm{H}$ & $0 / 77$ & [105] \\
\hline $85 \mathrm{~d} 1$ & $\mathrm{Ph}$ & $\mathrm{Ph}$ & Mes & $\mathrm{H}$ & $\mathrm{H}$ & $\mathrm{MeO}$ & $\mathrm{H}$ & $0 / 86$ & [105] \\
\hline $85 \mathrm{e} 1$ & $\mathrm{Ph}$ & $\mathrm{Ph}$ & Mes & $\mathrm{H}$ & $\mathrm{Cl}$ & $\mathrm{H}$ & $\mathrm{H}$ & $0 / 65$ & [105] \\
\hline $86 a$ & 4-BrPh & 4-BrPh & $\mathrm{Ph}$ & $\mathrm{H}$ & $\mathrm{H}$ & $\mathrm{H}$ & $\mathrm{H}$ & $22 / 27$ & {$[7]^{c}$} \\
\hline $86 b$ & 4-BrPh & 4-BrPh & 4-CNPh & $\mathrm{H}$ & $\mathrm{H}$ & $\mathrm{H}$ & $\mathrm{H}$ & $22 / 35$ & {$[7]^{c}$} \\
\hline
\end{tabular}

a: Pd-cat NHC complex $\mathrm{Cu}(\mathrm{OAc})_{2}, \mathrm{TBAB}, \mathrm{DMA}, 90^{\circ} \mathrm{C}, 12 \mathrm{~h}$; ${ }^{\mathrm{b}}: \mathrm{Pd}(\mathrm{OAc})_{2}, \mathrm{Cu}(\mathrm{OAc})_{2}, \mathrm{O}_{2}, \mathrm{TBAB}, \mathrm{DMF}, 100{ }^{\circ} \mathrm{C}$; $\mathrm{Pd}(\mathrm{OAc})_{2}, \mathrm{Cu}(\mathrm{OAC})_{2}, \mathrm{TBAB}, \mathrm{DMAC}, 90^{\circ} \mathrm{C}$.

\section{Understanding the Mechanism: Michael Adducts, Hydrogenated Structures and Others}

Reactions of [8+2] type of indolizines and their aza/benzo derivatives with acetylenes and alkenes are regioselective due to pronounced polarization of indolizine and (if any) of a multiple bond. Thus, the positive end of the double/triple bond (e.g., in E-C $\equiv \mathrm{CH}$ 
or in $\mathrm{ECH}=\mathrm{CH}_{2}$ ) would be definitely attached to $\pi$-excessive pyrrole carbon $\mathrm{C}-3$ without any exception, as is evident from all the tables. If the alkene/acetylene bears an electrondonating group and indolizine is appropriately polarized (e.g., by additional $6(8)-\mathrm{NO}_{2}$ group), then regioselectivity is again preserved, and electronegative end of the multiple bond would be attached to $\pi$-deficient pyridine carbon $C-5$.

\subsection{Theory}

There are theoretical quantum chemical calculations on [8+2] cycloaddition of alkenes to indolizines $[106,107]$ with a variation of the polar nature of substituents in alkenes and comparing indolizine and 6-nitroindolizine. An ab initio and semiempirical (AM1 and SINDO1) calculations clearly confirm the possibility of three different mechanisms (Scheme 49). The concerted one-step mechanism (iii) is preferable, if there are no polar groups in a dienophile and indolizine. Another type of stepwise cycloaddition (electrophilic addition (i) - nucleophilic ring closure (ii)) should be realized for the case of nitroethylene. The last type of dipolar cycloaddition (nucleophilic addition (iv)—electrophilic ring closure (v)) would be expected for the reaction of 6-nitroindolizine with aminoethylene, Table 23.

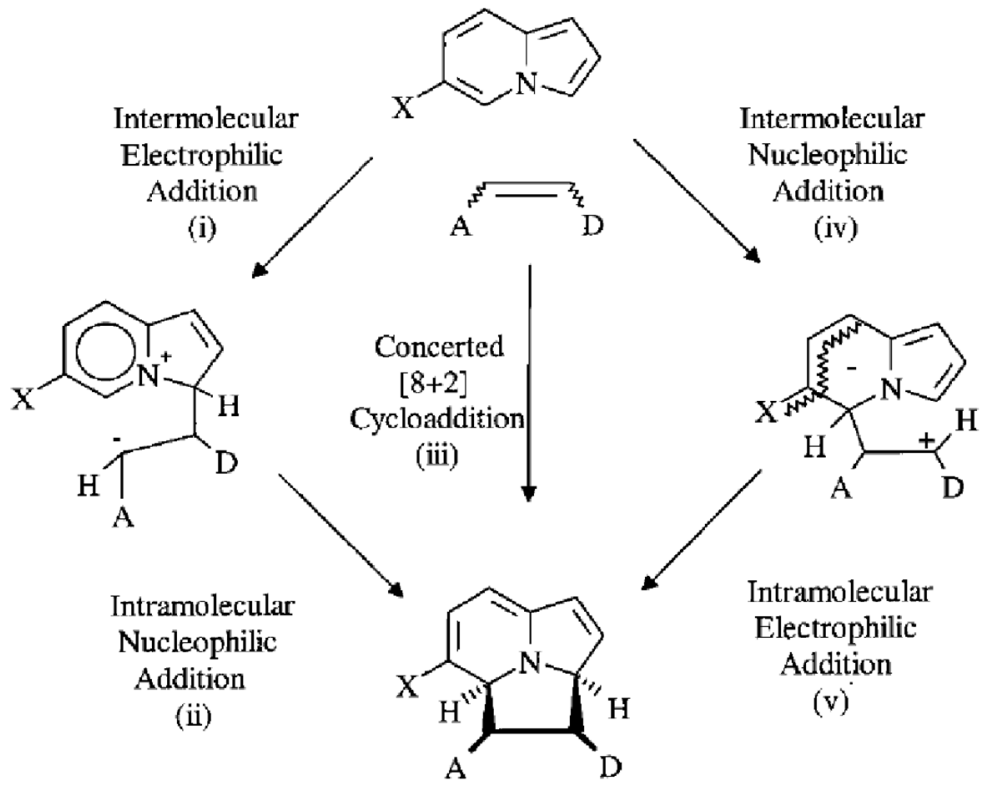

Scheme 49. Theoretically possible mechanisms of [8+2] cycloaddition to indolizines.

Table 23. Possible mechanisms of [8+2] cycloaddition depending on the nature of groups in alkene and indolizne as shown on Scheme 49.

\begin{tabular}{ccc}
\hline Substituent in Alkene & Indolizine & 6-Nitroindolizine \\
\hline Nitroethylene & (i), (ii) & (i), (ii) \\
Methyl acrylate & (ii) & (iii) \\
Acrylonitrile & (iii) & (iii) \\
Ethylene & (iii) & (iv), (v) \\
N,N-Dimethylaminoethylene & (iii) & \\
\hline
\end{tabular}

However, indolizines (even activated by 6- or 8- $\mathrm{NO}_{2}$-group) failed to react with enamines or enols [107], although reaction with dialkylaminoacetylene is possible, Scheme 50. Although the 1:1 adduct was definitely not the product of [8+2] cycloaddition $87 \mathbf{a}$, rather it was [4+2] adduct of acetylene across the nitroethylene $87 \mathrm{~b}$, its structure confirmed the regioselectivity of attack of aminnoacetylene to the position C-5 of indolizine. 


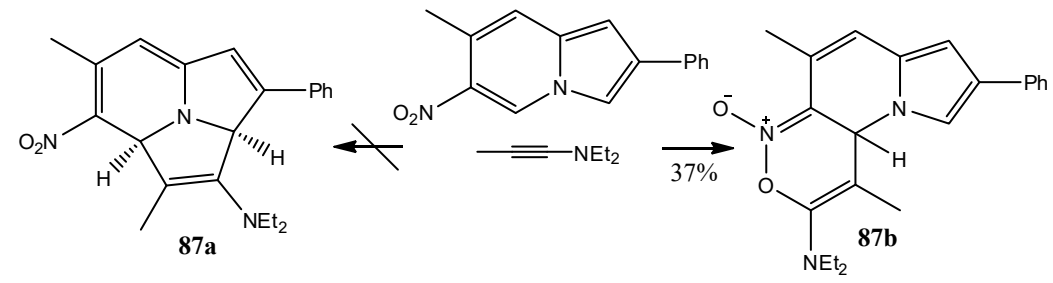

Scheme 50. Abnormal cycloadditon to 6-nitroindolizine.

After the addition of alkyne to position C-3 of indolizine, the initially formed zwitterion $\mathbf{8 8} \mathrm{a}$ could be transformed to a covalent structure either forming the cycloadduct $\mathbf{8 8 b}$ (i.e., dihydrocyclazine) or underwent shift of $\mathrm{H}-3$ from acidic position C-3 to vinyl anion thus forming 3-vynyl derivative 88c. Scheme 51.<smiles>[R]C#C[Y9]([H])([H])C1=CC(/C([R])=C/[R])[n+]2ccccc21</smiles>

Scheme 51. Possible channels of transformation of initially formed zwitter-ion.

\subsection{3-Vinyl Derivatives}

In few cases, 3-vinyl substituted intermediates were isolated and characterized from reactions of indolizines and acetylenes, Scheme 52, Table 24. In the first experiment of reaction of indolizines with DMAD without any catalyst, the cis- and trans-adducts 89 were formed [108]. Cis- and trans-derivatives of pyrrolopyrimidone 90 and DMAD did not undergo further cyclization to azacyclazine in presence of Pd-C [85]. 1.8-Annelatyed indolizines gave purple 3-vinyl adducts 91 with DBZA [46] which underwent further dehydrogenation without cyclization (see Scheme 9). Benzoindolizines 92 [29] and their azaderivative 93 [88] even in presence of catalysis gave the adducts together with cyclazines. 2-Isopropenyl indolizine $\mathbf{9 4}$ after prolonged heating with DMAD gave the mixture of isomeric 3-vinyl derivatives [109]. 

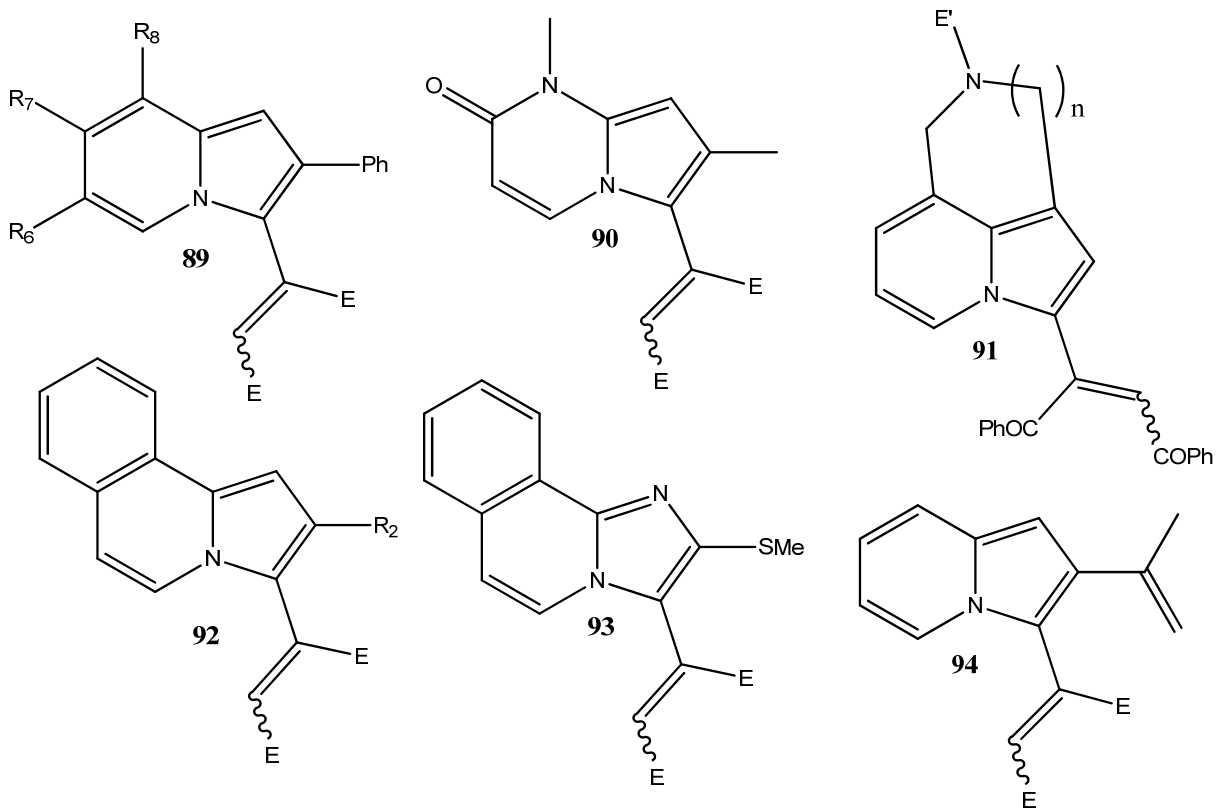

Scheme 52. 3-Vinyl derivatives of aza/benzo/indolizines isolated as intermediates (see Table 24).

Table 24. Stereochemistry, conditions and yields of synthesis of vinyl indolisines on Scheme 52.

\begin{tabular}{ccccc}
\hline $\mathbf{N}$ & $\mathbf{R}$ & Yield (trans/cis), $\%$ & Conditions & Ref. \\
\hline $\mathbf{8 9 a}$ & $\mathrm{R}=\mathrm{H}$ & $13: 4$ & $\mathrm{No} / \mathrm{Me}_{2} \mathrm{CO} / \mathrm{rt} / 15 \mathrm{~h}$ & {$[108]$} \\
$\mathbf{8 9 b}$ & $\mathrm{R} 6=\mathrm{Me}$ & $11: 5$ & & {$[108]$} \\
$\mathbf{8 9 c}$ & $\mathrm{R} 7=\mathrm{Me}$ & $16: 0$ & & {$[108]$} \\
$\mathbf{8 9 d}$ & $\mathrm{R} 8=\mathrm{Me}$ & $20: 0$ & $\mathrm{Pd} / \mathrm{PhMe} / \Delta / 21 \mathrm{~h}$ & {$[108]$} \\
$\mathbf{9 0}$ & & $18: 13$ & $\mathrm{No} / 0^{\circ} / \mathrm{THF}$ & {$[46]$} \\
$\mathbf{9 1 a}$ & $\mathrm{n}=1$ & $62^{*}$ & $\mathrm{No} / 0^{\circ} / \mathrm{THF}$ & {$[46]$} \\
$\mathbf{9 1 b}$ & $\mathrm{n}=2$ & $62^{*}$ & $\mathrm{Pd}-\mathrm{C} / \mathrm{PhMe} / \Delta / 30 \mathrm{~h}$ & {$[29]$} \\
$\mathbf{9 2 a}$ & $\mathrm{R} 2=\mathrm{SMe}$ & $26: 17$ & $\mathrm{Pd}-\mathrm{C} / \mathrm{PhMe} / \Delta / 30 \mathrm{~h}$ & {$[29]$} \\
$\mathbf{9 2 b}$ & $\mathrm{R} 2=\mathrm{H}$ & $0: 11$ & $\mathrm{Pd}-\mathrm{C} / \mathrm{PhMe} / \Delta / 30 \mathrm{~h}$ & {$[88]$} \\
$\mathbf{9 3}$ & & $4^{*}$ & $\mathrm{No} / \mathrm{PhMe} / \mathrm{rt} / 120 \mathrm{~h}$ & {$[109]$} \\
$\mathbf{9 4}$ & & $14^{* *}$ & &
\end{tabular}

${ }^{*}$ Not determined, ${ }^{* *}$ In a mixture.

\subsection{Dihydrocyclazines}

First, dihydrocyclazine was obtained by Boekelheide [23] with a yield of $15 \%$ together with cyclazine. He tried to prove the position of protons by chemical tools and finally assigned the protons to be located as in 95 (Scheme 53), i.e., far from the attached DMAD. In 1984 Japanese chemists tried to prove the structure of all intermediated in the reaction of indolizines with DMAD in the absence of catalyst [108]. They proved two types of structures $96 \mathbf{a}$ and $96 \mathbf{b}$ (together with 3-vinyl adducts 89 ) obtained with the yields $4-27 \%$ for $96 \mathbf{a}$ and $5-6 \%$ for $96 \mathbf{b}$. Bis-(indolizinyl)etane formed the bis-dihydrocyclazine derivative 97 with a yield of $26 \%$ [39]. Azaindolizinone reacted with DMAD in presence of Pd-C giving about $4 \%$ of dihydro-compound 98 [85]. 


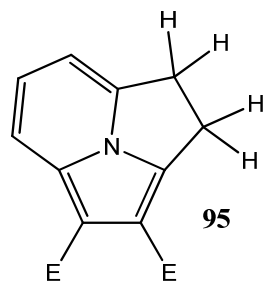<smiles>[R]c1c2n3c(cc(-c4ccccc4)c3[Y]([H])([2H])c1[R])C(F)(F)[C@H]2[R]</smiles>

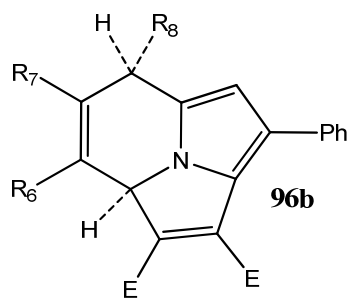<smiles>[CH2]C12CC=Cc3c(F)cc(n31)C(C)=C2CCC1=C(C)C2=CC=C[C@H]1C2</smiles><smiles>CC1=c2c(F)c(F)c3n2C(C1)N(C)C(=O)C=3</smiles>

Scheme 53. Dihydrocyclazines obtained in the synthesis.

The structure of dihydrocyclazine depends on the nature of substituents in the ring. Thus, in our early work [110] we found that 6-nitroindolizine reacted with DMAD $(\mathrm{PhMe} / \Delta / 3 \mathrm{~h}$ ) giving the expected nitrocyclazine 99a (Scheme 54) together with the cyclazine $\mathbf{9 9} \mathbf{b}$ without $\mathrm{NO}_{2}$ group (31\%:7\%), which is formed presumably by elimination of $\mathrm{HNO}_{2}$ from dihydrocyclazine $99 \mathrm{c}$.<smiles></smiles>

Scheme 54. Unusual cycloaddion to 6-nitroindolizine with the loss of $\mathrm{NO}_{2}$ group.

In the paper [111] it was shown that 5-Me-indolizine derivative under the action of DMAD (PhH, rt) gave dihydrocyclazine 100a with a yield of 54\%, Scheme 55. Further reaction with the excess of DMAD give the macrocyclic cyclazine derivative 100b [111] with the structure proved by X-ray, and it was not the structure of the 1:2 adduct (100c) postulated in [108]. 

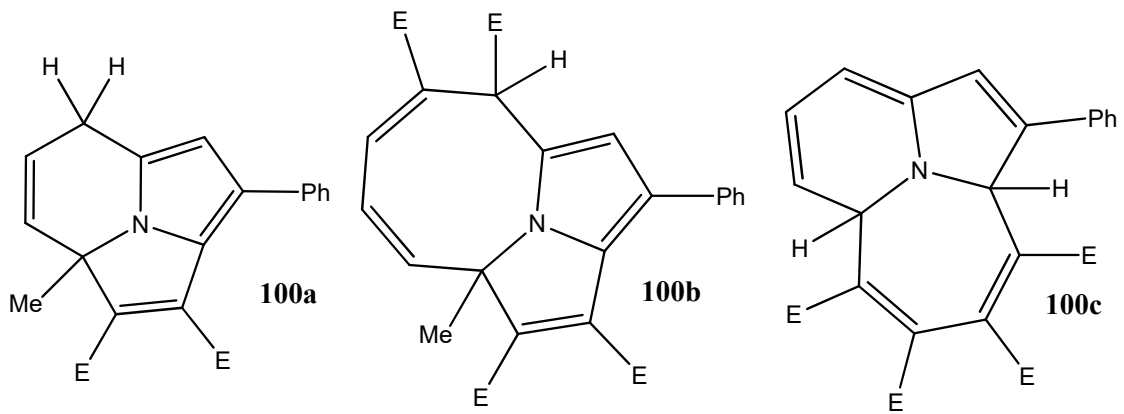

Scheme 55. 1:1 and 1:2 cycloadducts of 5-methylindolizine and DMAD.

The reaction of $\mathrm{Mes}_{2} \mathrm{~B}$-substituted acetylene with benzoindolizine at rt gave dihydrocyclazine 101 with $90 \%$ yield [37] (Scheme 56) which can be further aromatized. The same structure underwent cycloaddition with hetearyl acetylenes [66] $\left(\mathrm{CH}_{2} \mathrm{ClCH}_{2} \mathrm{Cl} / \Delta / 6 \mathrm{~h}\right)$ giving another type of dihydrocyclazines $102(\mathrm{R}=2$-pyridyl, $54 \%$ and $\mathrm{R}=2$-quinoline, $42 \%)$ which were converted to benzocyclazines under the action of sulfur $(\mathrm{PhCl} / \Delta / 10 \mathrm{~h}$ with the yields $59 \%$ and $42 \%$ ). 3-CN substituted benzo[a]indolizine with DMAD (Pd$\mathrm{C} / \mathrm{PhMe} / \Delta / 2 \mathrm{~h}$ ) gave $7 \%$ of the adduct of the structure $\mathbf{1 0 3}$ (together with benzocyclazine) and with di-t-BuOCO-acetylene the yield of cycloadduct is higher (42\%) [65].
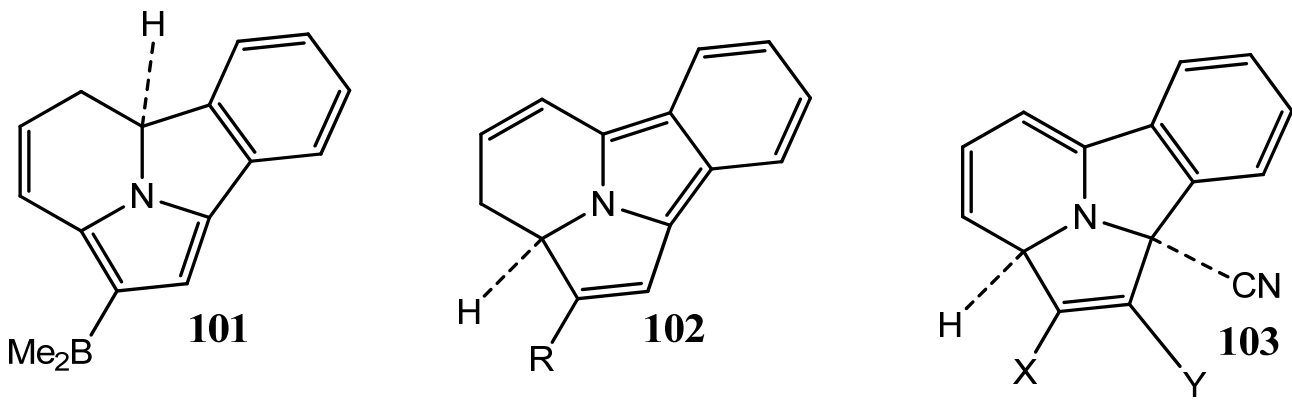

Scheme 56. Different cycloadducts obtained from benzoindolizine.

\subsection{Alkenes}

The reaction of indolizines with alkenes has attracted a lot of attention. The following potential dienophiles were used as $2 \pi$-components for potential [8+2] cycloaddition: nitroolefins, acrylonitrile, benzoquinone, methylvinylketone, alkyl acrylates, alkyl maleate, alkyl fumarate, maleic acid, maleic anhydride, N-substituted maleimide, 4-substituted1,2,4-triazoline-3,5-dione, dialkyl azodicarboxylates, nitrile oxide, 1.2-dicyanocyclobutene and some other [38,53,112-115].

In most reactions, two types of products are observed: first from proton shifts in an intermediate zwitter-ion leading ultimately to the isolated Michael addition product at the position 3 of the indolizine or, second, deriving from hydrogen loss or shifts in the primary adduct giving [2+8] cycloadducts of tetrahydro-, dihydro- or (in rarest cases) aromatic cyclazines.

In particular, indolizines reacted with maleates and acrylates giving [8+2] cycloadducts with the subsequent 1,5-hydrogen shift as in 104a, Scheme 57 [112]. In most other cases Michael adducts at C-3 104b were formed. Benzo[a]indolizines with some dipolarophiles produced kinetically controlled cycloadducts 105a which isomerized to Michael adducts 105b [113]. For further discussion on the mechanism see ref. [116]. 

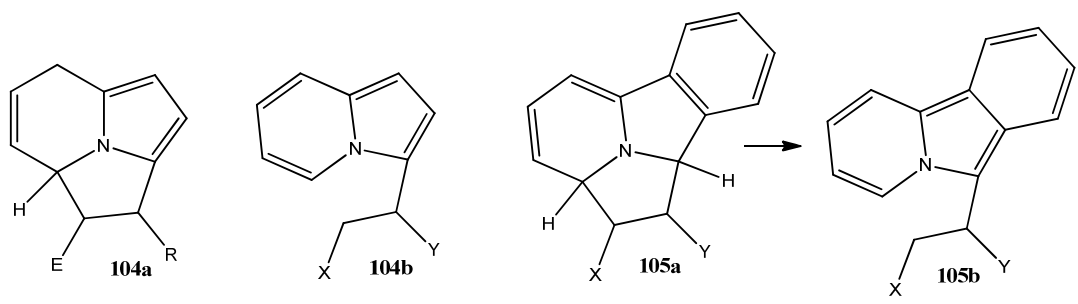

Scheme 57. Structure of some adducts and cycloadducts of indoliznes and alkenes.

\section{Conclusions}

As is evident from all the schemes and tables, [8+2] cycloaddition of indolizines, their aza- and benzo derivatives leading to (aza/benzo) cyclazines is a big portion of modern organic chemistry, its concrete and powerful tool with its own achievements and secrets. There are a lot of catalyst and oxidants proposed to make the final aromatic structure, starting from oxygen, sulfur, $\mathrm{Pd}-\mathrm{C}, \mathrm{Pd}(\mathrm{OAc})_{2}$ and $\mathrm{Pd}$ complexes, $\mathrm{Cu}(\mathrm{OAc})_{2}$, $\mathrm{MnO}_{2}$, quinones (DDQ, benzoquinone), new tools appeared to stimulate reaction (blue LED, microwaves, etc.). The dependence of the process on the nature of substituents in the benzo/aza-substituted indolizines and alkynes/alkenes, the intermediacy of open chains cyclic derivatives made clearer the entire mechanism. Even 60 years after its first discovery, $[8+2]$ cycloadditions continue to play an important part in organic synthesis.

Author Contributions: E.V.B. formulated the goals, managed performance of all work and wrote the review. I.A.S. prepared all tables with the yields. All authors have read and agreed to the published version of the manuscript.

Funding: This research received no external funding.

Conflicts of Interest: The authors declare no conflict of interest.

\section{References}

1. Flitsch, W. Pyrroles with fused six-membered heterocyclic rings: A-fused. In Comprehensive Heterocyclic Chemistry; Katritzky, A., Rees, C.W., Eds.; Pergamon Press: Oxford, UK, 1984; Volume 4, pp. 443-496.

2. Tominaga, Y.; Komiya, K.; Kataoka, S.; Shigemitsu, Y.; Hirota, T.; Sasaki, K. Synthesis of a Luminescent Compound: 8Dimethylaminopyridazino[4,5-a][2.2.3]cyclazine-1,4(2H,3H)-diones. Heterocycles 1988, 48, 1985-1988. [CrossRef]

3. Shen, Y.-M.; Grampp, G.; Leesakul, N.; Hu, H.-W.; Xu, J.-H. Synthesis and Emitting Properties of the Blue-Light Fluorophores Indolizino[3,4,5-ab]isoindole Derivatives. Eur. J. Org. Chem. 2007, 22, 3718-3726. [CrossRef]

4. Granger, D.B. Acenes, Heteroacenes and Analogous Molecules for Organic Photovoltaic and Field Effect Transistor Applications. Ph.D. Thesis, University of Kentucky, Lexington, KY, USA, 2017. [CrossRef]

5. Zeng, C.; Yuan, K.; Wang, N.; Peng, T.; Wu, G.; Wang, S. The opposite and amplifying effect of B $\leftarrow$ N coordination on photophysical properties of regioisomers with an unsymmetrical backbone. Chem. Sci. 2019, 10, 1724-1734. [CrossRef] [PubMed]

6. Stasyuk, A.J.; Banasiewicz, M.; Ventura, B.; Cyrański, M.K.; Gryko, D.T. Benzo[a]imidazo[5,1,2-cd]indolizines-A new class of molecules displaying excited state intramolecular proton transfer. New J. Chem. 2014, 38, 189-197. [CrossRef]

7. Maitra, R.; Chen, J.-H.; Hu, C.-H.; Lee, H.M. Synthesis and Optical Properties of Push-Push-Pull Chromophores Based on Imidazo[5,1,2-cd ]indolizines and Naphtho[1' $\left.2^{\prime}: 4,5\right]$ imidazo[1,2-a]pyridines. Eur. J. Org. Chem. 2017, 40, 5975-5985. [CrossRef]

8. Lévesque, É.; Bechara, W.S.; Constantineau-Forget, L.; Pelletier, G.; Rachel, N.M.; Pelletier, J.N.; Charette, A.B. General C-H Arylation Strategy for the Synthesis of Tunable Visible Light-Emitting Benzo[a]imidazo[2,1,5-c,d]indolizine Fluorophores. J. Org. Chem. 2017, 82, 5046-5067. [CrossRef] [PubMed]

9. Jørgensen, A.S.; Jacobsen, P.; Christiansen, L.B.; Bury, P.S.; Kanstrup, A.; Thorpe, S.M.; Narum, L.; Wassermann, K. Synthesis and estrogen receptor binding affinities of novel pyrrolo[2,1,5-cd]indolizine derivatives. Bioorg. Med. Chem. Lett. 2000, 10, $2383-2386$. [CrossRef]

10. Jørgensen, A.S.; Jacobsen, P.; Christiansen, L.B.; Bury, P.S.; Kanstrup, A.; Thorpe, S.; Bain, S.; Narum, L.; Wassermann, K. Synthesis and pharmacology of a novel pyrrolo[2,1,5-cd]indolizine (NNC 45-0095), a high affinity non-steroidal agonist for the estrogen receptor. Bioorg. Med. Chem. Lett. 2000, 10, 399-402. [CrossRef]

11. Sanivarapu, S.; Vaddiraju, N.; Velide, L. Synthesis and anti-inflammatory activity of 1,2-3-substituted 2a1,4,5-triazacyclopenta[cd] indene derivatives. Med. Chem. Res. 2019, 28, 1461-1470. [CrossRef]

12. Acheson, R.M. Reactions of Acetylenecarboxylic Acids and Their Esters with Nitrogen-Containing Heterocyclic Compounds. Adv. Heterocycl. Chem. 1963, 1, 125-165. [CrossRef]

13. Taurins, A. Special Topics in Heterocyclic Chemistry; Wiley: Chichester, UK, 1977; Volume 30, pp. 245-270. [CrossRef] 
14. Matsumoto, K.; Uchida, T.; Yamaguchi, J. The Chemistry of Cyclazines. J. Syn. Org. Chem. 1977, 35, 739-749. [CrossRef]

15. Flitsch, W.; Kramer, U. Cyclazines and Related N-Bridged Annulenes. Adv. Heterocycl. Chem. 1978, 22, 321-365. [CrossRef]

16. Tominaga, Y.; Shiroshita, Y.; Hosom, A. Synthesis of [2.2.3]Cyclazines, Aza[2.2.3]cyclazines and Their Related Compounds. Heterocycles 1988, 27, 2251-2288. [CrossRef]

17. Beusker, P.H.; Scheeren, H.W. Intermolecular cyclization reactions to form carbocycles. In The Chemistry of Dienes and Polyenes; Rappoport, Z., Ed.; John Wiley \& Sons: Hoboken, NJ, USA, 2000; Volume 2, pp. 329-480.

18. Matsuda, Y. The Chemistry of Cyclazines. J. Pharm. Soc. Jpn. [Yakugaku Zasshi] 2001, 121, 971-988. [CrossRef]

19. Tominaga, Y. Science of Synthesis. Houben-Weyl Methods of Molecular Transformations; Thieme: New York, NY, USA, 2004; Volume 17, pp. 1025-1078. [CrossRef]

20. Nair, V.; Abhilash, K. [8+2] Cycloaddition Reactions in Organic Synthesis. Synlett 2008, 3, 301-312. [CrossRef]

21. Nair, V.; Abhilash, K.G. [8+2] Cycloaddition Reactions in the Construction of Heterocycles. Top. Heterocycl. Chem. 2008, 13, 173-200. [CrossRef]

22. Windgassen, R.J.; Saunders, W.H.; Boekelheide, V. Cyclazines. A New Class of Aromatic Heterocycles. J. Am. Chem. Soc. 1959, 81, 1459-1465. [CrossRef]

23. Galbraith, A.; Small, T.; Barnes, R.A.; Boekelheide, V. The Formation of Cycl[3.2.2]azine Derivatives via the Reaction of Pyrrocoline with Dimethyl Acetylenedicarboxylate. J. Am. Chem. Soc. 1961, 83, 453-458. [CrossRef]

24. Boekelheide, V.; Small, T. A Correlation of Some Electrophilic Substitution Reactions of Cycl[3.2.2]azine1. J. Am. Chem. Soc. 1961, 83, 462-463. [CrossRef]

25. Boekelheide, V.; Fahrenholtz, K. The Formation of Pyrrocolines by the Reaction of Dimethyl Acetylenedicarboxylate with Heterocyclic Zwitterions. J. Am. Chem. Soc. 1961, 83, 458-462. [CrossRef]

26. Starikov, A.S.; Kalashnikov, V.V.; Tarakanov, P.A.; Simakov, A.O.; Simonov, S.V.; Tkachev, V.V.; Yarkov, A.V.; Kazachenko, V.P.; Chernyak, A.V.; Zhurkin, F.E.; et al. Synthesis of 1,2-Dicyano-3-arylcycl[3.2.2]azines-First 1,2-Dicarbonitriles Based on Cyclazine Heterocycle. Eur. J. Org. Chem. 2020, 5852-5856. [CrossRef]

27. Jackman, L.; Porter, Q.; Underwood, G. Studies of aromaticity by nuclear magnetic resonance spectroscopy. IV. The proton chemical shifts in Cycl[2,2,3]azine. Aust. J. Chem. 1965, 18, 1221-1226. [CrossRef]

28. Smith, J.O.; Mandal, B.K. A convenient synthesis of 8-substituted indolizines as precursors to 5-substituted cycl\{3.2.2\}azine derivatives. J. Heterocycl. Chem. 1997, 34, 1441-1446. [CrossRef]

29. Tominaga, Y.; Shiroshita, Y.; Kurokawa, T.; Gotou, H.; Matsuda, Y.; Hosomi, A. Synthesis of cycl[3.2.2]azine and benzo[g]cycl[3.2.2] azine derivatives by use of the [2+8] cycloaddition reaction of indolizines and dimethyl acetylenedicarboxylate. J. Heterocycl. Chem. 1989, 26, 477-487. [CrossRef]

30. Maseda, C.; Sone, M.; Tominaga, Y.; Natsuki, R.; Matsuda, Y. Synthesis of Indolizine Derivatives and Their Reactions. J. Pharm. Soc. Jpn. [Yakugaku Zasshi] 1974, 94, 839-843. [CrossRef]

31. Matsuda, Y.; Gotou, H.; Katou, K.; Matsumoto, H.; Yamashita, M.; Takahashi, K.; Ide, I. A New Synthesis of 2,12Dithia[3.3](1,4)cycl[3.2.2]azinophanes. Heterocycles 1990, 31, 983-986. [CrossRef]

32. Matsuda, Y.; Katou, K.; Matsumoto, H.; Nishiyori, T.; Uemura, T.; Urakami, M. Synthesis of 2,12-Dithia[3](2,6)pyridino[3](1,4)cycl azinophane and It's Barrier to Methylene Twist. Heterocycles 1996, 43, 1633-1640. [CrossRef]

33. Babaev, E.V. Fluorinated Indolizines. In Fluorine in Heterocyclic Chemistry; Nenajdenko, V., Ed.; Springer: Wien, Austria, 2014; Volume 1, pp. 157-180. ISBN 978-3-7091-0689-1.

34. Motornov, V.; Tabolin, A.; Nelyubina, Y.V.; Nenajdenko, V.; Ioffe, S. Copper-mediated oxidative [3+2]-annulation of nitroalkenes and pyridinium ylides: A general access to functionalized indolizines. Efficient synthesis of 1-fluoroindolizines. Org. Biomol. Chem. 2019, 17, 1442-1454. [CrossRef]

35. Lepitre, T.; Le Biannic, R.; Othman, M.; Lawson, A.M.; Daïch, A. Metal-Free Cascade Approach toward Polysubstituted Indolizines from Chromone-Based Michael Acceptors. Org. Lett. 2017, 19, 1978-1981. [CrossRef]

36. Babaev, E.V.; Shadrin, I.A.; Rybakov, V.B. (Aza)indolizines and ethyl propiolate: [8+2] and [1,10] cyclizations. Chem. Heterocycl. Comp. 2018, 54, 339-343. [CrossRef]

37. Yang, D.-T.; Radtke, J.; Mellerup, S.K.; Yuan, K.; Wang, X.; Wagner, M.; Wang, S. One-Pot Synthesis of Brightly Fluorescent Mes2B-Functionalized Indolizine Derivatives via Cycloaddition Reactions. Org. Lett. 2015, 17, 2486-2489. [CrossRef] [PubMed]

38. Siberdt, F.; Nasielski, J. Indolizines. 2. The reaction of 2- $\beta$-styrylindolizine with dienophiles. Bull. Soc. Chim. Belg. 1997, 106, 29-38.

39. Goto, H.; Kurata, K.; Awaya, H.; Tominaga, Y.; Matsuda, Y.; Kobayashi, G. Studies on Indolizine Derivatives. VI. Synthesis of Cycl[3.2.2] azinophane Derivative. Heterocycles 1982, 17, 325-329. [CrossRef]

40. Matsuda, Y.; Kohra, S.; Katou, K.; Itou, T.; Uemura, T. Synthesis of a New Annulenoannulene, Cycl[3.2.2]azino[1,2a]cycl[3.2.2]azine. Heterocycles 1997, 45, 2223-2228. [CrossRef]

41. Matsuda, Y.; Kohra, S.; Katou, K.; Itou, T.; Uemura, T. Synthesis of a New Annulenoannulenone, 3H-Cycl[3.2.2]azino[2,1e]cycl[3.3.2]azin-3-one. Heterocycles 1998, 48, 2573-2579. [CrossRef]

42. Noguchi, M.; Tanigawa, N.; Kajigaeshi, S. Preparation of cyclohepta and benzo[ef]cycl[3.2.2]azines. J. Heterocycl. Chem. 1985, 22, 1049-1053. [CrossRef]

43. Noguchi, M.; Tamai, R.; Tanigawa, N.; Okumura, H.; Kajigaeshi, S. Synthesis and properties of cyclohepta[hi]pyrrolo-[2,1,5cd]indolizines. Bull. Chem. Soc. Jpn. 1987, 60, 969-975. [CrossRef] 
44. Noguchi, M.; Yamamoto, T.; Kajigaeshi, S.; Saito, K.; Arai, T. Synthesis and Properties of 4H-Benzo[hi]pyrrolo[2,1,5-cd]indolizin4-ones. Bull. Chem. Soc. Jpn. 1988, 61, 423-429. [CrossRef]

45. Noguchi, M.; Yamamoto, T.; Kajigaeshi, S. Synthesis and Properties of 6H-Benzo[hi]pyrrolo[2,1,5-cd]indolizin-6-ones. Heterocycles 1990, 31, 563-568. [CrossRef]

46. Noguchi, M.; Tanigawa, N.; Kajigaeshi, S. Preparation of Pyrido- and Azepino-fused Cycl[3.2.2]azines. Bull. Chem. Soc. Jpn. 1985, 58, 3444-3447. [CrossRef]

47. Hu, H.; Li, G.; Hu, W.; Liu, Y.; Wang, X.; Kan, Y.; Ji, M. Synthesis of Pyrrolo[2,1,5-cd]indolizines through Dehydrogenative Heck Annelation of Indolizines with Diaryl Acetylenes Using Dioxygen as an Oxidant. Org. Lett. 2015, 17, 1114-1117. [CrossRef] [PubMed]

48. Zhang, Y.; Yu, Y.; Liang, B.; Pei, Y.; Liu, X.; Yao, H.-G.; Cao, H. Synthesis of Pyrrolo[2,1,5-cd]indolizine Rings via Visible-LightInduced Intermolecular [3+2] Cycloaddition of Indolizines and Alkynes. J. Org. Chem. 2020, 85, 10719-10727. [CrossRef] [PubMed]

49. Liang, Y.; Teng, L.; Wang, Y.; He, Q.; Cao, H. Visible-Light-Induced Intermolecular [3+2] Alkenylation-Cyclization Strategy: Metal-Free Construction of Pyrrolo[2,1,5-cd]indolizine Rings. Green Chem. 2019, 21, 4025-4029. [CrossRef]

50. Yang, Y.; Chen, L.; Zhang, Z.; Zhang, Y. Palladium-Catalyzed Oxidative C-H Bond and C=C Double Bond Cleavage: C-3 Acylation of Indolizines with $\alpha, \beta$-Unsaturated Carboxylic Acids. Org. Lett. 2011, 13, 1342-1345. [CrossRef]

51. Pohjala, E.K. Indolizine derivatives. IX. Preparation of 1-acylpyrrolo[2,1,5-cd]indolizinesviacycloaddition of 3-acyloxyindolizines to active ethylenes and acetylenes. J. Heterocycl. Chem. 1978, 15, 955-960. [CrossRef]

52. Kojima, H.; Kinoshita, Y.; Matsumura, N.; Inoue, H. Reaction of tris(alkylthio)cyclopropenyl cations with 2-pyridylmagnesium bromide as a new route to indolizines. J. Heterocycl. Chem. 1991, 28, 2059-2060. [CrossRef]

53. Flitsch, W.; Heinrich, J. 1,2-Dialkoxycarbonylhydrazine derivatives of pyrroles and indolizines. A new synthesis of cycl[3.2.2]azines. Tetrahedron Lett. 1980, 21, 3673-3676. [CrossRef]

54. Yerxa, B.R.; Moore, H.W. Synthesis of indolizine-5,8-diones and [3.2.2]cyclazines. Tetrahedron Lett. 1992, 33, 7811-7814. [CrossRef]

55. Babaev, E.V. Novel Synthetic Strategies in the Chemistry of Indolizine and Its Heteroanalogs; Lambert Academic Publishing: Saarbrucken, Germany, 2016; ISBN 978-3-659-95107-7. (In Russian)

56. Kuznetsov, A.G.; Bush, A.A.; Babaev, E.V. Synthesis and reactivity of 5-Br(I)-indolizines and their parallel cross-coupling reactions. Tetrahedron 2008, 64, 749-756. [CrossRef]

57. Matsumoto, K.; Ikemi-Kono, Y.; Uchida, T.; Paquette, L.A. Synthesis and Cycloadditian Reactions of 3-Cyanoindolizines. Heterocycles 1980, 14, 103. [CrossRef]

58. Uchida, T.; Matsumoto, K. Cycloaddition reaction of 3-cyanoindolizines with dimethyl acetylenedicarboxylate-Formation of cycl[3.2.2] azines and 1:2 adducts. Chem. Lett. 1980, 9, 149-150. [CrossRef]

59. Tominaga, Y.; Shiroshita, Y.; Hosomi, A. Synthesis of 2-methylthioindolizine-3-carbonitriles using nitro ketene dithioacetal. J. Heterocycl. Chem. 1988, 25, 1745-1749. [CrossRef]

60. Matsumoto, K.; Uchida, T.; Yoshida, H.; Toda, M.; Kakehi, A. Cycloadditions of indolizine-3-carbonitriles with dimethyl acetylenedicarboxylate: Formation of [2.2.3]cyclazines and 1:2 adducts. J. Chem. Soc. Perkin Trans. 1 1992, 18, $2437-2441$. [CrossRef]

61. Matsumoto, K.; Kabuto, C.; Uchida, T.; Yoshida, H.; Ogata, T.; Iwaizumi, M. Formation of an extremely unusual 1:2 adduct from 3-cyanoindolizines and dimethyl acetylenedicarboxylate. Tetrahedron Lett. 1987, 28, 5707-5708. [CrossRef]

62. Matsumoto, K.; Uchida, T.; Sugi, T.; Kobayashi, T. Syntheses and Cycloadditions of 1,2-Aromatic Ring-fused 3-Cyanoindolizines. Heterocycles 1983, 20, 1525-1529. [CrossRef]

63. Matsumoto, K.; Uchida, T.; Aoyama, K.; Nishikawa, M.; Kuroda, T.; Okamoto, T. Synthesis and reactions of 1,2-fused 3cyanoindolizines. J. Heterocycl. Chem. 1988, 25, 1793-1801. [CrossRef]

64. Tominaga, Y.; Shiroshita, Y.; Gotou, H.; Matsuda, Y. Synthesis of Benzannelated Cycl[3.2.2]azine: Benzo[a]cycl[3.2.2]azine. Heterocycles 1986, 24, 3071-3074. [CrossRef]

65. Matsumoto, K.; Uchida, T.; Kato, T.; Toda, M.; Aoyama, K.; Konishi, H. Cycloaddition Reactions of 6-Cyanobenz[a]indolizines with Activated Alkynes. Formation of Benzo[2.2.3]- and [2.3.4]cyclazines. Heterocycles 1990, 31, 593-596. [CrossRef]

66. Mitsumori, T.; Bendikov, M.; Dautel, O.; Wudl, F.; Shioya, T.; Sato, H.; Sato, Y. Synthesis and Properties of Highly Fluorescent Indolizino[3,4,5-ab]isoindoles. J. Am. Chem. Soc. 2004, 126, 16793-16803. [CrossRef]

67. Tominaga, Y.; Gotou, H.; Oniyama, Y.; Nishimura, Y.; Matsuda, Y. Synthesis of benzannelated cycl[3.2.2]azine: Benzo[g]cycl [3.2.2]azine. Chem. Pharm. Bull. 1985, 33, 3038-3041. [CrossRef]

68. Tominaga, Y.; Shiroshita, Y.; Matsuda, Y.; Hosomi, A. The Effect of Benzannelation toward Cycl[3.2.2]azine. Synthesis and Physical Properties of Dibenzo[a,h]cycl[3.2.2]azine. Heterocycles 1987, 26, 2073-2075. [CrossRef]

69. Ohier, P.; Daïch, A.; Decroix, B. Pyrrolo[1,4]diazepines, via thermolyse of carbonylazides, and [2,2,3]cyclazines, via Diels-Alder reaction of [f]indolizines, annelated to [1]benzothiophene. Tetrahedron 1996, 52, 13547-13556. [CrossRef]

70. Godfry, J. Communications-Novel Synthesis of a 1,2,5,6-Dibenzocycl[2,2,3]azine. J. Org. Chem. 1959, 24, 581. [CrossRef]

71. Gogoi, S.; Dutta, M.; Gogoi, J.; Boruah, R.C. Microwave promoted synthesis of cycl[3.2.2]azines in water via a new threecomponent reaction. Tetrahedron Lett. 2011, 52, 813-816. [CrossRef]

72. Sheela Gopal, M.; Anitha, I. Microwave Synthesis and Characterisation of Some Cycl[2,2,3]Azine Derivatives. Int. J. Sci. Res. 2016, $5,960-963$. 
73. Komatsu, M.; Kasano, Y.; Yamaoka, S.; Minakata, S. Novel Generation of Pyridinium Ylides from N-(Silylmethyl)pyridone Analogs via 1,4-Silatropy and their 1,3-Dipolar Cycloadditions Leading to N-Heteropolycycles. Synthesis 2003, 9, $1398-1402$. [CrossRef]

74. Moise, I.-M.; Ghinet, A.; Shova, S.; Bîcu, E. Switching the reactivity of cyanomethylpyridinium salts in the 1,3-cycloaddition conditions with alkyl propiolates to cyanoindolizines or cyanoazaindolizinyl-indolizines. Tetrahedron 2020, 76, 131502-131512. [CrossRef]

75. Matsumoto, K.; Uchida, T.; Sugi, T.; Yagi, Y. Consecutive 1,3-dipolar cycloadditions of cycloimmonium ylides to benzyne: Formation of novel $18 \pi$ benzo[a]isoindolo[1,2,3-cd]indolizines. Chem. Lett. 1982, 11, 869-870. [CrossRef]

76. Matsumoto, K.; Katsura, H.; Uchida, T.; Aoyama, K.; Machiguchi, T. Formation of 1,2,3,4-dibenzocycl[2.2.3]azines by a novel consecutive 1,3-dipolar cycloaddition of pyridinium dicyanomethylides to benzyne. J. Chem. Soc. Perkin Trans. 1 1996, 21, 2599-2602. [CrossRef]

77. Matsumoto, K.; Iida, H.; Mimori, S.; Hamana, H.; Uchida, T. Nitrogen 14 NMR CP and Correlations of Oxidation Potentials of Dibenzo[a,d]cycl[2.2.3]azines with the Corresponding HOMOs: Further Evidence for Peripheral Conjugate System. Heterocycles 2007, 73, 883-889. [CrossRef]

78. Acheson, R.M.; Robinson, D.A. The formation of cycl[2,2,3]azines from pyridines and methyl propiolate. Chem. Commun. 1967, 4, 175-176. [CrossRef]

79. Acheson, R.M.; Woollard, J.M. Addition reactions of heterocyclic compounds. Part XLVI. Reactions of acetylenic esters with pyridines in the presence of proton donors, and with alkyl 3-(2-pyridyl)-trans-acrylates. J. Chem. Soc. C Org. 1971, 3296-3305. [CrossRef]

80. Pohjala, E.K. Indolizine Derivatives. V. The Perkin Reaction of 2-Pyridinecarbaldehyde. Disproportionation of 3-(2-Pyridyl)acrylic Acid. Acta Chem. Scand. B 1976, 30, 198-202. [CrossRef]

81. Pohjala, E.K. Indolizine Derivatives. VIII. 3-Acyloxyindolizines via Cyclization of Diethyl 2-Pyridylmethylenemalonate. Acta Chem. Scand. B 1977, 31, 321-324. [CrossRef]

82. Pohjala, E.K. Indolizine Derivatives. I. A Novel One-step Synthesis of Pyrrolo[2,1,5-cd]-indolizines. The Mechanism of the Acylative Cyclization of 2-Pyridinecarbaldehyde and Unsaturated Carbonyl Compounds. Acta Chem. Scand. B 1974, $28,582-583$. [CrossRef]

83. Boekelheide, V.; Miller, A. Synthesis of 2-Phenyl-1-azacyl[3.2.2]azine. J. Org. Chem. 1961, 26, 431-432. [CrossRef]

84. Boekelheide, V.; Kertelj, S.S. A Synthesis of 6-Methyl-2-phenyl-5-azacycl[3.2.2]azine and Related Compounds1,2. J. Org. Chem. 1963, 28, 3212-3213. [CrossRef]

85. Buchan, R.; Fraser, M.; Shand, C. Azaindolizines. 4. Synthesis and formylation of 8-azaindolizines. J. Org. Chem. 1977, 42, 2448-2454. [CrossRef]

86. Kurata, K.; Awaya, H.; Tominaga, Y.; Matsuda, Y.; Kobayashi, G. Synthesis of Indolizine Derivatives and Their Reactions. II. J. Pharm. Soc. Jpn. [Yakugaku Zasshi] 1978, 98, 631-635. [CrossRef] [PubMed]

87. Tominaga, Y.; Shiroshita, Y.; Kurokawa, T.; Matsuda, Y.; Hosomi, A. Synthesis of 1-azacycl[3.2.2]azine and 1-azabenzo[h]cycl[3.2.2] azine. J. Heterocycl. Chem. 1988, 25, 185-189. [CrossRef]

88. Tominaga, Y.; Shiroshita, Y.; Kawabe, M.; Goto, H.; Oniyama, Y.; Matsuda, Y. Synthesis of Benzannelated 1-Azacycl[3.2.2]azine: 1-Azabenzo[h]cycl[3.2.2]azine. Heterocycles 1985, 23, 2531-2534. [CrossRef]

89. Yamashita, M.; Horiguchi, H.; Hirano, K.; Satoh, T.; Miura, M. Fused Ring Construction around Pyrrole, Indole, and Related Compounds via Palladium-Catalyzed Oxidative Coupling with Alkynes. J. Org. Chem. 2009, 74, 7481-7488. [CrossRef] [PubMed]

90. Ghosh, M.; Naskar, A.; Mishra, S.; Hajra, A. Palladium-catalyzed dehydrogenative annulation of imidazo[1,2-a]pyridines with diarylalkynes. Tetrahedron Lett. 2015, 56, 4101-4104. [CrossRef]

91. Joshi, H.; Meena, N.; Sharma, S.; Bhatt, R.; Shinde, V.N.; Sunda, A.P.; Bhuvanesh, N.; Kumar, A. Selenium coordinated palladium(II) trans-dichloride molecular rotor as catalyst for site selective annulation of 2-arylimidazo[1,2-a]pyridines. Chem. Commun. 2020, 56, 10223-10226. [CrossRef]

92. Ghosh, S.K.; Ghosh, D.; Maitra, R.; Kuo, Y.-T.; Lee, H.M. Palladium-Catalyzed Oxidative Cyclization for the Synthesis of 2-Alkylimidazo[5,1,2-cd]indolizines. Eur. J. Org. Chem. 2016, 34, 5722-5731. [CrossRef]

93. Aginagalde, M.; Vara, Y.; Arrieta, A.; Zangi, R.; Cebolla, V.L.; Delgado-Camón, A.; Cossío, F.P. Tandem [8+2] Cycloaddition[2+6+2] Dehydrogenation Reactions Involving Imidazo[1,2-a]pyridines and Imidazo[1,2-a]pyrimidines. J. Org. Chem. 2010, 75, 2776-2784. [CrossRef]

94. Subbarayappa, A.; Semwal, R.; Joshi, A.; Kumar, R. Annulation of Imidazo[1,2-a]pyridines Under Metal-free Conditions. New J. Chem. 2020, 44, 20530-20534. [CrossRef]

95. Peng, J.; Wang, H.; Chen, C.; Huang, Z.; Yao, L.; Li, B. Palladium-Catalyzed Double C-H Arylation Reaction: Tandem Synthesis of Benzo[a]imidazo[5,1,2-cd]indolizines from Imidazo[1,2-a]pyridines and o-Dihaloarenes. Synthesis 2015, 47, 2457-2466. [CrossRef]

96. Pereira, D.E.; Leonard, N.J. A masked [ $\pi 8+\pi 2]$ cycloaddition reaction involving the 1,3,4,6-tetraazapentalene ring system. Tetrahedron Lett. 1986, 27, 4129-4132. [CrossRef]

97. Blatcher, P.; Middlemiss, D.; Murray-Rust, P.; Murray-Rust, J. 1-Phenyl-2,3-diazacycl[2,2,3]azine, a new 10- $\pi$ electron system. Involvement of 3,5-didehydroimidazo[1,5-a]pyridine? Tetrahedron Lett. 1980, 21, 4193-4196. [CrossRef]

98. Tsuchiya, T.; Kato, M.; Sashida, H. Thermal intramolecular cyclization of 2-ethynylpyridine N-ylides to indolizines and cyclazines. Chem. Pharm. Bull. 1984, 32, 4666-4669. [CrossRef] 
99. Sashida, H.; Kato, M.; Tsuchiya, T. Thermal rearrangements of cyclic amine ylides. VIII. Intramolecular cyclization of 2ethynylpyridine $\mathrm{N}$-ylides into indolizines and cycl(3.2.2)azines. Chem. Pharm. Bull. 1988, 36, 3826-3832. [CrossRef]

100. Shadrin, I.A.; Rzhevskii, S.A.; Rybakov, V.B.; Babaev, E.V. Sonogashira Reaction of the Indolizine Ring. Synthesis 2015, 47, 2961-2964. [CrossRef]

101. Pelletier, G.; Charette, A.B. Triflic Anhydride Mediated Synthesis of Imidazo[1,5-a]azines. Org. Lett. 2013, 15, $2290-2293$. [CrossRef] [PubMed]

102. Babaev, E.V.; Pasichnichenko, K.Y.; Rybakov, V.B. Heterocyclic Compounds with a Bridge Nitrogen Atom. 14. Cycloaddition of Acetylenedicarboxylic Acid Ester to 2-Chloro-N-phenacylpyridinium Ylide. Crystal Structure of Dimethyl Ester of 5-Chloro-3-(pnitrobenzoyl)indolizine-1,2-dicarboxylic Acid. Chem. Heterocycl. Compd. 2000, 36, 1192-1197. [CrossRef]

103. Rybakov, V.B.; Babaev, E.V.; Pasichnichenko, K.Y. X-ray mapping in heterocyclic design: VIII. Synthesis and X-ray diffraction study of dimethyl 3-(p-chlorobenzoyl)-5-chloroindolizine-1,2-dicarboxylate and the product of its cyclization 1,2-bis(carbomethoxy)-6chloro-3H-isoquinolino[1,2,3-d,c]indolizine-3-one. Crystallogr. Rep. 2002, 47, 622-628. [CrossRef]

104. Ghosh, S.K.; Kuo, B.-C.; Chen, H.-Y.; Li, J.-Y.; Liu, S.-D.; Lee, H.M. Double C-H Functionalization to Construct Polycyclic Heteroarenes Catalyzed by an Ionic Salt of a Pd Complex with an N-Heterocyclic Carbene Ligand. Eur. J. Org. Chem. 2015, 19, 4131-4142. [CrossRef]

105. Li, P.; Zhang, X.; Fan, X. Synthesis of Naphtho[1' $\left.2^{\prime}: 4,5\right]$ imidazo[1,2-a]pyridines and Imidazo[5,1,2-cd]indolizines through PdCatalyzed Cycloaromatization of 2-Phenylimidazo[1,2-a]pyridines with Alkynes. J. Org. Chem. 2015, 80, 7508-7518. [CrossRef]

106. Simonyan, V.V.; Zinin, A.I.; Babaev, E.V.; Jug, K. Mechanism of cycloaddition to indolizines. J. Phys. Org. Chem. 1998, 11, 201-208. [CrossRef]

107. Babaev, E.V.; Simonyan, V.V.; Pasichnichenko, K.Y.; Nosova, V.M.; Kisin, A.V.; Jug, K. Quantum Chemical Analysis and Experimental Study of the Cycloaddition Reaction between Aminoacetylenes and 6-Nitroindolizines. NMR and ab Initio Evidence for the [4+2] Adduct Formation. J. Org. Chem. 1999, 64, 9057-9062. [CrossRef]

108. Yamashita, Y.; Suzuki, D.; Masumura, M. Addition Reaction of Indolizine Derivatives with Dimethyl Acetylenedicarboxylate. Heterocycles 1984, 22, 705-708. [CrossRef]

109. Siberdt, F.; Nasielski, J. Indolizines 3. The synthesis of 2-vinylindolizines and their reaction with dienophiles. Bull. Soc. Chim. Belg. 1997, 106, 85-96.

110. Zinin, A.I.; Babaev, E.V. Unexpected reaction of 6-nitroindolizine with dimethyl acetylenedicarboxylate. Russ. Chem. Bull. 1997, 46, 609-610. [CrossRef]

111. Blake, A.J.; Dick, J.W.; Leaver, D.; Strachan, P. Heterocyclic Compounds with Bridgehead Nitrogen Atoms. Part 11. I Formation of Azocino[2,1,8-cd]pyrrolizines in the Reactions of lndolizines with Dimethyl Acetylenedicarboxylate. J. Chem. Soc. Perkin Trans. 1 1991, 2991-2997. [CrossRef]

112. Ikeda, S.; Kajigaeshi, S.; Kanemasa, S. Peripheral conjugate systems 1. Cycloaddition of indolizine to electron-deficient olefin. Chem. Lett. 1976, 5, 367-372. [CrossRef]

113. Kajigaeshi, S.; Mori, S.; Fujisaki, S.; Kanemasa, S. exo-Selective Peripheral Cycloaddition Reactions of Pyrido[2,1-a]isoindole. Bull. Chem. Soc. Jpn. 1985, 58, 3547-3551. [CrossRef]

114. Dick, J.W.; Gibson, W.K.; Leaver, D.; Roff, J.E. Heterocyclic compounds with bridgehead nitrogen atoms. Part 9. Synthesis in the pyrrolo[2,1,5-de]quinolizine ([2.3.3]cyclazine) series starting from indolizines. J. Chem. Soc. Perkin Trans. 1 1981, $3150-3157$. [CrossRef]

115. Batroff, V.; Flitsch, W. Ein neuer Zugang zu [2.3.4]Cyclazinen. Liebigs Ann. Chem. 1987, 7, 621-628. [CrossRef]

116. Jasiński, R.; Dresler, E. On the Question of Zwitterionic Intermediates in the [3+2] Cycloaddition Reactions: A Critical Review. Organics 2020, 1, 49-69. [CrossRef] 